\title{
TOWARD A GENERAL DESCRIPTION OF TWO-BODY HADRON REACTIONS
}

\author{
B.J. HARTLEY \\ Laboratoire de Physique Theorique et Particules Flementaires, Orsay, France \\ G.L. KANE * \\ Physics Department, University of Michigan, Ann Arbor, Michigan
}

Received 23 rebruary 1973

Abstract: We present a model for describing two-body high-energy hadron reactions at energies above the resonance region. Although no real theory yet exists, we can give a procedure for obtaining a (possibly correct) description of the amplitudes for any two-body reaction (what. ever the spins involved, including real and inlaginary parts of amplitudes, any helicity-flip $\pi$-exchange as well as vector and tensor exchanges, etc.). The most important new physical effect involved is using the appropriate $t$-dependent phase for the vacuum exchange amplitude (the pomeron), both in elastic scattering and in describing absorption effects. The pomeron is not a Regge pole.

The model is very simple to formulate and has a reasonable physical interpretation in terms of important unitarity effects and absorption. It allows us to understand the partial successes and the inadequacies of previous approaches such as the dual absorptive model, the strong absorption model, the view that some amplitudes have Regge-pole behavior while others do not, etc. In the present paper we analyze all the $0^{-\frac{1}{2}}{ }^{+} \rightarrow 0^{-} \frac{1}{2}^{+}$reactions, $\mathrm{np} \rightarrow \mathrm{pn}$, and $\bar{p} p \rightarrow \bar{n} n$, cross sections, polarizations and amplitudes; these include all the important kinds of helicity amplitudes for any two-body reactions. Apart from a few places the results are very good; we argue that the few places where there may be some difficulty are due to effects we have left out rather than to the structure of the model.

Al though the input reggeon exchanges are not all exchange degenerate, the phases of the output amplitudes after absorption show a remarkable and unexpected resemblance to what one would expect from exchange degenerate poles, apart from the necessity of having a zero structure related to important absorption corrections.

A number of related results, some of which are considered puzzles, are discussed (total cross-section differences for $\pi N$ and $K N$, the increase in $\sigma_{\mathrm{T}^{(}}\left(\mathrm{K}^{+} \mathrm{p}\right)$, crossovers, shrinkage, real parts of elastic amplitudes, polarization single and double zeros, line reversed reactions, $p p$ scattering at the ISR, etc.) and some predictions are given.

* Research supported in part by the US Atomic Energy Commission. Much of this work was done while the author was a J.S. Guggenheim Fellow visiting the Rutherford Laboratory. 


\section{Introduction}

There is no need to emphasize how far we are from possessing a theory of hadron interactions at the present time. For those who are not content to wait for some inspired individual to produce a theory, a promising path is to slowly increase the extent to which we can understand the experimental data on hadron interactions in terms of reasonable physical ideas. In this paper we try to pursue this approach for two-body, high-energy, hadron interactions.

In the following we conjecture, al though we cannot yet derive, a procedure for obtaining a description of all helicity amplitudes and their phases for two-body reactions in terms of a few parameters. All the parameters have (we believe) a simple physical interpretation and their values are generally a priori approximately known. Fven though the formulation of the model is extremely simple, leaving out many effects which will ultimately have to be included, (such as $t$-channel unitarity, lowlying exchanges, etc.), the model appears to be able to give a unified description of all elastic and single-particle exchange reactions and a good description of most existing high-energy data. It accounts for many observed effects, such as the different behaviors of $\Delta \sigma_{T}(\pi \mathrm{p})$ and $\Delta \sigma_{\Upsilon}(\mathrm{Kp})$, the rise of $\sigma_{\Gamma}\left(\mathrm{K}^{+} \mathrm{p}\right)$, signs of forward real parts, polarization zeros, polarization in $n p \rightarrow p n$ and $\bar{p} p \rightarrow n n$ (the latter was calculated before there was data), the lack of a large negative polarization in $\pi^{-} p \rightarrow \pi^{0} n$, the ISR small $t \mathrm{~d} \sigma(\mathrm{pp}) / \mathrm{d} t$, and others, in a simple way.

In addition, it is possible to see why various previous approaches $[1,2]$ (such as the weak or strong absorption models, the dual absorptive model, the view that flip amplitudes were Regge-pole behaved, exchange degeneracy for flip amplitudes, etc.) were sometimes successful, sometimes inadequate or inapplicable.

Almost all two-body reactions can in practice be described in terms of four kinds of s-channel helicity amplitudes. We have included examples of all four amplitudes in our analysis, so no new kinds of amplitudes will be encountered in extending our model to most reactions. Thus we are entitled to be very optimistic about further applications of our model. We have tried to state clearly how the model can be applied to any two-body reaction by any high-energy physicist interested in analyzing data or obtaining predictions. Although difficulties in describing data may well show up in the future, at the moment it seems to us that the biggest problem is providing a theoretical basis for our conjectures on the structure of the pomeron amplitude and the absorption procedure.

\section{Model}

We will state the model as a definite procedure here and discuss the motivation and implications of each step in the next section.

Consider any two-body process

$a+b \rightarrow c+d$, 
where the particles have helicities $\lambda_{\mathrm{a}}, \lambda_{\mathrm{b}}, \lambda_{\mathrm{c}}, \lambda_{\mathrm{d}}$. Define the helicity-flip quantum numbers $n$ and $x$ by putting

$$
m_{\mathrm{a}}=\lambda_{\mathrm{c}}-\lambda_{\mathrm{a}}, \quad m_{\mathrm{b}}=\lambda_{\mathrm{d}}-\lambda_{\mathrm{b}}, \quad n=\left|m_{\mathrm{b}}-m_{\mathrm{a}}\right|, \quad n+x=\left|m_{\mathrm{b}}\right|+\left|m_{\mathrm{a}}\right| .
$$

Then calculate the $s$-channel helicity amplitudes with the following assumptions.

(A) For each particle that can be exchanged the amplitude for a definite parity reggeon is

$$
R_{\lambda_{\mathrm{c}} \lambda_{\mathrm{d}} ; \lambda_{\mathrm{d}} \lambda_{\mathrm{b}}}=-(-t)^{\frac{1}{2}(n+x)} \gamma_{\mathrm{ca}} \gamma_{\mathrm{bd}} \Gamma\left(\frac{1}{2}(J-\alpha)\right)\left(\frac{s}{s_{\mathrm{o}}} \mathrm{e}^{-\frac{1}{2} i \pi}\right)^{\alpha} \mathrm{e}^{-\frac{1}{2} i \pi J},
$$

where $\alpha=\alpha(t)$ is the reggeon trajectory (in practice assumed linear in the region of interest), $J$ is the spin of the lowest physical particle on the trajectory ( 0 for $\pi$, 1 for $\rho$ and $\omega, 2$ for $\mathrm{f}$ and $\mathrm{A}_{2}, \frac{1}{2}$ for $\mathrm{N}, \frac{3}{2}$ for $\Delta$, etc.), and $\gamma_{\mathrm{ca}}^{\mathrm{R}}, \gamma_{\mathrm{db}}^{\mathrm{R}}$ are factorized residues (assumed to be constants). Relations among residues due to symmetries such as parity invariance can be obtained by the method of ref. [3].

(B) For elastic scattering, the pomeron amplitude for $n=0$ (non-flip amplitude) has the form

$$
P(s, t)=-i s\left[A \mathrm{e}^{B t}+A_{\mathrm{o}} \mathrm{e}^{B_{\mathrm{o}} t} J_{\mathrm{o}}\left(R_{\mathrm{o}} \sqrt{-t} \sqrt{\ln s-\frac{1}{2} i \pi}\right)\right] .
$$

We arbitrarily assume the energy scale is $\mathrm{GeV}^{2}$ for the pomeron.

(C) For quasielastic processes (e.g., diffraction dissociation), s-channel helicity amplitudes with net helicity flip $n$ have a pomeron contribution of the form

$$
D_{n}(s, t)=-i s \mathrm{e}^{D t} J_{n}\left(R_{\mathrm{o}} \sqrt{-t} \sqrt{\ln s-\frac{1}{2} i \pi}\right) .
$$

(D) As in most conventional absorption models, we calculate the full $s$-channel helicity amplitude for the process $a+b \rightarrow c+d$ from

$$
M_{\lambda_{c} \lambda_{\mathrm{d}} ; \lambda_{\mathrm{a}} \lambda_{\mathrm{b}}}(s, t)=2 q^{2} \int_{\mathrm{o}}^{\infty} b \mathrm{~d} b R_{\lambda_{\mathrm{c}} \lambda_{\mathrm{d}} ; \lambda_{\mathrm{a}} \lambda_{\mathrm{b}}}(s, b) S_{\mathrm{cff}}(s, b) J_{n}(b \sqrt{-t}),
$$

or equivalently

$$
M_{\lambda_{c} \lambda_{d} ; \lambda_{\mathrm{a}} \lambda_{\mathrm{b}}}(s, t)=\sum_{J}(2 j+1) R_{\lambda_{\mathrm{c}} \lambda_{d} ; \lambda_{\mathrm{a}} \lambda_{\mathrm{b}}}^{j}(s) S_{\mathrm{eff}}^{j}(s) d_{\lambda_{\mathrm{a}}-\lambda_{\mathrm{b}}, \lambda_{c}-\lambda_{d}}(\cos \theta),
$$

where $R(s, b)$ and $R^{j}(s)$ are given by the usual transforms

$$
R_{\lambda_{\mathrm{c}} \lambda_{\mathrm{d}} ; \lambda_{\mathrm{a}} \lambda_{\mathrm{b}}}(s, b)=\frac{1}{2 q^{2}} \int_{\mathrm{o}}^{\infty} \sqrt{-t} \mathrm{~d} \sqrt{t} R_{\lambda_{\mathrm{c}} \lambda_{\mathrm{d}} ; \lambda_{\mathrm{a}} \lambda_{\mathrm{b}}}(s, t) J_{n}(b \sqrt{-t}),
$$




$$
R_{\lambda_{\mathrm{c}} \lambda_{\mathrm{d}} ; \lambda_{\mathrm{a}} \lambda_{\mathrm{b}}}^{j}(s)=\frac{1}{2} \int \mathrm{d} z R_{\lambda_{\mathrm{c}} \lambda_{\mathrm{d}} ; \lambda_{\mathrm{a}} \lambda_{\mathrm{b}}}(s, t) d_{\lambda_{\mathrm{a}}-\lambda_{\mathrm{b}}, \lambda_{\mathrm{c}}-\lambda_{\mathrm{d}}}^{j}(z)
$$

with similar definitions for any impact parameter transform or partial wave expansion. This is formally equivalent to the usual form of the absorption model, but depends crucially on the specification of $S_{\text {eff. We assume for a given reaction that all }}$ exchanges are absorbed with the same $S_{\text {eff }}$, and that $S_{\text {eff }}$ is independent of helicities.

(E) With the definition

$$
S_{\mathrm{eff}}(s, b)=1-\frac{i q}{4 \pi W} M_{\mathrm{eff}}(s, b),
$$

we assume that

$$
M_{\mathrm{eff}}(s, b)=P(s, b)+D_{\mathrm{o}}(s, b)
$$

Here, $D_{\mathrm{o}}$ represents the contribution from the sum over intermediate states other than the elastic one. We assume that it can be well approximated by the non-flip quasielastic contribution $D_{0}$, i.e., that it arises mainly from the edge in impact parameter and that it does not fall in strength with energy except from shrinkage. Thus the effective strength of the absorption is given by all of the elastic plus diffractive production intermediate states which can couple to the pomeron. No reggeon contributions are included and the diffractive production contribution is only assumed to be important in $S_{\text {eff }}$ for helicity non-flip (diffractive production contributions which flip helicities will ultimately have to be included in $S_{\text {eff }}$, but they will only contribute a correction to most effects).

With the above procedure, by adding together the contributions of the pomeron and the appropriatc reggeons, one can construct any s-channel helicity amplitude for any two-body process.

\section{Motivation}

Our basic philosophy is that unitarity effects are of overriding importance in determining the structure of hadron interactions and that the unitarity effects can be approximately taken in to account at high energies by treating hadron interactions as largely absorptive. The Born term is defined by a reggeon exchange, of definite party, whose intrinsic quantum numbers and symmetry properties characterize the exchange, but whose $s$ - and $t$-dependence and size is strongly modified by the unitarity effects. Our Born term should be thought of as the part of the amplitude which actually has the particle poles with factorizable residues and the phase appropriate to a power-law energy dependence $s^{\alpha}$.

(A) The various pieces of the reggeon contribution can be understood as follows. Most are described in ref. [4]. 
The residues $\gamma_{\mathrm{ca}}$ and $\gamma_{\mathrm{db}}$ are assumed to go with factorized pole vertices, and to be constant for our purpose: we can use them repeatedly from one place to another. The overall plus or minus sign is to allow consistent definition of the factorized residues.

The function $\Gamma\left(\frac{1}{2}(j-\alpha)\right)$ contains the particle poles [4]. It is a simple form which has particle poles at every other integer on the Regge trajectory, beginning at the lowest allowed state whose spin is $J$, and no unphysical poles. In the absence of arguments which input more physics, it is the sensible propagator to take. It is interesting and important to note that because of the $J$ in the $\Gamma$-function the reggeon remembers the range of force; because spin and mass increase together, the force gets less peripheral as the mass of the lowest particle which can be exchanged increases, just as would be expected from a naive point of view. Thus there is considerable physics in this choice of propagator form (see fig. 1).

Because all bound states and resonances in quantum mechanics and nuclear physics lie on Regge trajectories, and because we believe particle exchanges are in the same category, we assume that pole amplitudes have a Regge power law $s^{\alpha}$ where $\alpha$ is a function of $t$. General analyticity and crossing arguments then require that the amplitude have a phase e ${ }^{-\frac{1}{2} i \pi \alpha}$. For fermions there are some subtleties about phases, and the appropriate pole amplitudes can be found in ref. [5].

Note that we are assuming that the amplitude with a definite parity reggeon exchange has no zeros away from $t=0$. Our attitude is that zeros are surely introduced into the full amplitude by the absorption correction (see below), and we will not introduce any zeros directly into the pole unless we find a place where the experimental data explicitly requires one. So far this has not occurred.

(B) Here we discuss the interpretation and motivation for our pomeron amplitude [6]. We assume that the pomeron structure is almost completely determined by $s$ channel unitarity effects, and that there are two different sorts of contributions. In the $s$-channel unitarity sum there is an important part due to complicated structureless intermediate states, e.g., pionization. This contribution behaves as a featureless term falling off smoothly in $t$ and we assume it can be approximated by a constant $x \mathrm{e}^{B t}$ for small $t$.

On the other hand, we also believe there is an important contribution from peripheral (perhaps two-body-like) terms in the unitarity sum, which is equivalent to saying they arise in some sense from the edge of the proton, a peripheral structure. From the impact parameter point of view, we assume that a peripheral structure or an edge contribution gives rise to a smoothly falling term times the Bessel function $J_{0}$ of $R \sqrt{-t}$. This gives the second term in the pomeron. Although we speak of the pomeron, it is clear that we always mean the full vacuum contribution to the elastic amplitude. The pomeron is not a Regge pole.

There are two different arguments which lead us to believe that we should choose the energy dependence of the radius to be $R^{2}$ proportional to $\ln s$. The first is just that the two-body origin of this term suggests that it will remember its Regge behavior with $R^{2}$ behaving like $\alpha^{\prime} \ln s$. From a different point of view, the increase of 
the radius with increasing energy arises because the multiplicity increases with increasing energy [7]. Then there are more virtual particles around during collisions at higher energy and there is a greater probability of a collision at a large impact parameter than there was at lower energy at that impact parameter. This approach gives $R^{2}$ growing like the multiplicity, or In $s$.

To find the phase of the pomeron we give two different points of view. The first, for the theorist, is just that we want to have a pomeron amplitude of even signature only, and we can insure this by insisting that it be a function only of the variable ( $s \mathrm{e}^{--\frac{1}{2} i \pi}$ ). This is the form that appears in eq. (3).

A completely different and naive point of view which gives a similar result is as follows: Just as the edge in impact parameter space gives rise to a contribution to the imaginary part, so it will give a contribution to the real part. But we still want the pomeron to be purely imaginary at $t=0$. Therefore the real part must have a second contribution presumably of shorter range. To have the total area in impact parameter be zero, so that the pomeron is pure imaginary at $t=0$, these two contributions give the difference of two $J_{0}$ 's. That difference is essentially the derivative of $J_{0}$, which is $J_{1}$ evaluated at a point between the two. Thus if the imaginary part of the pomeron is $J_{0}\left(R_{0} \sqrt{\cdots t} \sqrt{\ln s}\right)$, the real part of the pomeron will go like $\sqrt{\cdots t} J_{1}\left(R_{0} \sqrt{-t} \sqrt{\ln s}\right)$. Physically one could even imagine the radii in the real and imaginary parts being different, if the edge contribution to the real part is really at the edge and the shorter-range contribution is further in; then the radius in the real part will be a little bit smaller than that in the imaginary part.

The similarity of the two forms obtained is clear when we note that at higher energies

$$
J_{\mathrm{o}}\left(R_{\mathrm{o}} \sqrt{-t} \sqrt{\ln s-\frac{1}{2} i \pi}\right)=J_{\mathrm{o}}\left(R_{\mathrm{o}} \sqrt{-t \ln s}\right)+\frac{i \pi R_{\mathrm{o}} \sqrt{-t}}{4 \sqrt{\ln s}} J_{1}\left(R_{\mathrm{o}} \sqrt{-t \ln s}\right) .
$$

Thus the naive impact parameter argument and the analy ticity argument agree on the physics.

In a theory which gave rise to such a pomeron, the even signature could come about in a complicated way; the procedure above to get even signature is of course not unique. Thus, al though the naive argument about the real part in impact parameter space does not obviously give us just an even signature contribution (al though it is close to it), it is possible that it represents the physical situation more faithfully.

A different way to say it is that we might not have the $s$-dependence of $\operatorname{Im}$ (pom) exactly right. It could be that we go wrong in a way which has little effect on the actual energy dependence of the amplitudes but which has a big effect on the phase through the phase-energy relation. Thus determining the real part by a separate physical argument may be a good procedure. Even so, in practice the simplicity and economy that arise from having exactly an even signature pomeron probably justify using it in fitting data.

Evidence for our pomeron. There are currently several pieces of evidence (in addition to its success in meson-baryon elastic scattering in the present paper) which can be interpreted as support for our elastic amplitude. These are 
(i) Several studies, based on quite different assumptions, have given, at $6 \mathrm{GeV} / c$ in $\pi \mathrm{N}$ scattering, the absolute phase of the isoscalar exchange helicity non-flip amplitude, including the present analysis. They differ quantitatively, but all have found a real part which looks qualitatively like ours and has a zero at some $-t$ value before $1 \mathrm{GeV}^{2}$, and an imaginary part which decreases monotonically and has no zero. The $\pi \mathrm{N}$ absolute phase has been fixed by constraints from finite-energy sum rules [8], $t$-channel unitarity [9], dispersion relations $[10]$ and the absorption model $[11,12]$.

(ii) Quite independently of these analyses, analysis of nucleon-deuteron scattering, particularly including deuteron polarization has suggested [13] the need for an elastic nucleon-nucleon $t$-dependent phase consistent with that of our pomeron.

(iii) As we have often emphasized in this paper, a pomeron with our phase makes the absorption model work well for many non-elastic processes. If the physical assumptions underlying the absorption model are valid, this is also support for our pomeron amplitude.

(iv) The main qualitative features of the smaller- $t$ ISR data [33] on pp elastic scattering, i.e., the increase in slope as $-t$ decreases below about $0.15 \mathrm{GeV}^{2}$, and the more rapid shrinkage in the $-t$ region below about $0.15 \mathrm{GeV}^{2}$ than above it, are naturally explained [6] by our pomeron.

An additional, possibly non-trivial, point is that we currently know of no phenomenological evidence against a pomeron amplitude with $s$ - and $t$-dependence such as ours.

(C) The arguments for the quasielastic or diffraction dissociation processes are essentially the same as for the pomeron. But in this case because there is no elastic coherence, the central piece is presumably missing or very small. Similarly, because these contributions are smaller, the contributions with non-zero helicity flip will in general be important for the quasielastic processes, so that one has to use the full set of amplitudes. In ref. [14] a detailed discussion is given to motivate these forms and to suggest that existing data is consistent with the amplitudes obtained from them. For our purpose here we only need the diffractive production with net helicity-flip zero proportional to the Bessel function $J_{\mathrm{o}}$ to use in $S_{\mathrm{eff}}$. The main property that matters is that the diffractive production arise mainly from the cdge and is more peripheral than the elastic pomeron.

(D) We have nothing to add here to the formal expression for the absorption correction that has been used by many workers in the past; it is the interpretation of $S_{\text {eff }}$ that we wish [4] to modify. From the high-energy point of view, the partial wave expansion or impact parameter expansion points of view are equivalent and will give the same results for any reaction. They can differ considerably in the continuation to lower energies.

The derivation of the standard absorption correction is not especially compelling. One of the more remarkable aspects is that different workers have always arrived at exactly the same answer - even the Russian analyses [15], based on physics which is not simply connected to absorption ideas, obtained this answer. It is tempting to consider the possibility that in some sense the amplitude form given in eq. (5) is exact 
rather than a crude approximation, and that the theoretical problem is to properly interpret the sum over intermediate states which is involved in obtaining $S_{\text {eff }}$ from $M^{\mathrm{el}}$ and other contributions.

(E) Our basic understanding of how to choose $S_{\mathrm{eff}}$ is at a rather low level. The form we actually used is determined by a combination of theory, simplicity and phenomenology. Any model for $S_{\text {eff }}$, of course, will include the pomeron contribution in $M_{\text {eff }}$, and this is indeed the most significant contribution with many implications for the structure of the absorbed amplitude. We believe, in addition, that one should include an important contribution $[4,15]$ from intermediate states in addition to the clastic ones. And we assume that this can be represented by the diffractive term included. Since the diffractive term arises mainly from the edge in impact parameter, the shape of $M_{\text {eff }}$ and $M_{\text {elastic }}$ in impact parameter are not the same [4].

We also assume that no reggeons contribute to $M_{\text {eff. }}$. That is, we do not use the full elastic amplitude in constructing $M_{\mathrm{eff}}$ but only the pomeron part of elastic scattering. This means that there are no Regge-Regge cuts in the absorbed reggeon $\mathrm{ex}$ change. We do this for two reasons: the first is that Worden [16] has recently shown that many reactions (e.g., $\pi^{-} \mathrm{p} \rightarrow \pi^{\mathrm{O}} \mathrm{n}$ ) have no Regge-Regge cuts in exchange degenerate theory. Although we have no particular confidence in the detailed selection rules of such theories our final amplitudes have many exchange degenerate properties and we would not be surprised if approximate selection rules of this sort were to hold. The second reason is the great simplicity which such a procedure affords us. If there were reggeons in $M_{\mathrm{eff}}$ we would have to construct them by an iterative procedure, first absorbing with pomeron plus diffractive to get the absorbed reggeon, then putting the absorbed reggeon in $M_{\text {eff }}$, etc. As it is now, we can simply construct $M_{\text {eff }}$ once and for all for each process, and absorb all reggeons in the same way. This is a very important simplification for practical work.

There is still considerable opportunity for obtainirg a deeper theoretical insight in to the structure of $M_{\text {eff }}$.

General structure of scattering amplitudes. In many aspects our model is close to a simple quantum mechanical view of hadrons as strongly absorbing matter, or black discs. The essential modernizations of this view are mainly due to particles behaving as reggeons and to the $t$-dependent phase of the pomeron. In this section we give a qualitative discussion of the general structure of the amplitudes, particularly those aspects which deviate from naive absorption models.

Concerning the reggeons an important role is played for us by the fact that the propagator remembers the range of the force. This is illustrated in fig. 1, which shows $\Gamma(z)$ for $z=\frac{1}{2}(J-\alpha)$ in the range it covers when $0 \leqslant-t \leqslant 1$. One can see clearly how exchanged trajectories give more peripheral contributions as the lowest-spin physical contribution allowed on the trajectory goes toward lighter mass. Such an effect occurs in a different way in models with signature zeros, where the peripherality is essentially determined by the zero structure.

Next consider the pomeron. At sinall $t$ the $J_{\text {o }}$ piece is important and gives a contribution whose slope is fixed by the requirement that $J_{0}$ has a diffractive zero near 


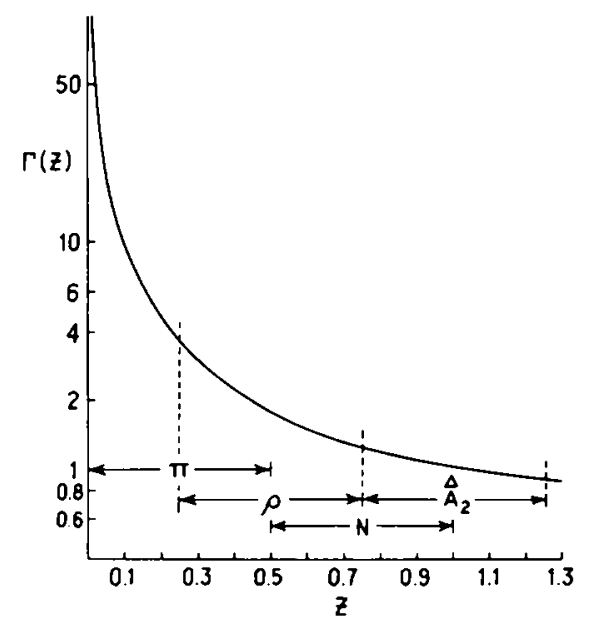

Fig. 1. A graph of $\Gamma(z)$ versus $z$ showing the region of $z$ where $t$ varies from 0 to $1 \mathrm{GeV}^{2}$. Note, for example, how the $\rho$ is more peripheral than the $A_{2}$, as seen by the sharper forward peak. For a given exchange $z=\frac{1}{2}(J \cdot \alpha(t))$, wherc $J$ is the spin of the least massive physical particle on the trajectory.

$\cdots t=0.2 \mathrm{GcV}^{2}$. Since the radius grows as $\sqrt{\ln s}$ this contribution shrinks as the energy increases. It is responsible for the behavior of the pp cross section at the ISR for $-t \lesssim 0.1 \mathrm{GeV}^{2}$ which can thus be thought of as a diffractive effect. For larger $-t$ the $J_{0}$ changes sign and interferes destructively with the central contribution, giving a flattening of the imaginary part, though not a minimum since the exponentials due to the spread-out nature of the edge and center provide damping. The real part of the pomeron, which is present for theoretical consistency, is mainly observable through its effect on elastic polarizations in the $5-15 \mathrm{GeV} / c$ range and through its effects on absorption.

We can understand the effect of the real part of the pomeron on the absorbed amplitudes as follows. It is worth some trouble to do this since in our model this is the main physical effect accounting for the structure of polarizations and for the remarkable exchange degenerate behavior of the absorbed amplitudes.

Consider vector exchange (c.g., $\rho$ or $\omega$ ). Then the reggeon amplitude is proportional to

$$
R \sim i \mathrm{e}^{-\frac{1}{2} i \pi \alpha}
$$

so for small $t$

$$
\begin{aligned}
& \operatorname{Re} R \sim+\sin \frac{1}{2} \pi \alpha, \\
& \operatorname{Im} R \sim+\cos \frac{1}{2} \pi \alpha .
\end{aligned}
$$

Then the full amplitude is given by ( $M$ is the full absorbed amplitude, $R$ the reggeon, and $P$ the pomeron) 


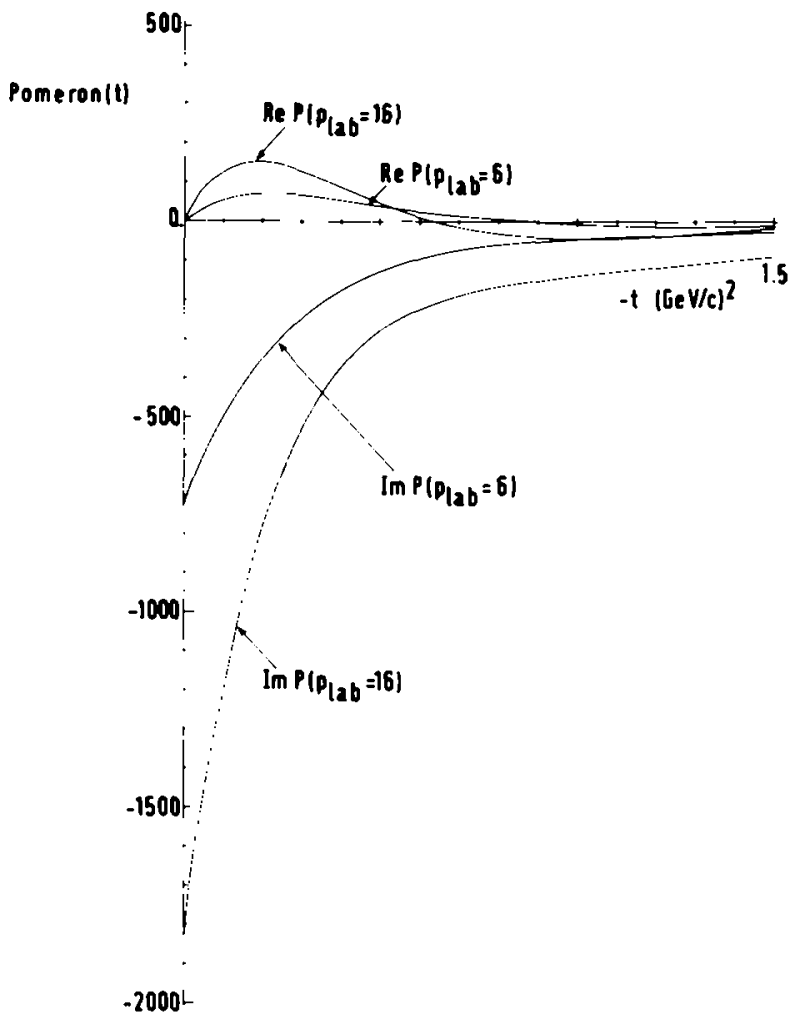

Fig. 2. The real and imaginary parts of the pomeron amplitude are shown (they have opposite sign), at two energies to illustrate the energy dependence, for the global fit.

$$
M(s, t) \sim R(s, t)-i \int \mathrm{d} t_{1} \mathrm{~d} t_{2} K\left(t, t_{1}, t_{2}, s\right) R\left(s, t_{1}\right) P\left(s, t_{2}\right),
$$

where $K$ is a known real positive kinematic function; this form is equivalent to the forms (which are more convenient for numerical work) given above. It says one has a kind of double scattering. Then

$$
\begin{aligned}
& \operatorname{Re} M \sim \operatorname{Re} R+\int(\operatorname{Re} R \operatorname{Im} P+\operatorname{Im} R \operatorname{Re} P), \\
& \operatorname{Im} M \sim \operatorname{Im} R+\int(\operatorname{Im} R \operatorname{Im} P-\operatorname{Re} P \operatorname{Re} R) .
\end{aligned}
$$

For the important region of integration $\operatorname{Re} R, \operatorname{Im} R$ and $\operatorname{Re} P$ are positive, while $\operatorname{Im} P$ is negative (with our conventions, where $\operatorname{Im} P(s, t=0)=$ is $\sigma_{\mathrm{T}}$ ). The dominant contribution at small $t$ in each is the old contribution with $\operatorname{Im} P$. For the imaginary part the old contribution, $\operatorname{Im} P \operatorname{Im} R$, is enhanced by $\operatorname{Re} P \operatorname{Re} R$. Thus the $\operatorname{Im} M$ for vector exchange is absorbed more strongly due to the $\operatorname{Re} P$.

On the other hand, for $\operatorname{Re} M$ the new contribution $\operatorname{Re} P \operatorname{Im} R$ tends to cancel the 


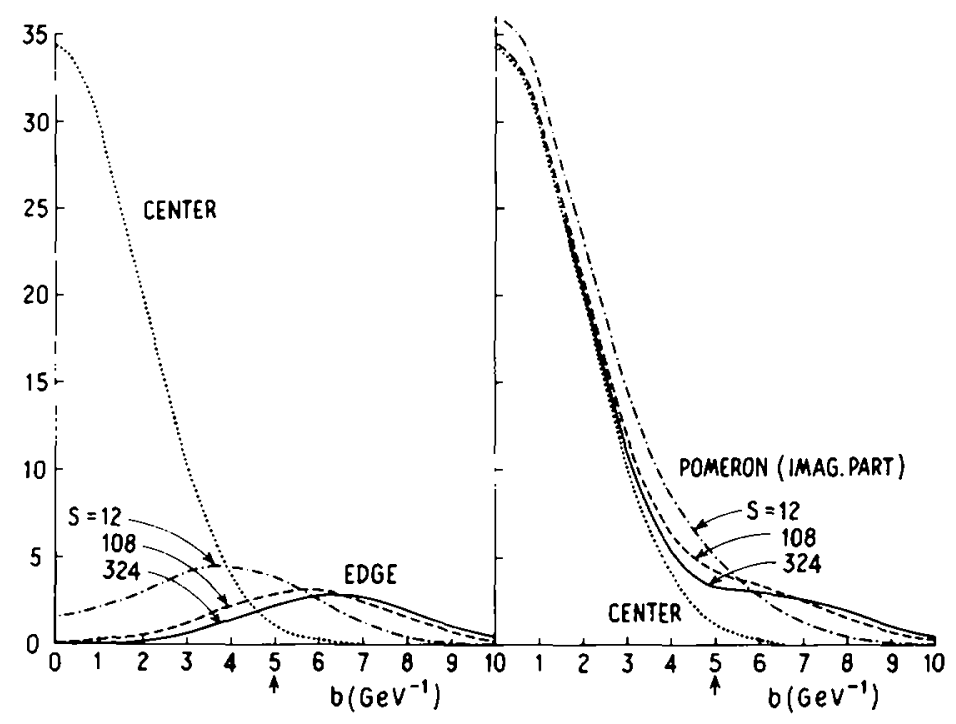

Fig. 3. (a) The pieces of the imaginary part of the pomeron are shown in impact parameter to illustrate the shapes and sizes of the central and edge contributions and the energy dependence of the edge contribution; $s$ is given in $\mathrm{GeV}^{2}$. (b) The central contribution is shown again and the full pomeron given by central plus edge contributions. The arrow shows one fermi. (These curves are for a Gaussian central region rather than the black disc mentioned in the comments section.)

old absorbing piece $\operatorname{Im} P \operatorname{Re} R$, so the $\operatorname{Re} M$ is absorbed less. Further, as $-t$ increases the integral in $\operatorname{Re} M$ has a zero because of the term with $\operatorname{Re} P$ so that for $t$-values larger than about $-t \approx \frac{1}{2} \mathrm{GeV}^{2}$ one has a positive contribution to $R e R$ rather than a negative one. This would be obvious if all terms were evaluated at the same $t$; because of the integration it is subtle, but it must happen because $\operatorname{Im} P(t) \operatorname{Re} R(t)$ has a zero (becoming positive) near $-t=0.5$ while $\operatorname{Im} R(t) \operatorname{Re} P(t)$ is positive for all $-t \lesssim 1$.

Thus it is clear physically how the presence of $\operatorname{Re} P$ rotates the absorbed amplitude. Quite remarkably, the final result (e.g., in fig. 5) contains an imaginary part for the full $\rho$-amplitude which behaves as in the naive strong absorption model, with a very nice diffractive zero, while the real part of the full $\rho$-amplitude has been rotated up to have a double-zero structure resembling that of a $\rho$ Regge pole with a signature zero when $\alpha_{\rho}=0$ (although the large secondary maximum of the pure pole after the double zero is generally not present). It is basically the real part of the pomeron which produces in our model this pattern so of ten noticed in the past as characteristic of the data, with the imaginary part of the vector exchange strongly absorbed while the real part is not far from the exchange degenerate pole amplitude.

For tensor exchange (e.g., $f, A_{2}$ ) one can see what happens from the structure of the pole term. First, the signature is opposite so relative to vector there is a factor of $i$; thus $\operatorname{Re} T \sim-\operatorname{Im} V$ and $\operatorname{Im} T \sim \operatorname{Re} V$. In addition, the tensor exchanges are less pe. ripheral so the zeros are at larger values of $-t$. Altogether, we expect the real parts 

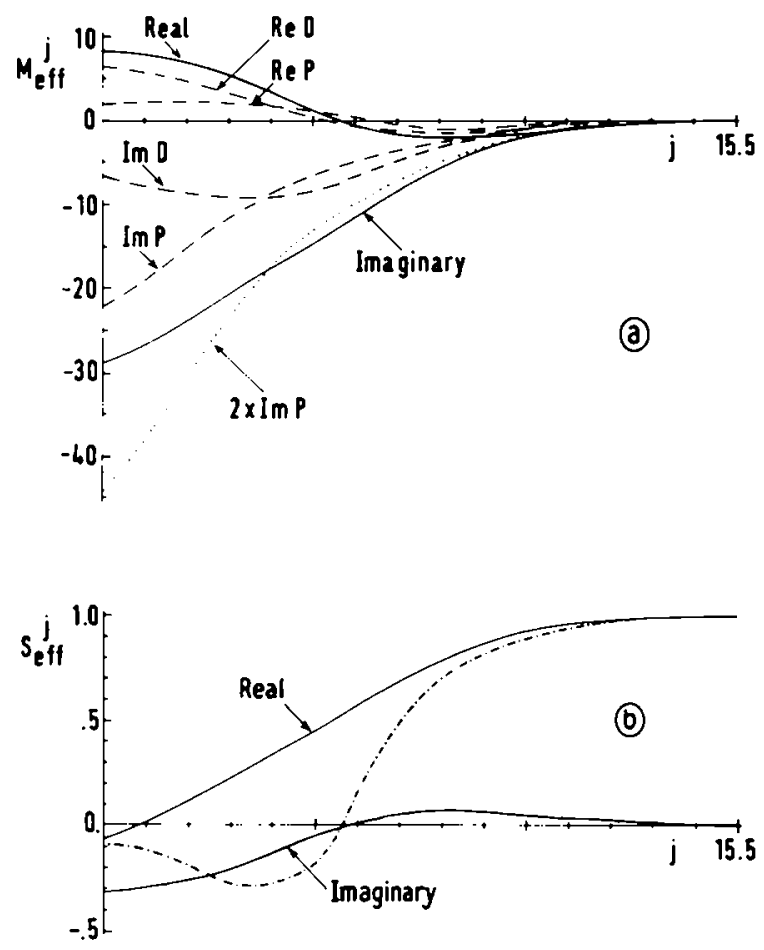

Fig. 4. (a) The global fit pomeron and effective diffractive dissociation contributions to $M_{\text {eff }}^{j}$ at $6 \mathrm{GeV} / c$ for $\pi \mathrm{N} \rightarrow \pi \mathrm{N}$. The normalization is as in the text. (b) The resulting $S_{\text {eff }}^{j}$ is also shown. Note the different shapes of $\operatorname{Im} M_{\text {eff }}$ and $2 \operatorname{Im} P$ (which is similar to the old $\lambda \operatorname{Im} M_{++}$ (elastic)). This shows how much more our hadrons behave as absorbing discs than the ones of the older strong absorption model. Also shown in part (b) is a (preliminary) result from ref. [18] for $\operatorname{Re} S_{\text {eff }}$ for NN scattering, to show that the main difference coming from the larger $\sigma_{\mathrm{T}}$ of $\mathrm{NN}$ will be to give a blacker disc, rather than extra absorption at the center (dash-dotted line).

of the tensor amplitudes to exhibit the peripheral zeros but at larger $-t$ values, while the imaginary parts of the tensor amplitudes will be rotated toward the double. zero structure as the real parts of the vector amplitudes were. However, since tensor exchanges are so short range they depend considerably on the details of the absorption of the low partial waves. Considerable caution should be used in interpreting results that are sensitive to that.

Next let us look at the structure of the amplitude in the approximate situation where the input pole is simply taken as $s^{\alpha(t)} \mathrm{e}^{-\frac{1}{2} i \pi \alpha(t)}$. (This approximation is only used in this section.) This will illustrate nicely some qualitative features, particularly the energy dependence and shrinkage behavior.

For illustrative purposes here, then, we write the pole as

$$
R(s, t) \approx s \mathrm{e}^{-\frac{1}{2} i \pi \alpha(t)}=a \mathrm{e}^{\alpha^{\prime}\left(\ln s-\frac{1}{2} i \pi\right) t},
$$


where $a=s^{\alpha_{0}} \mathrm{e}^{-\frac{1}{2} i \pi \alpha_{0}}$. Let us calculate the absorption correction with

$$
M_{\mathrm{eff}}=-i s\left[A \mathrm{e}^{B t}+A_{\mathrm{o}} \mathrm{e}^{B_{0} t} J_{\mathrm{o}}(R \Delta)\right]+s A_{1} \mathrm{e}^{B_{1} t} J_{1}\left(R^{\prime} \Delta\right),
$$

where the coefficients $A, A_{0}$ and $A_{1}$ are positive and

$$
\begin{aligned}
& R^{2}=R_{\mathrm{o}}^{2} \ln s, \\
& \Delta^{2}=-t .
\end{aligned}
$$

Using the two identities

$$
\begin{aligned}
& \int \mathrm{d} \Delta^{2} \mathrm{e}^{-b \Delta^{2}} J_{\mathrm{o}}(r \Delta)=\left(\mathrm{e}^{-r^{2} / 4 b}\right) / b, \\
& \int \mathrm{d} \Delta^{2} \mathrm{e}^{-b \Delta^{2}} J_{\mathrm{o}}(r \Delta) J_{\mathrm{o}}(R \Delta)=\frac{1}{b} I_{\mathrm{o}}\left(\frac{r R}{2 b}\right) \mathrm{e}^{-\left(r^{2}+R^{2}\right) / 4 b},
\end{aligned}
$$

one can then calculate analy tically all of the Hankel transforms and the absorbed amplitude. The result is

$$
M(s, t)=R(s, t)-C(s, t),
$$

where $M$ is the full amplitude, $R$ is the reggeon and $C$ is the absorption correction,

$$
\begin{aligned}
& C(s, t)=\frac{A a}{8 \pi(B+b)} \mathrm{e}^{b B t /(b+B)} \\
& \times\left[1+\frac{A_{0}}{A} \frac{B+b}{B_{\mathrm{o}}+b} \mathrm{e}^{\left[b B_{0} /\left(b+B_{0}\right)\right][b B /(b+B)] t} \mathrm{e}^{-R^{2} / 4\left(b+B_{0}\right)} J_{\mathrm{o}}\left(\frac{b R \Delta}{b+B_{\mathrm{o}}}\right)\right] \\
& \quad+\left(\text { due to } \operatorname{Re} M_{\text {eff }}\right) .
\end{aligned}
$$

For our purposes now we ignore the effect of $\operatorname{Re} M_{\mathrm{eff}}$, whose effect is probably more easily understood in terms of the arguments we gave above. First note the 1 in the bracket and the standard coefficient $\exp [b B /(b+B)] t$; these come from the central term and are exactly of the form one obtained in the past for absorption with a fixed pure imaginary pomeron. It is very important to understand, however, that here the strength of this term is even less than that of the elastic contribution since in the pomeron one makes up $\sigma_{\mathrm{T}}$ from the coefficients of both the central and edge terms in about an equal mixture.

Now recall that under our assumptions the various quantitics have an energy dependence

$$
b \sim \ln s, \quad R^{2} \sim \ln s, \quad B, B_{\mathrm{o}}, B_{1} \sim \text { constant } .
$$




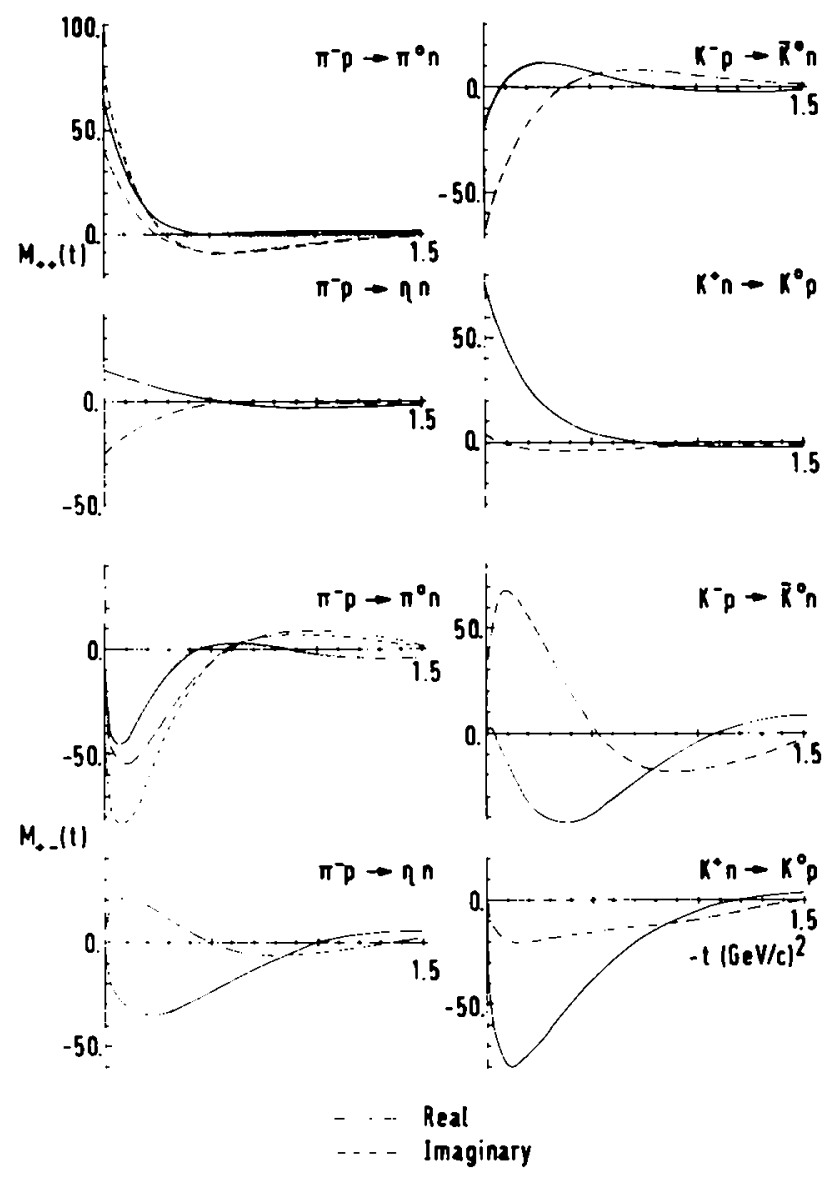

Fig. 5. Global fit non-flip and flip final amplitudes for $\rho, A_{2}$ and $\pm \rho+A_{2}$ exchanges in $\pi N$ and $\mathrm{KN}$ charge exchange at $6 \mathrm{GeV} / c$. The real and imaginary parts of the $\rho$-amplitudes at $16 \mathrm{GeV} / \mathrm{c}$ are shown as dotted and dot-dashed lines respectively. Note the remarkable resemblance to exchange degenerate phases, with the $K^{+} n \rightarrow K^{0} p$ amplitudes mainly real, the $K^{-} p \rightarrow \bar{K}^{\circ} n$ amplitudes showing a rotating phase, and the $\eta n$ flip amplitude, where absorption is smaller because of the helicity flip, having the right sort of zero structure.

The most important thing to note is then the factor

$$
\exp \left(-R^{2} / 4\left(B_{\mathrm{o}}+b\right)\right) \approx \exp \left(-R_{\mathrm{o}}^{2} \ln s / 4\left(p+\alpha^{\prime} \ln s\right)\right)
$$

(with $p \gtrsim 2 \mathrm{GeV}^{-2}$ in most models where it includes $B_{\mathrm{o}}$ and a contribution from $t$-dependence in the pole which we have ignored here). If there were not a $\ln s$ in the denominator above this factor would give a power-law decrease of the effect on absorption of the edge contribution! In fact, it begins as a power-law decrease at lower 


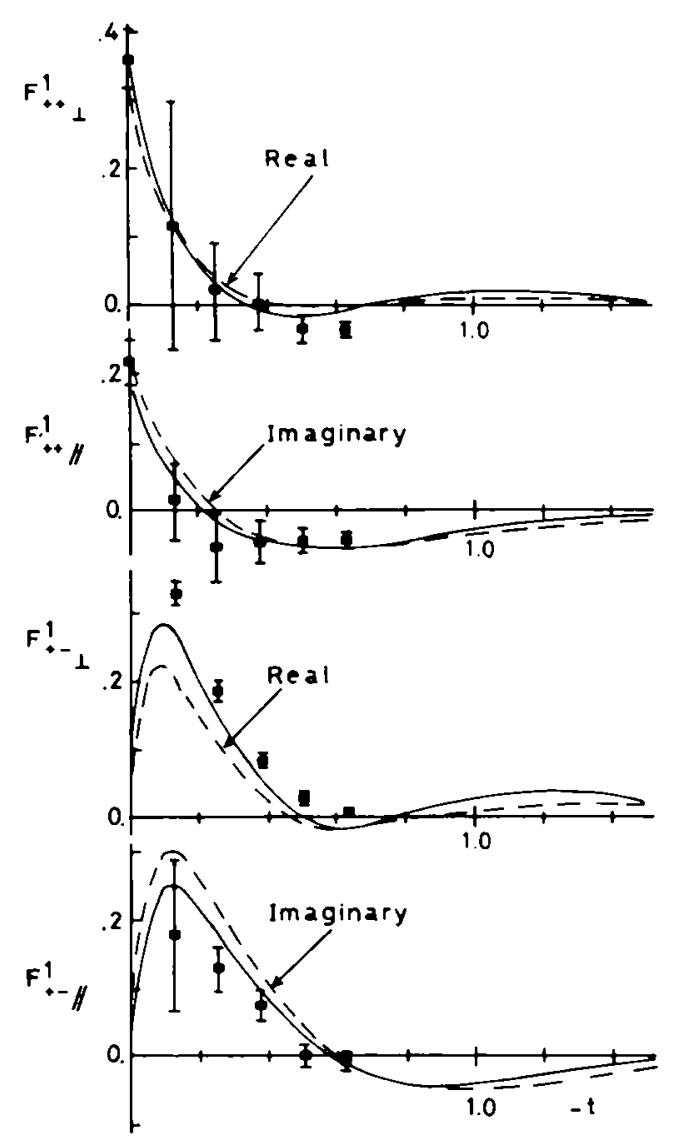

Fig. 6. The global fit isovector amplitudes in $\pi \mathrm{N}$ scattering at $6 \mathrm{GeV} / c$ compared with the results of Halzen and Michael from ref. [19]. (We use their notation in this figure.)

$s$ and goes to a constant limit (apart from the shrinkage of $J_{0}$ away from $t=0$ ) as $\ln s \gg p$. Thus the effective strength of the absorption decreases rapidly with energy at a few $\mathrm{GeV} / \mathrm{c}$ and settles down bey ond about $20 \mathrm{GcV} / \mathrm{c}$ to cssentially a constant strength. Whether this final value is larger than or less than the absorption that would result from elastic absorption cannot yet be determined a priori; for our results we have asymptotically an effective absorption strength of only about $90 \%$ of that we would obtain from using $M_{\text {eff }}=-i s \sigma_{\mathrm{T}} \mathrm{e}^{B t}$, while at $6 \mathrm{GeV} / c$ the effective absorption is about $25 \%$ greater than we would get from $M_{\mathrm{eff}}=-i s \sigma_{\mathrm{T}} \mathrm{e}^{B t}$; these numbers are calculated just by comparing the size of $C(s, t=0)$ at various energies.

The most important insight the above analysis gives us is an understanding of the way in which the present model solves one of the main problems of all previous models with absorption or cuts, the shrinkage problem (this has been most empha- 

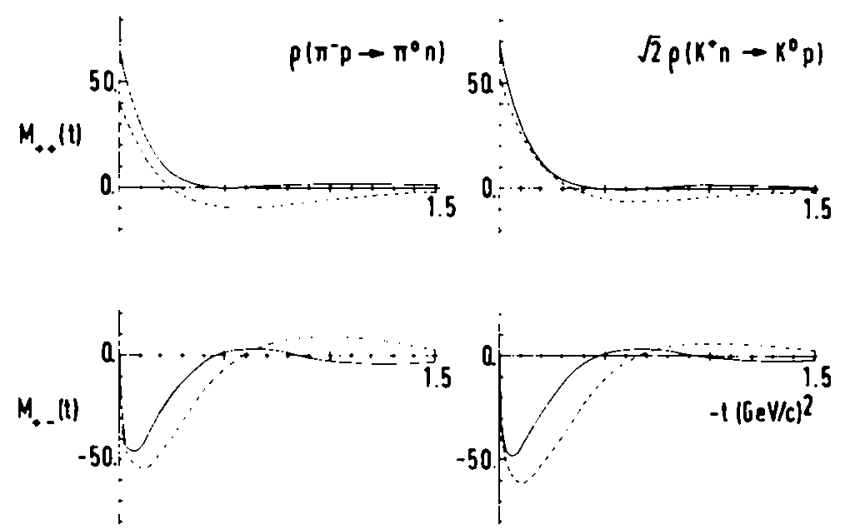

\section{Real \\ Imaginary}

Fig. 7. The global fit $\rho$-amplitude for $\pi^{-\mu} p \rightarrow \pi^{o} n$ and $K^{+} n \rightarrow K^{o} p$, to show the effect of having different absorption because $\sigma_{\mathrm{T}}(\pi \mathrm{N})>\sigma_{\mathrm{T}}(\mathrm{KN})$. Note the shift in the place where $\mathrm{Im} M_{++}=0$, which is reflected in the $\mathrm{KN}$ crossover zero being further out than the $\pi \mathrm{N}$ crossover (assuming $\rho$ and $\omega$ are otherwise the same).

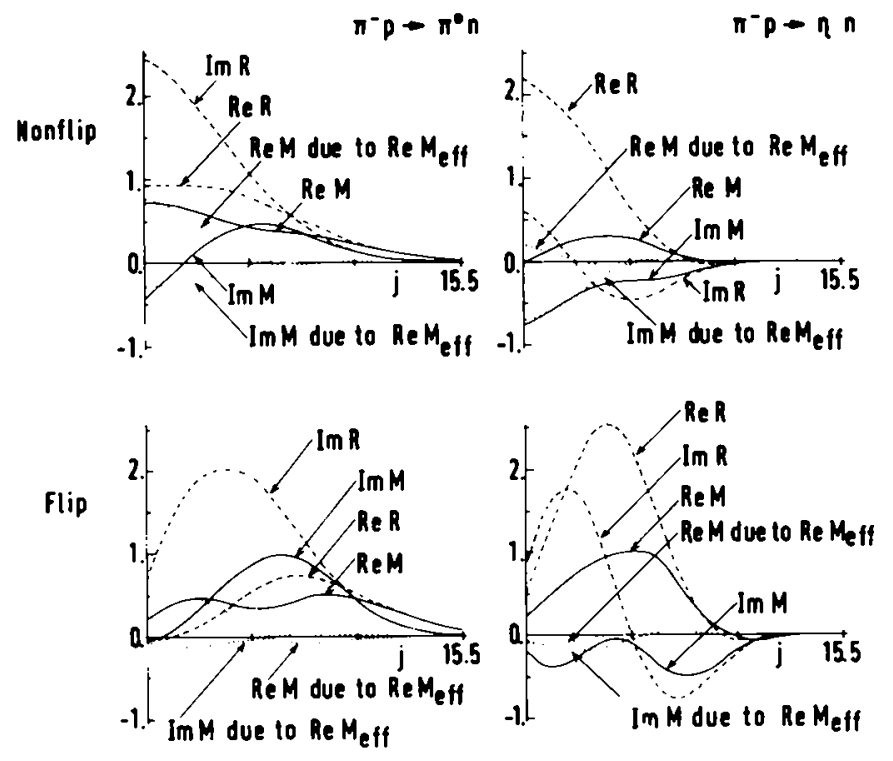

Fig. 8. Global fit partial wave projections (equivalent to impact parameter) of the $\rho$ and $A_{2}$ ex. change amplitudes in $\pi^{-} \mathrm{p} \rightarrow \pi^{\circ} \mathrm{n}$ and $\pi^{-} \mathrm{p} \rightarrow \eta \mathrm{n}$ at $6 \mathrm{GeV} / \mathrm{c}$. One $\mathrm{fm}$ is at $j \approx 7.5$. Note for the non-flip amplitude that $\operatorname{Im} M$ for vector exchange and $\operatorname{Re} M$ for tensor exchange show simple peripheral behavior. 

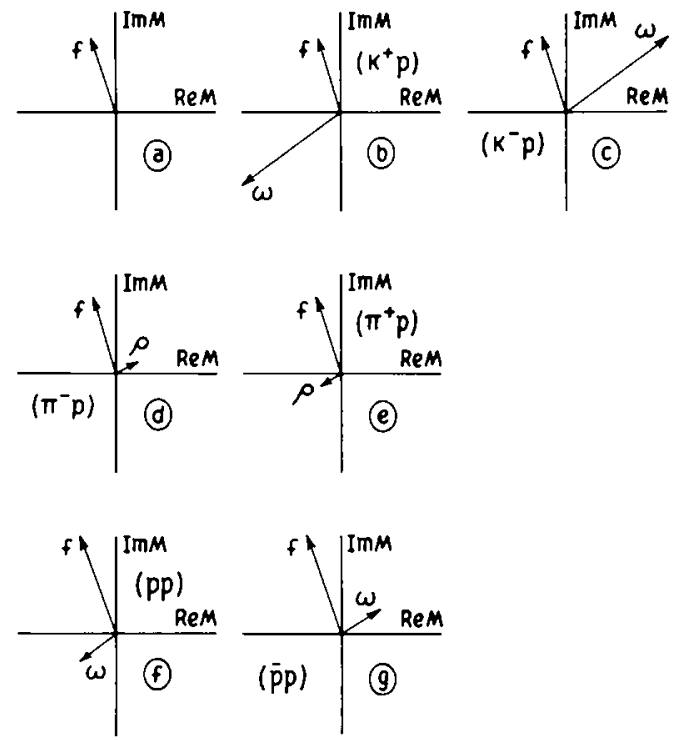

Fig. 9. Argand diagrams showing the size and phases of various contributions to various elastic process, to help understand the energy dependence of $\sigma \Upsilon$ and the forward real parts of these processes. The implications are described in the text. Part (b) shows why $\sigma_{\mathrm{T}}\left(\mathrm{K}^{+} \mathrm{p}\right.$ ) rises due to reggeons $\omega$ and $f$ dying out as $s$ increases.

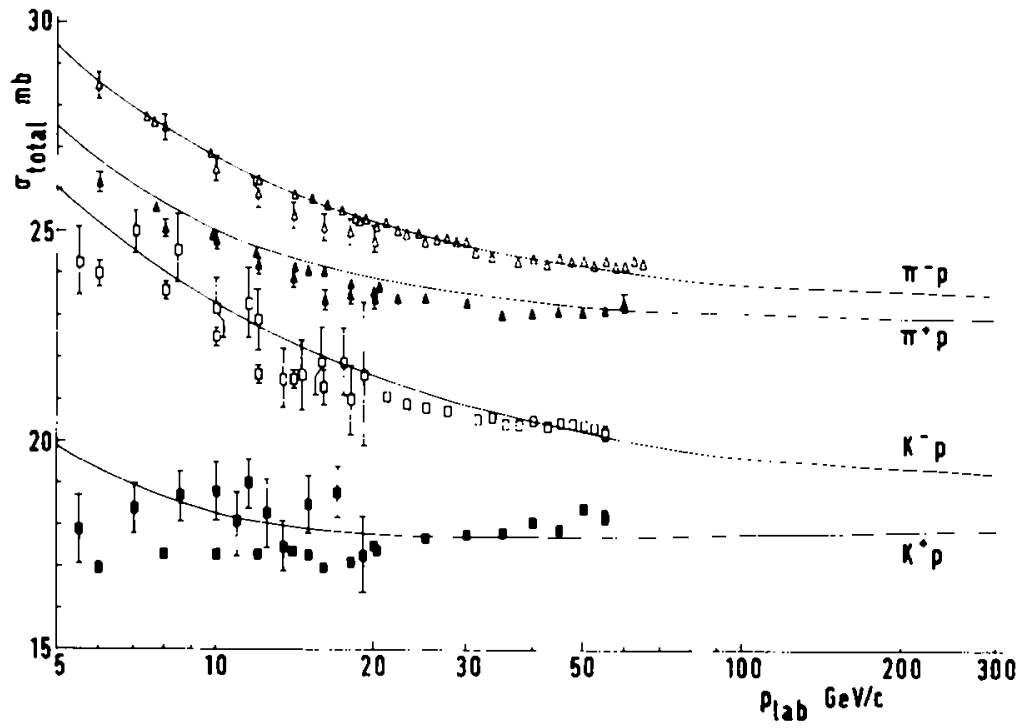

Fig. 10. Global fit total cross sections for $\pi^{ \pm} p$ and $K^{ \pm} p$ scattering. Data from ref. [36]. See text for detailed discussion of $\Delta \sigma_{\mathrm{T}}$ behavior and tise of $\sigma_{\mathrm{T}}\left(\mathrm{K}^{+} \mathrm{p}\right)$; the latter is entircly due to reggeons going away with energy in our model and could account for the observed behavior. 


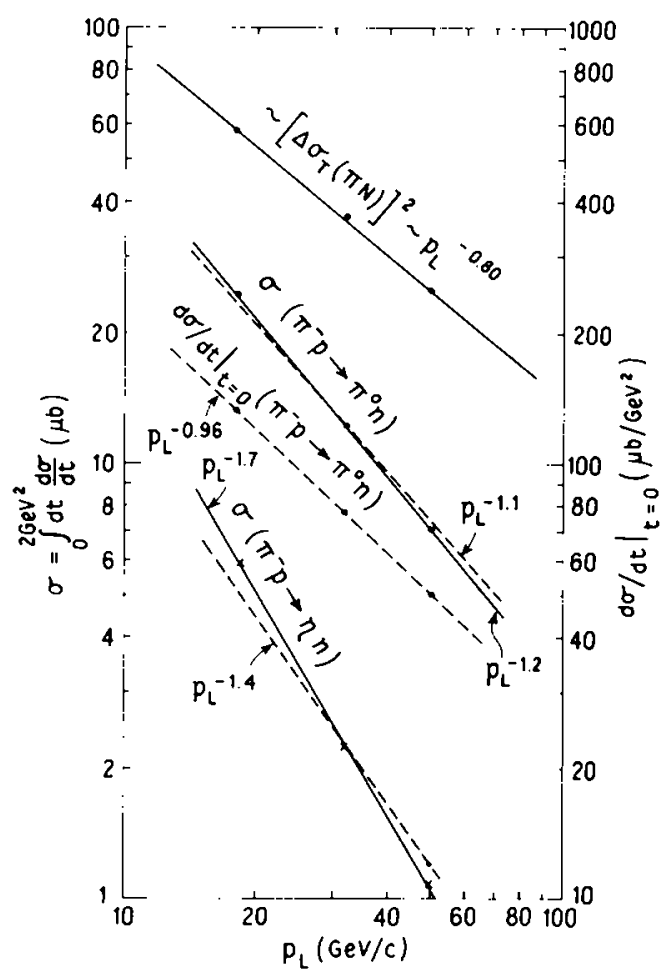

Fig. 11. Theoretical energy dependence of several quantities compared for the global fit. The left-hand scale shows integrated differential cross section in $\mu \mathrm{b}$ and the right hand scale $\mathrm{d} \sigma / \mathrm{d} t$ at $t=0$ in $\mu \mathrm{b} / \mathrm{GeV}^{2}$. For $\pi^{-} \mathrm{p} \rightarrow \pi^{\circ} \mathrm{n}$ one can compare $\left[\Delta \sigma_{\mathrm{T}}(\pi \mathrm{N})\right]^{2}$ which is proportional to the square of $\operatorname{Im} M\left(\pi^{-} p \rightarrow \pi^{\circ} \mathrm{n}\right)$ at $t=0$, do/d $t$ at $t=0$ which is proportional to $(\operatorname{Im} M)^{2}+(\operatorname{Re} M)^{2}$ at $t=0$, and the integrated cross section. These would all have the same energy dependence in a pole model and behave as shown in our absorption model. See discussion in text for detailed comparison with data. For the integrated cross sections for $\pi^{-} p \rightarrow \pi^{\circ} n$ and $\pi^{-} p \rightarrow \eta n$ the dashed lines show the behavior of the experimental results from Serpukov.

sized in ref. [17]). Essentially this problem is that in the past the cuts, with a flatter slope $(b B /(b+B)<b)$, have dominated at larger $t$ and sometimes (as in $\pi^{-} \mathrm{p} \rightarrow \pi^{\circ} \mathrm{n}$ ) they give too little shrinkage. Here we see that the cut strength decreases with energy so that the cut has a larger effective shrinkage at energies below $10-15 \mathrm{GeV} / c$. Asymptotically the naive cut slope does dominate, but there is no longer any disagreement between experiment and theory concerning shrinkage at a few $\mathrm{GeV} / c$. When one amplitude dominates in this energy range one will now see shrinkage; when several are important they will usually still add up over a short energy range in a way to hide most of the shrinkage of the individual amplitudes.

In this section we have seen qualitatively how the present model solves the two major problems of previous absorption models for two-body reactions, the phase 


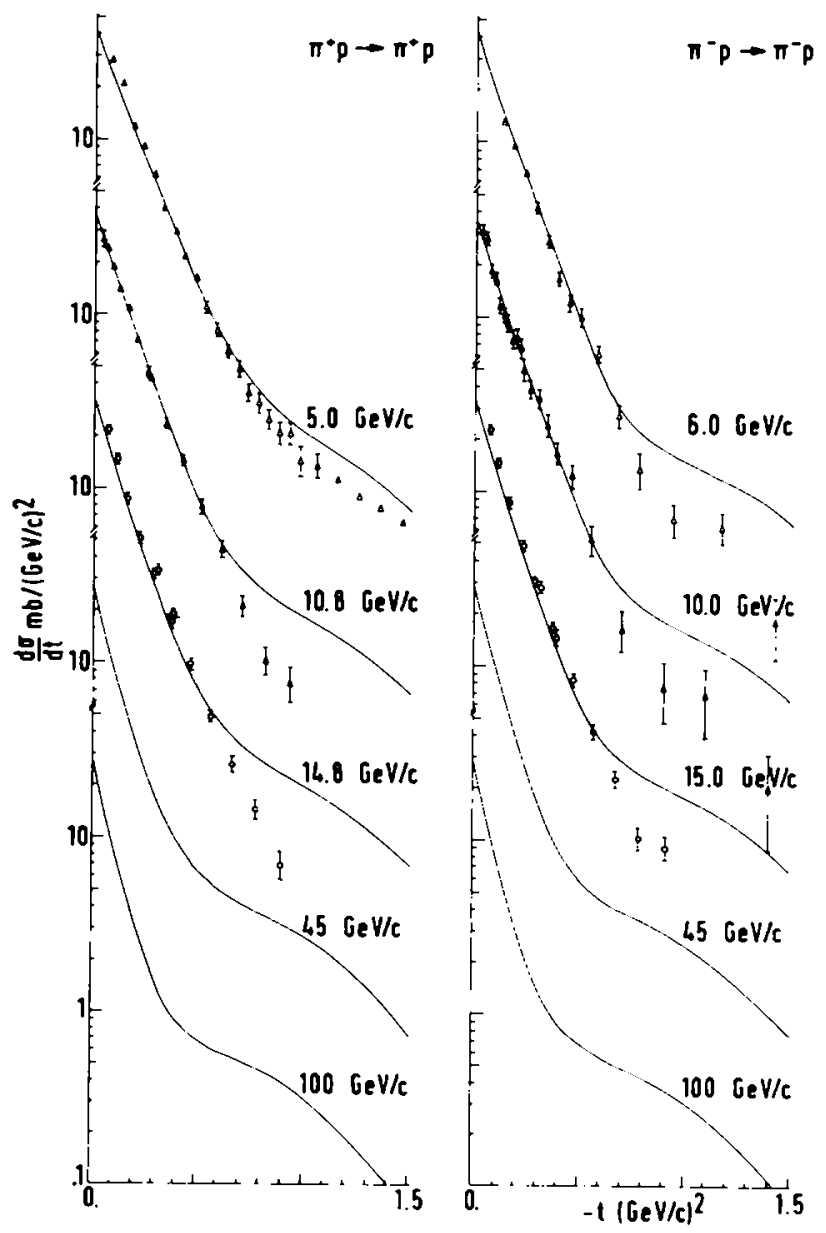

Fig. 12. Global fit differential cross sections for $\pi^{ \pm} p \rightarrow \pi^{ \pm} p$. Data from ref. [37].

problem (e.g., that real and imaginary parts of vector exchange amplitudes did not appear to originate from the same mechanism), and the shrinkage problem (that absorbed amplitudes continued to shrink at low energies in a way similar to the poles). Now we discuss the description of the experimental data.

\section{Physical interpretation of parameters}

Here we discuss how one should go about comparing our model with experimental data and how one should interpret the quantities in the amplitudes which are not predetermined. 

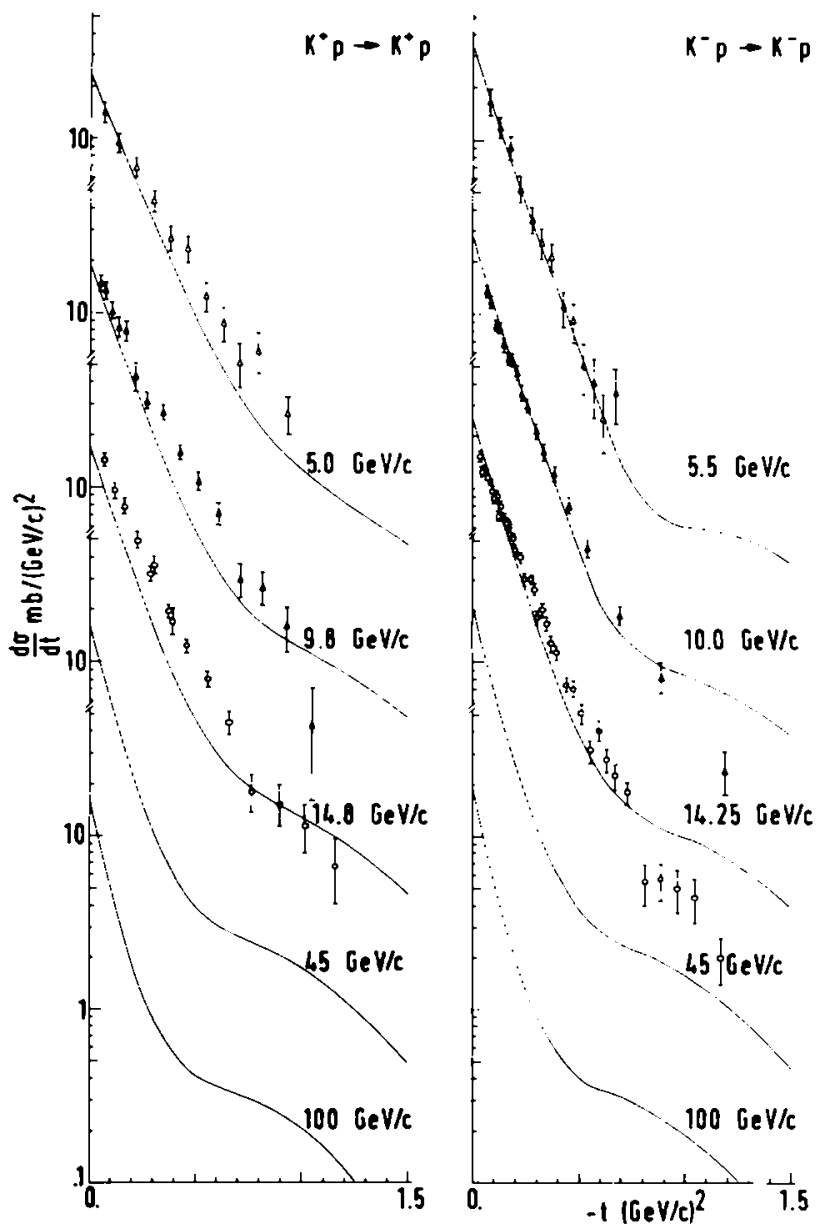

Fig. 13. The $\mathrm{K}^{ \pm} \mathrm{p}$ elastic differential cross sections calculated from global fit. Data from ref. [38].

For the reggeon the situation before we look at any data is as always - each reg. geon has a trajectory, each amplitude has a strength, and there are the energy scale factors $s_{\mathrm{o}}$. Although this seems like a lot of parameters for one exchange, it is not if that exchange is simultaneously considered in several reactions, and if $\alpha^{\prime}$ and/or $s_{\mathrm{o}}$ are fixed ahead of time. We assume all trajectories have unit slope, and all have the same $s_{\mathrm{o}}$ (except possibly for the $\mathrm{A}_{2}$ ).

For the pomeron we have to determine $A, A_{\mathrm{o}}, B, B_{\mathrm{o}}, R$. Consider a given elastic reaction. We will see that the latter three of these have a clear physical interpretation which constrains them. If the effective fall-off in momentum transfer is as $\mathrm{e}^{B t}$, it is as $e^{-b^{2} / 4 B}$ in impact parameter. One fm corresponds to $5 \mathrm{GeV}^{-1}$. Thus if the center has a width of $0.6 \mathrm{fm}=3 \mathrm{GeV}^{-1}$ (choosing this number since it must be significantly 


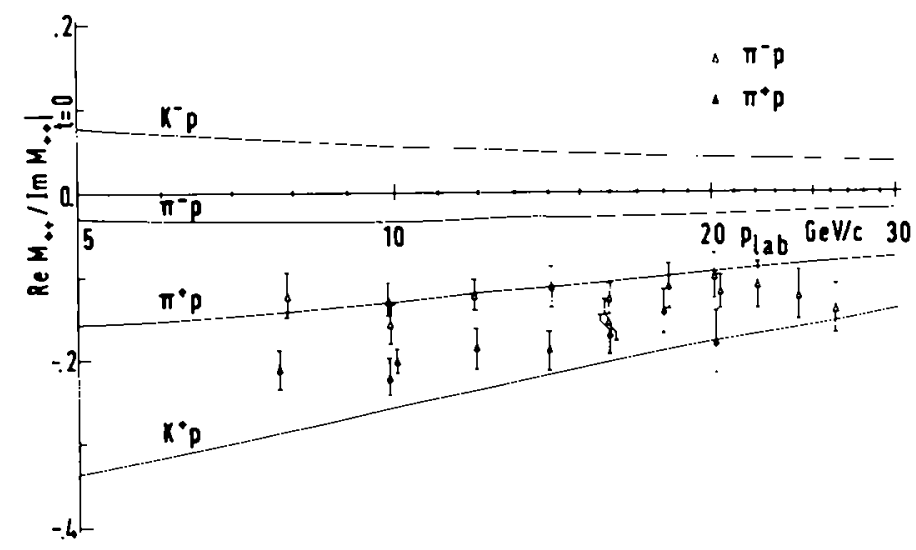

Fig. 14. The global fit ratio of ( $\left.\operatorname{Re} M_{++} / \operatorname{lm} M_{++}\right)_{t=0}$ for $\pi^{ \pm} \mathrm{p}$ and $\mathrm{K}^{ \pm} \mathrm{p}$ elastic scat tering. The data are from ref. [39]. The signs of all four follow from general arguments given in the text.

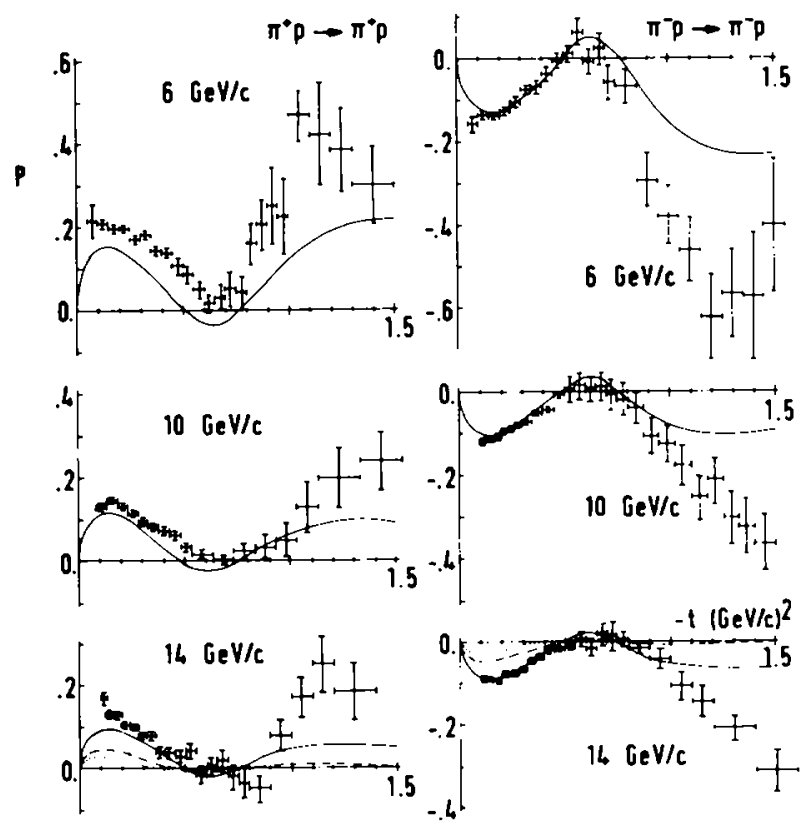

Iig. 15. Global fit polarizations for $\pi^{ \pm} p \rightarrow \pi^{ \pm}$. Data from ref. [40]. The predicted polarizations at $45 \mathrm{GeV} / c$ and $100 \mathrm{GeV} / c$ are shown by dashed and dotted lines, respectively. The isoscalarflip amplitude has been assumed to be zero. 

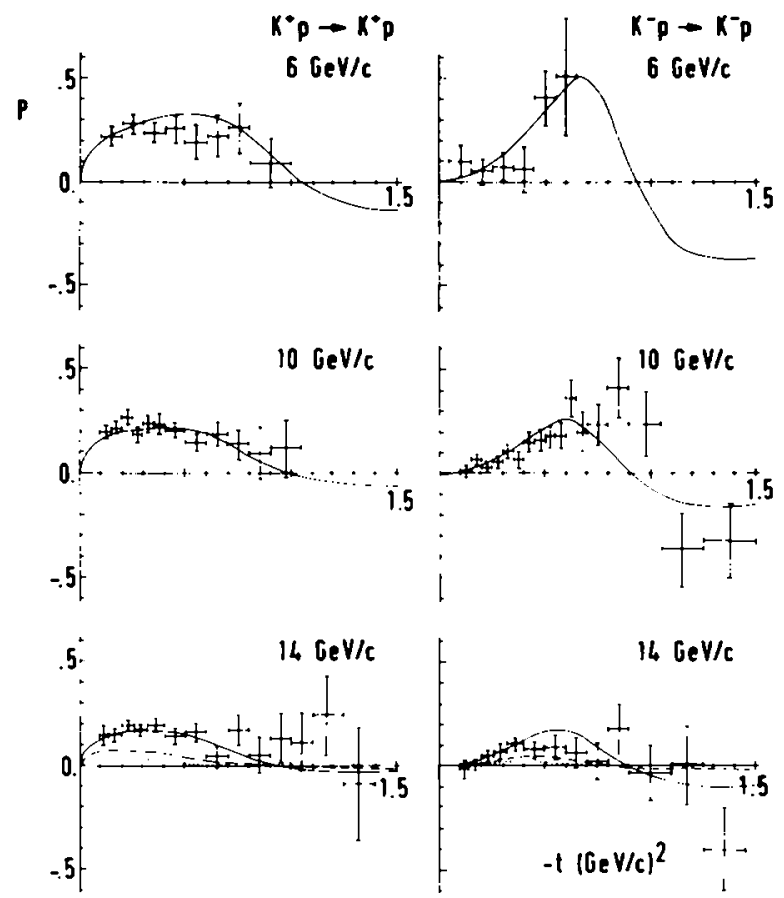

Iig. 16. Polarizations for $K^{\ddagger} p \rightarrow K^{ \pm} p$ calculated from the global fit. Data from ref. [40]. The predicted polarizations at $45 \mathrm{GeV} / c$ and $100 \mathrm{GeV} / c$ are shown by dashed and dotted lines, respectively.

smaller than a fm but still take up a lot of the volume of the hadron) we get

$$
\begin{aligned}
\sqrt{4 B} & \approx 3 \mathrm{GeV}^{-1}, \\
B & \approx \frac{9}{4} \mathrm{GeV}^{-2} .
\end{aligned}
$$

Similarly, if the edge has a full width at half maximum of $d$ it corresponds approximately to

$$
B_{\mathrm{o}} \approx \frac{1}{4} d^{2}
$$

so if $d \approx 2.5 \mathrm{GeV}^{-1}=0.5 \mathrm{fm}$ we have

$$
B_{\mathrm{o}} \approx 1.5 \mathrm{GeV}^{-2}
$$

Also, of course, at some typical energy above a few $\mathrm{GeV} / c$ we expect

$$
R \approx 1 \mathrm{fm}=5 \mathrm{GeV}^{-1}
$$



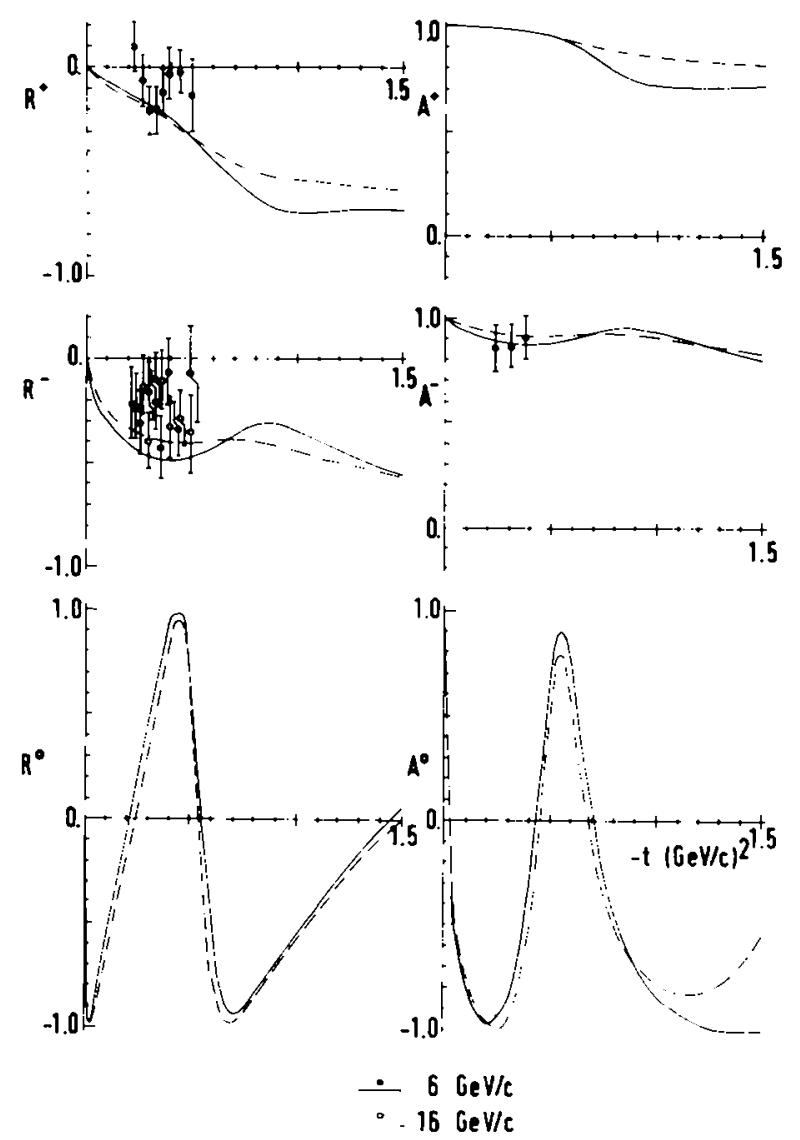

Fig. 17. The $R$ - and $A$-parameters for $\pi^{ \pm} \mathrm{p} \rightarrow \pi^{ \pm} \mathrm{p}$ and $\pi " \mathrm{p} \rightarrow \pi^{\circ} \mathrm{n}$. Data from ref. [41]. The isoscalar-flip amplitude has not been included.

At $p_{\mathrm{L}}=6 \mathrm{GeV} / c$, for example, this would give $\left(R^{2}=R_{\mathrm{o}}^{2} \ln s\right) R_{\mathrm{o}}=3.1 \mathrm{GeV}^{-1}$, while at $60 \mathrm{GeV} / \mathrm{c}$, beyond which most cross sections will not change much, it gives $R_{\mathrm{o}}=2.4 \mathrm{GeV}^{-1}$.

If any of these numbers should deviate significantly from these estimates (say more than about $\pm 1 \mathrm{GeV}^{-2}$ for $B, B_{\mathrm{o}}$ or $\pm 1 \mathrm{GeV}^{-1}$ for $R$ ) we have not got the physics correct. In the present analysis, of course, we meet these requirements. In future applications it is important to obey these constraints.

Presumably (to the extent we understand these things) the same arguments apply for the diffractive production. Thus in the vacuum exchange contributions only the relative size of the edge and center contributions of the pomeron and the relative size of the inelastic intermediate state contribution can be treated as free parameters. There are just two numbers for all $0^{-\frac{1}{2}^{+}}$reactions.

We emphasize here and again later that once we have analyzed some data and 


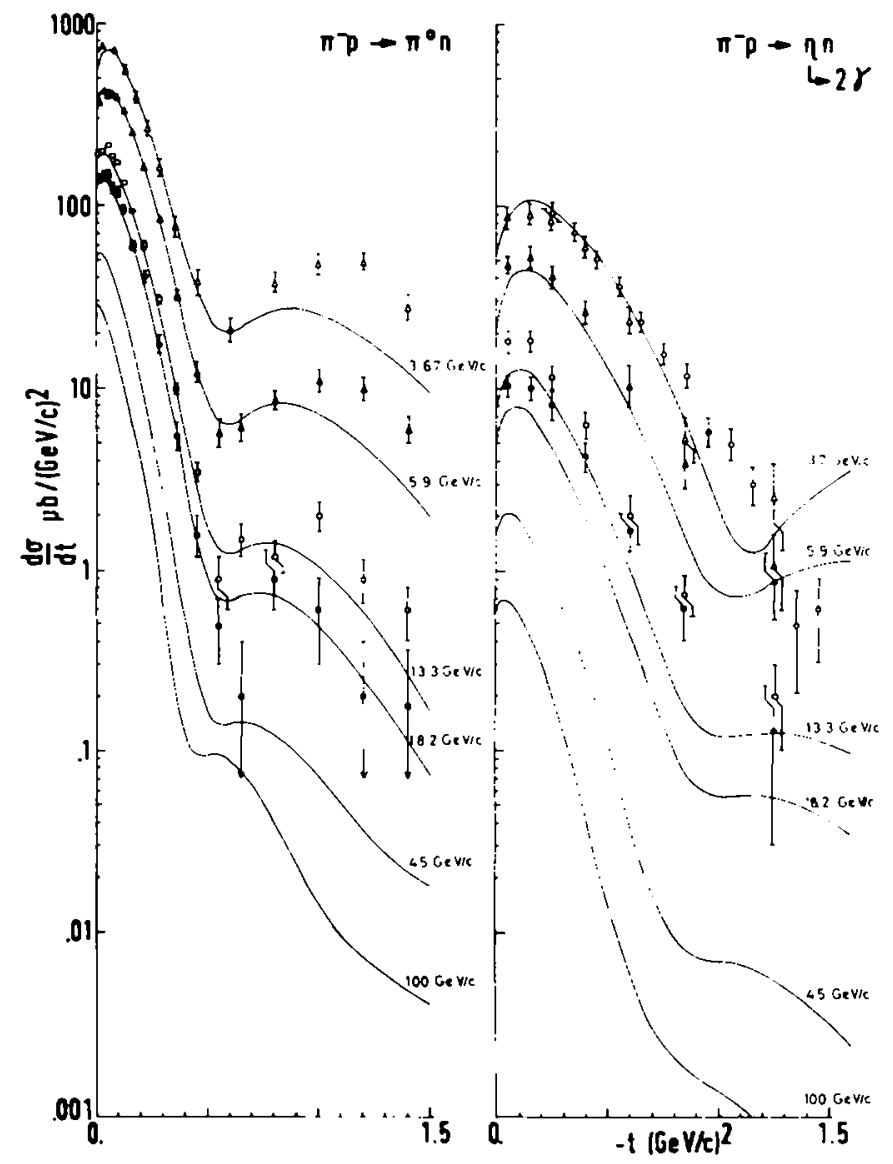

Fig. 18. Global fit differential cross sections for $\pi^{--} p \rightarrow \pi^{\circ} n$ and $\pi^{-} p \rightarrow \eta n$. The theoretical curve for $\eta$-production has been multiplied by the branching ratio $(\eta \rightarrow 2 \gamma) /(\eta \rightarrow$ all modes $)$ which we took to be 0.38 . Data from refs. $[42,43]$.

found values for most of the parameters, one can go to new reactions with very few unknowns.

SU(3) is used, so we assume $\rho$ and $\omega$ have the same trajectories, and $A_{2}$ and $f$ have the same trajectories. Thus the two intercepts $\alpha_{\rho}(0)$ and $\alpha_{A_{2}}(0)$ are parameters. For the $\mathrm{K}^{*}$ and $\mathrm{K}^{* *}$ contributions we use one symmetry breaking parameter, measuring $\alpha_{\rho}(0)-\alpha_{K}(0)=\alpha_{A_{2}}(0)-\alpha_{K^{* *}}(0)$. There is a $d / f$ ratio for flip and for nonflip amplitudes, assumed the same for vector and tensor multiplets, and fixed $a$ priori. The ratio of flip to non-flip coupling is taken to be the same for $\rho$ and $A_{2}$.

Given the pomeron and diffractive production contributions for $\pi \mathrm{N}$ we determine them for $\mathrm{KN}$ by simply scaling their overall magnitude by the ratio $\sigma_{\mathrm{T}}(\mathrm{KN})$ / $\sigma_{\mathrm{T}}(\pi \mathrm{N})$ and for the hypercharge-exchange reactions by scaling $M_{\text {eff }}$ by the average 


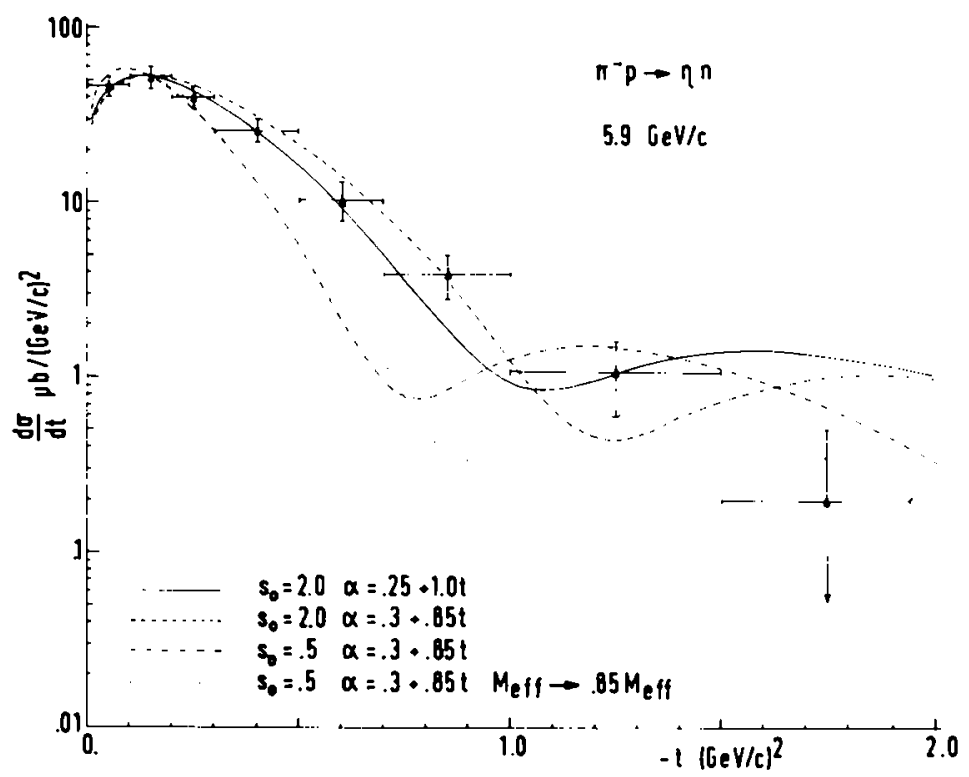

Iig. 19. This illustrates the sensitivity of the $\pi^{-} p \rightarrow \eta n$ cross section to minor changes in the model. It is so sensitive mainly because the tensor exchange has a short range and the final amplitudes depend strongly on the details of the absorption of the low partial waves. The large-t dip in the cross section is mainly dependent on where $\operatorname{Re} M_{+_{-}}=0$, and that can occur any where from $-t \approx 1$ to $-t \approx 1.4$ depending on detailed considerations. Note from this and fig. 18 that we al ways have a large- $t$ dip in $d \sigma / d t$ even though we have no signature zero in the $A_{2}$ contribution.

$\sigma_{\mathrm{T}}(\mathrm{KN})+\sigma_{\mathrm{T}}(\pi \mathrm{N})$. All hypercharge-exchange reactions are always absorbed with the same $M_{\mathrm{cff}}$.

As the interaction gets stronger, however, we believe that one should not just continue to scale up $M_{\text {eff }}$ but instead the shape of the disc in impact parameter changes, with the edge more filled in, closer to a black disc. Thus for the NN reactions we should not just scale but we must fill in the disc, exactly as one would naively hope.

Due to the limitations of both human and computing systems we do not yet have a single comprehensive analysis of all reactions. Instead, we have a unified analysis of $0^{-\frac{1}{2}^{+}} \rightarrow 0^{-\frac{1}{2}^{+}}$reactions, and a separate but essentially compatible analysis of $\mathrm{np} \rightarrow \mathrm{pn}$ and $\mathrm{pp} \rightarrow \overline{\mathrm{n}} \mathrm{n}$, including polarizations. The latter analysis was performed mainly by Vaughn [18] and is a part of a comprehensive analysis of all NN reactions compatible with our present analysis; it will be published separately. For completeness Vaughn has allowed us to include here these processes to show both that the polarizations and the $\pi$-exchange problem can be handled satisfactorily. Thus essentially all common data on two-body reactions can be described in terms of the amplitudes we examine in this paper (net helicity flip $n=0,1,2$ and the evasive amplitude for $n=2$ ).

Another general point which needs emphasis before we examine our results in de- 


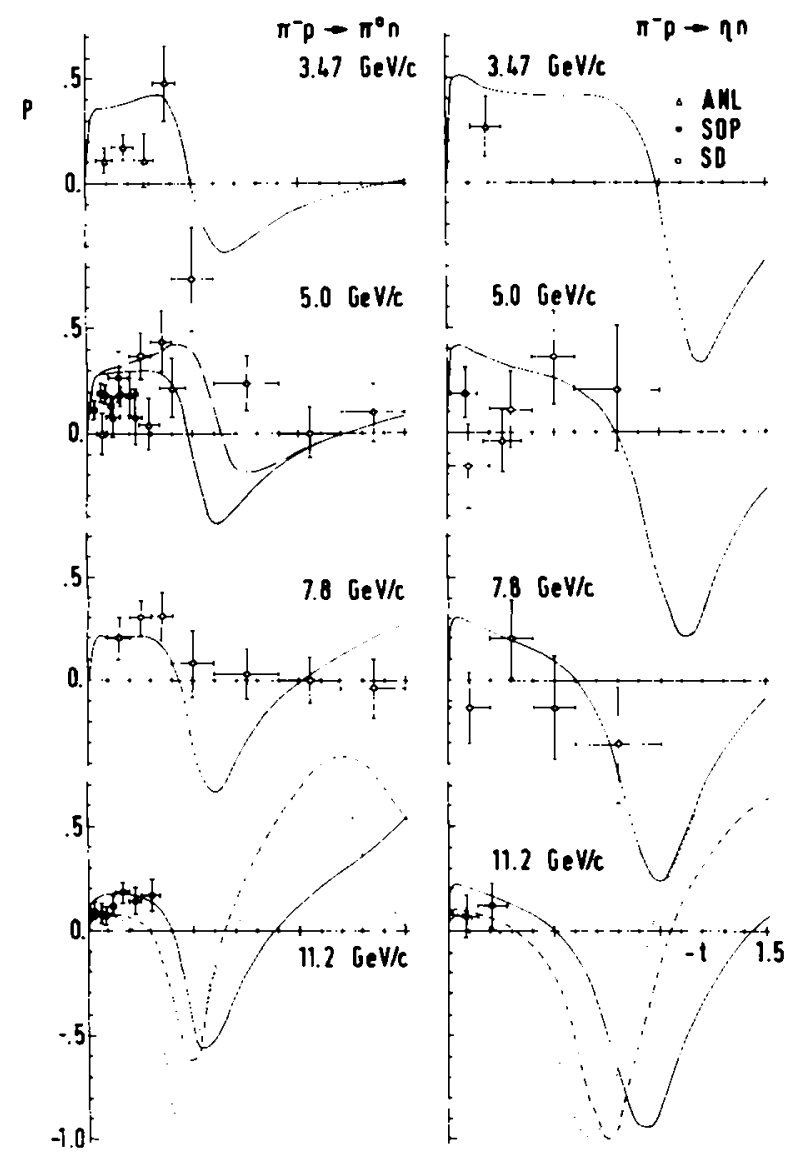

Fig. 20. Global fit polarizations for $\pi^{-} p \rightarrow \pi^{o} n$ and $\pi^{-} p \rightarrow \eta n$. Data from ref. [44]. The dashdotted line at $5 \mathrm{GeV} / \mathrm{c}$ shows a polarization curve if we use the global fit with only the change $\alpha_{\rho}(t)=0.5+0.85 t$, illustrating how sensitive the polarization is to minor changes; with this change the cross sections and elastic polarizations are still well described. Near the dip region the polarization is extremely sensitive to very small changes in the real parts of the amplitudes (where small means by amounts small compared to the magnitude of the amplitude in that region) so that a knowledge of the data in that region will help to fix the details within a model which already has real parts like those in fig. 5 for $\rho$-exchange. Of course, as is well known now, the absence of a large negative spike in the polarization before the $\mathrm{dip}$ in $\mathrm{do} / \mathrm{d} t$ provided the clue that a rotation such as that provided by our complex pomeron was needed to understand the data (see discussion in text). The dashed and dotted lines are predictions for $45 \mathrm{GeV} / \mathrm{c}$ and $100 \mathrm{GeV} / c$ respectively.

tail is the concept of a global fit to the data. We are able to obtain here, with no surprises and with about one parameter per reaction, a rather good description of almost all $0^{-\frac{1}{2}}+$ data for $-t \leqslant 1.5 \mathrm{GeV}^{2}$ and $p_{\mathrm{L}} \geqslant 4 \mathrm{GeV} / c$. As we will discuss below, our model is so simple in its formulation that many aspects of the physics are over- 


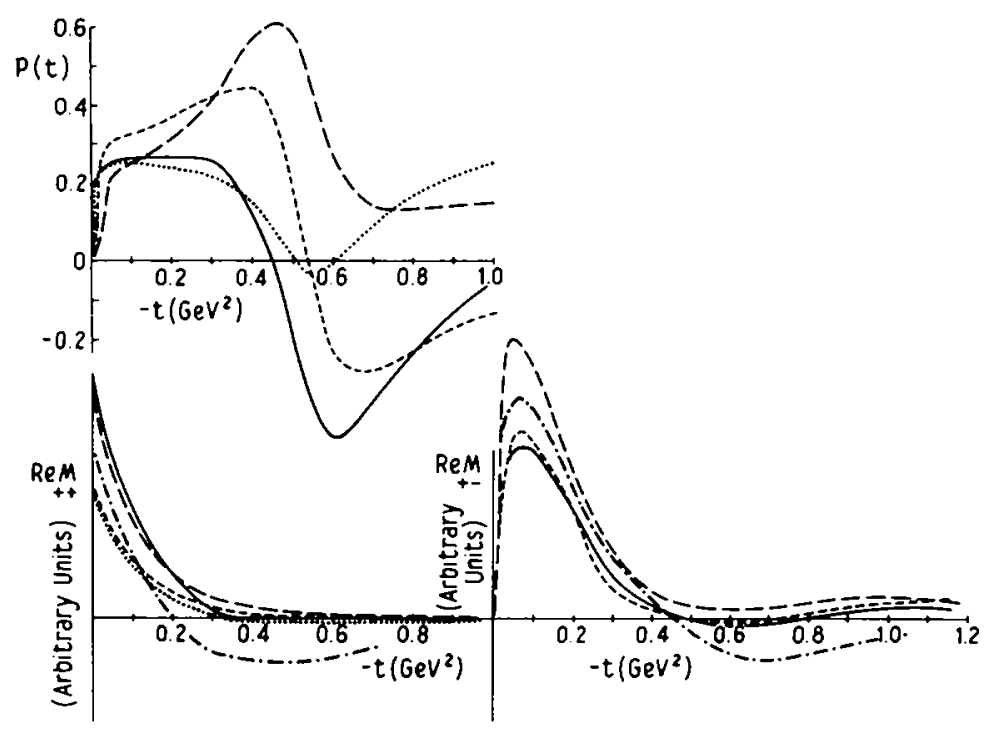

Iig. 21. Four polarizations are shown which are essentially physically equivalent. They differ because of the small differences in the real parts of the amplitudes, shown with matching lines. The imaginary parts of the amplitudes are essentially identical for all of them. Also shown for comparison on the Re $M$ graphs is a typical old strong absorption result for the real parts; it is basically different compared to any of the others and resulted in the deep negative spike in $P(t)$ which has been ruled out by the data.

simplified or left out. A few aspects of the data are not described very well. If we go from the global fit to a local one where we allow small changes in the global parameters we can obtain good fits everywhere. Since we have so few parameters, and so much of the structure is due to the general behavior of our amplitudes, we interpret this as implying that we are not completely missing any essential aspect of the physics.

The $0^{-} \frac{1}{2}^{+} \rightarrow 0^{-\frac{1}{2}^{+}}$reactions. Now we discuss the application of the model to the 16 connected spin $0-\operatorname{spin} \frac{1}{2}$ forward reactions.

As is fairly widely known, fits to experimental data are never unique. In addition to showing the fits we wish to make the following points about the physics involved:

(a) It is possible to obtain a reasonable, simultaneous description of all these reactions, with only the high-lying reggeons $\left(\rho, \mathrm{A}_{2}, \omega, \mathrm{f}\right)$, our pomeron, $\mathrm{SU}(3)$, and our absorptive corrections. No qualitative effects are left out. The forward peak in $t$ and all energies above the resonance region are included. We call this the global fit. Any particular part of the data plays a small role since many cross sections and polarizations are fitted.

(b) If one wants, one can take a smaller segment of the sixteen reactions (e.g., $\pi \mathrm{N}$ ) and, beginning from the global fit, obtain a still better local fit by small changes in parameters. 


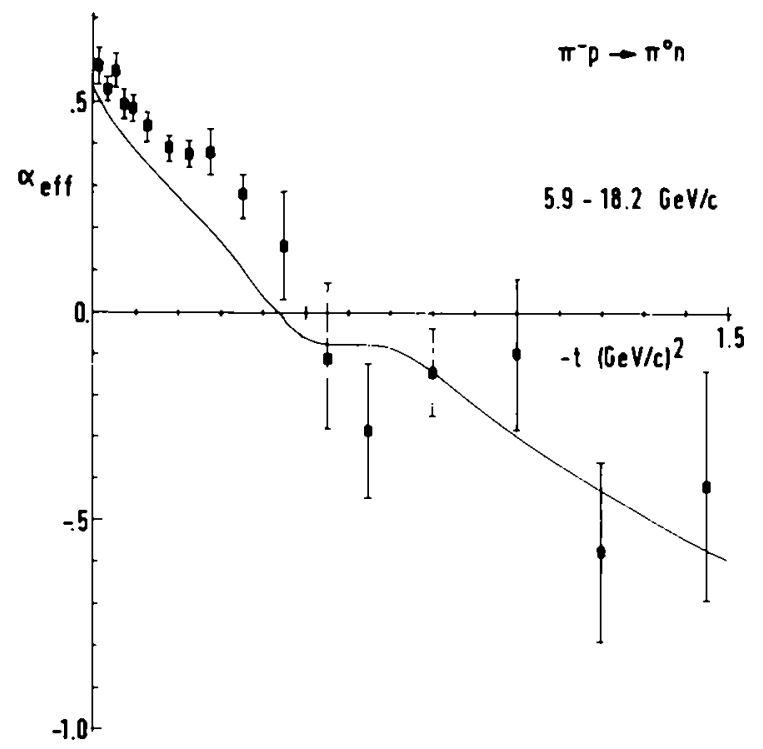

Fig. 22. The global fit $\alpha_{\text {eff }}$ for $\pi^{-} \mathrm{p} \rightarrow \pi^{\circ}$ n over the energy range 5.9 to $18.2 \mathrm{GeV} / c$. The data are from ref. [4]. Note that previous difficulties with shrinkage in this energy range are no longer present (sce discussion in text).

(c) Various results which had been thought to be puzzles, or at least not well un. derstood, can be simply related and interpreted. These include energy dependence of $\sigma_{\mathrm{T}}, \Delta \sigma_{\mathrm{T}}(\pi \mathrm{N}), \Delta \sigma_{\mathrm{T}}(\mathrm{KN})$, relations among crossovers for $\pi \mathrm{N}$ and $\mathrm{KN}$ and their energy dependence, energy dependence of $\pi^{-} \mathrm{p} \rightarrow \pi^{\circ} \mathrm{n}$, the increased slope and shrinkage at small $t$ in $\mathrm{d} \sigma / \mathrm{d} t$ for pp scattering (and $\pi \mathrm{N}, \mathrm{KN}$ as well), line reversed reactions, etc.

(d) In addition to general properties of the model - different range for $\mathrm{V}, \mathrm{T}$ exchange, the effect of the pomeron phase and edge on absorption, the contribution of inelastic states to $M_{\text {eff }}$ - one more (parameter-dependent) property is essential. Just as we do not assume an exchange degenerate residue structure for $\mathrm{V}, \mathrm{T}$ we also do not assume exchange degenerate trajectories. On the contrary, in fact, by specifically not having degenerate trajectories we obtain amplitudes surprisingly degenerate in phase. With $\alpha_{\mathrm{V}}(0)-\alpha_{\mathrm{T}}(0) \approx 0.15 \pm 0.05$ we can understand various qualitative features such as the rise in $\sigma_{\mathrm{T}}\left(\mathrm{K}^{+} \mathrm{p}\right)$ and the behavior of line reversed reactions. In all the following analyses of data we assume as a basic property that $\alpha_{V}(0)-\alpha_{T}(0) \approx 0.15$. This property is essential in understanding a number of aspects of the data, including line reversed reactions, the rise in $\Delta \sigma_{\mathrm{T}}\left(\mathrm{K}^{+} \mathrm{p}\right)$, and several polarizations.

We have illustrated the results of the model compared with representative data over as wide a range of energies as data exists, but we have not tried to include every piece of data. This is simply to make the diagrams less cluttered; our fit to data we have omitted are generally similar. 

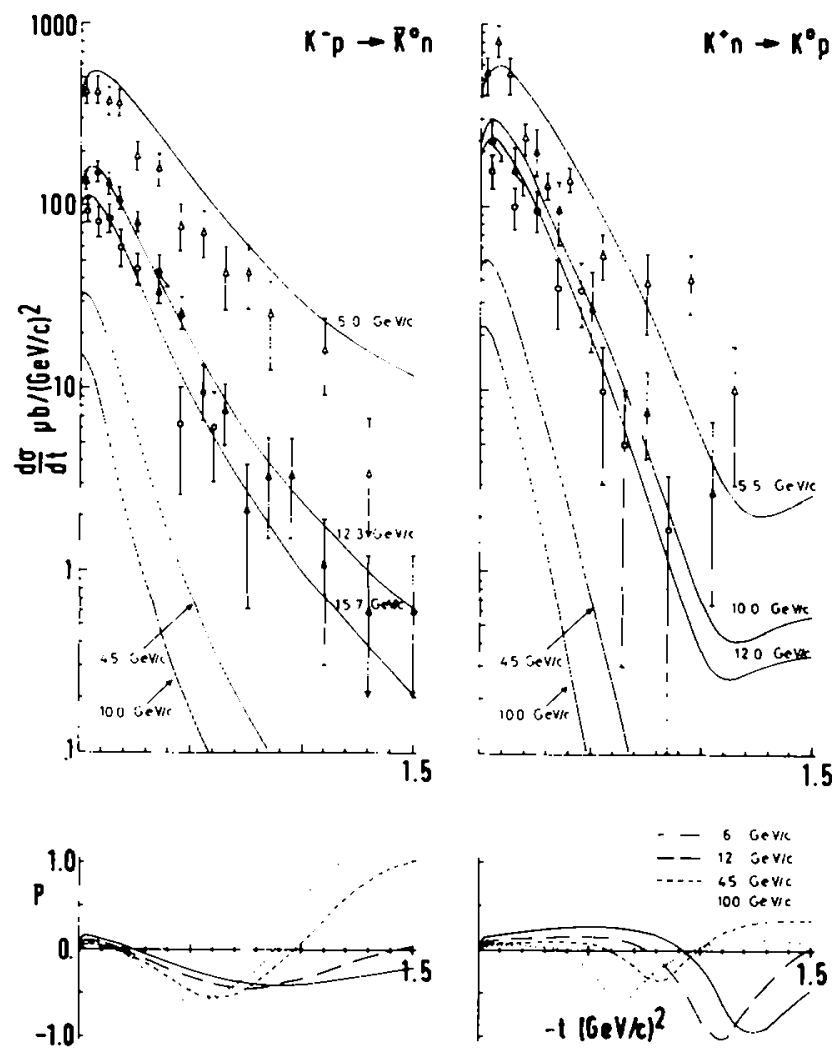

Fig. 23. Global fit diuerential cross sections for the line-reversed reactions $\mathrm{K}^{-} \mathrm{p} \rightarrow \overline{\mathrm{K}}^{\mathrm{O}} \mathrm{n}$ and $K_{n} \rightarrow K^{0}$. Data from refs. $[45,46]$. The predicted polarizations are also shown. Note that at higher energies we predict the real reaction $K^{+} n \rightarrow K^{0} p$ to have a larger cross section than the rotating one; a similar prediction holds for any line reversed pair. See fig. 24 and section in text.

Our procedure is essentially as follows. We posses most information about the $\pi \mathrm{N}$ system, particularly at $P_{\text {lab }}=6 \mathrm{GeV} / c$. This determines most of our parameters. The magnitude of the pomeron plus an f-exchange (absorbed with $S_{\text {eff }}$ for $\pi \mathrm{N}$ ) is fixed by the magnitude of the non-flip, isoscalar amplitude of Halzen and Michael [19], a knowledge of the s-dependence of $\sigma_{\text {tot }}(\pi \mathrm{p})$ also constraining $\operatorname{Im} P(t=0)$ and $\operatorname{Im} f(t=0)$. The phase of the pomeron is chosen such that the absorbed $\rho$ gives the Halzen and Michael isovector amplitude. These also fix $\beta_{++}$for $\rho$-exchange. The $\alpha_{0}$ for the $\rho$ largely determines the $s$-dependence of $\mathrm{d} \sigma / \mathrm{d} t\left(\pi^{-} \mathrm{p} \rightarrow \pi^{\mathrm{o}} \mathrm{n}\right)$ near $t=0$. We choose a universal $s_{\mathrm{o}}=0.5$ (except possibly for the $\mathrm{A}_{2}$ ) and $\alpha^{\prime}=1.0$, these being about the values obtained in our earlier analysis [12].

We fix the $\mathrm{A}_{2}$ pole parameters $\left(\alpha_{0}, s_{0}, \beta_{+ \pm}\right)$to give a reasonable representation of $\pi^{-} \mathrm{p} \rightarrow \eta \mathrm{n}$, using the same absorption as for $\pi \mathrm{N}$. 


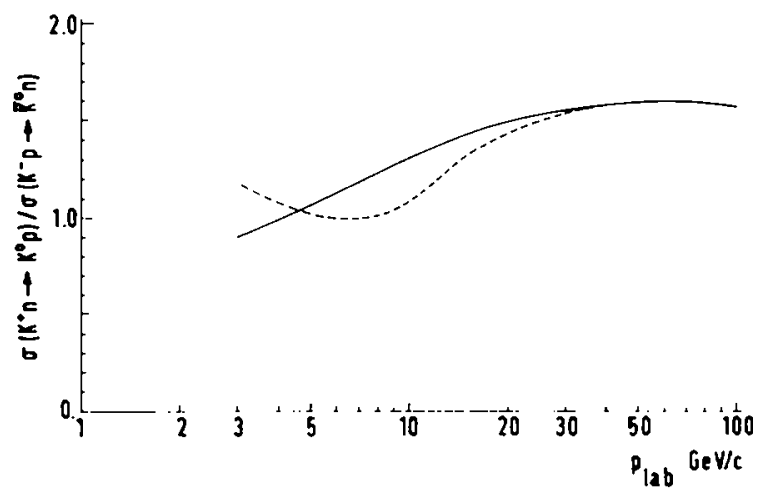

Fig. 24. The solid curve shows the ratio we predict in the model for $R=\sigma\left(\mathrm{K}^{+} \mathrm{n} \rightarrow \mathrm{K}^{\circ} \mathrm{p}\right) / \sigma\left(\mathrm{K}^{-} \mathrm{p} \rightarrow \overline{\mathrm{K}}^{0} \mathrm{n}\right)$, for the integrated cross sections. A similar result holds for the differential cross sections at small $t$. The dashed curve shows a conjectured behavior (for the same ratio) which has the same high-energy physics built in but takes into account that as the energy decreases toward threshold the strength of the absorption will decrease rather than continue to grow as in the model; the dashed curve is meant to be a good guess for the actual behavior. Note that we expect $R \geq 1$ at all energies and significantly larger than 1 at higher energies.
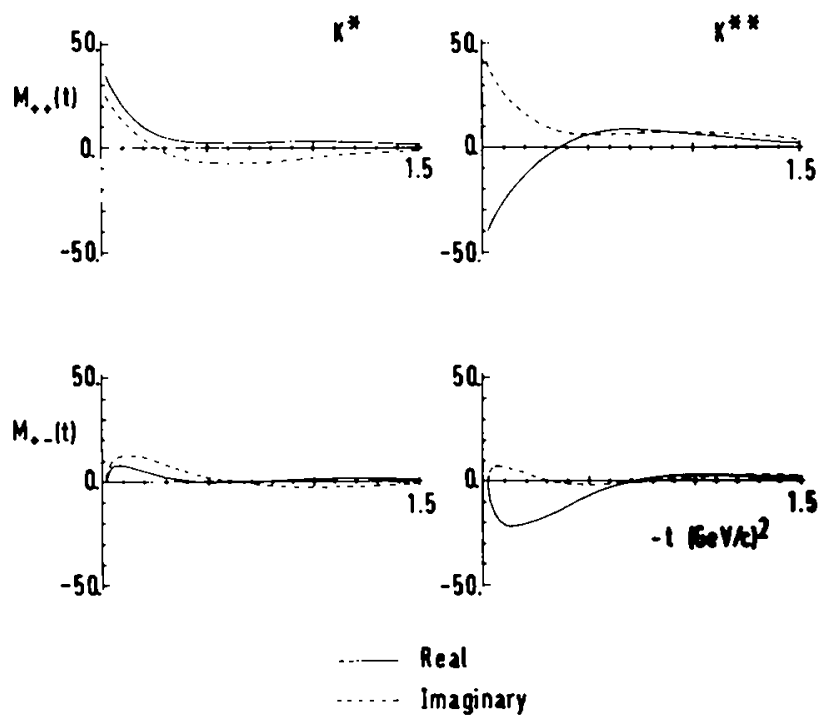

Fig. 25. Global fit non-flip and flip amplitudes for $\mathrm{K}^{*}$ and $\mathrm{K}^{* *}$ exchange in $\pi^{+} \mathrm{p} \rightarrow \mathrm{K}^{+} \mathrm{\Sigma}^{+}$.

Fixing the magnitude of $\omega_{++}$to give $\Delta \sigma_{\text {tot }}(\mathrm{Kp})$, and scaling the pomeron and $\mathrm{D}$ in the ratio of total cross sections with $\sigma_{\mathrm{T}}(\mathrm{KN}) / \sigma_{\mathrm{T}}(\pi \mathrm{N})=0.8$, we then predict $\mathrm{KN}$ reactions with no further parameters using $S U(3)$.

For the hypercharge-exchange reactions we scale $S_{\text {eff }}$ by $\frac{1}{2}\left(\sigma_{\text {tot }}(\pi \mathrm{p})+\sigma_{\text {tot }}(\mathrm{Kp})\right) / \sigma(\pi \mathrm{p})$ 


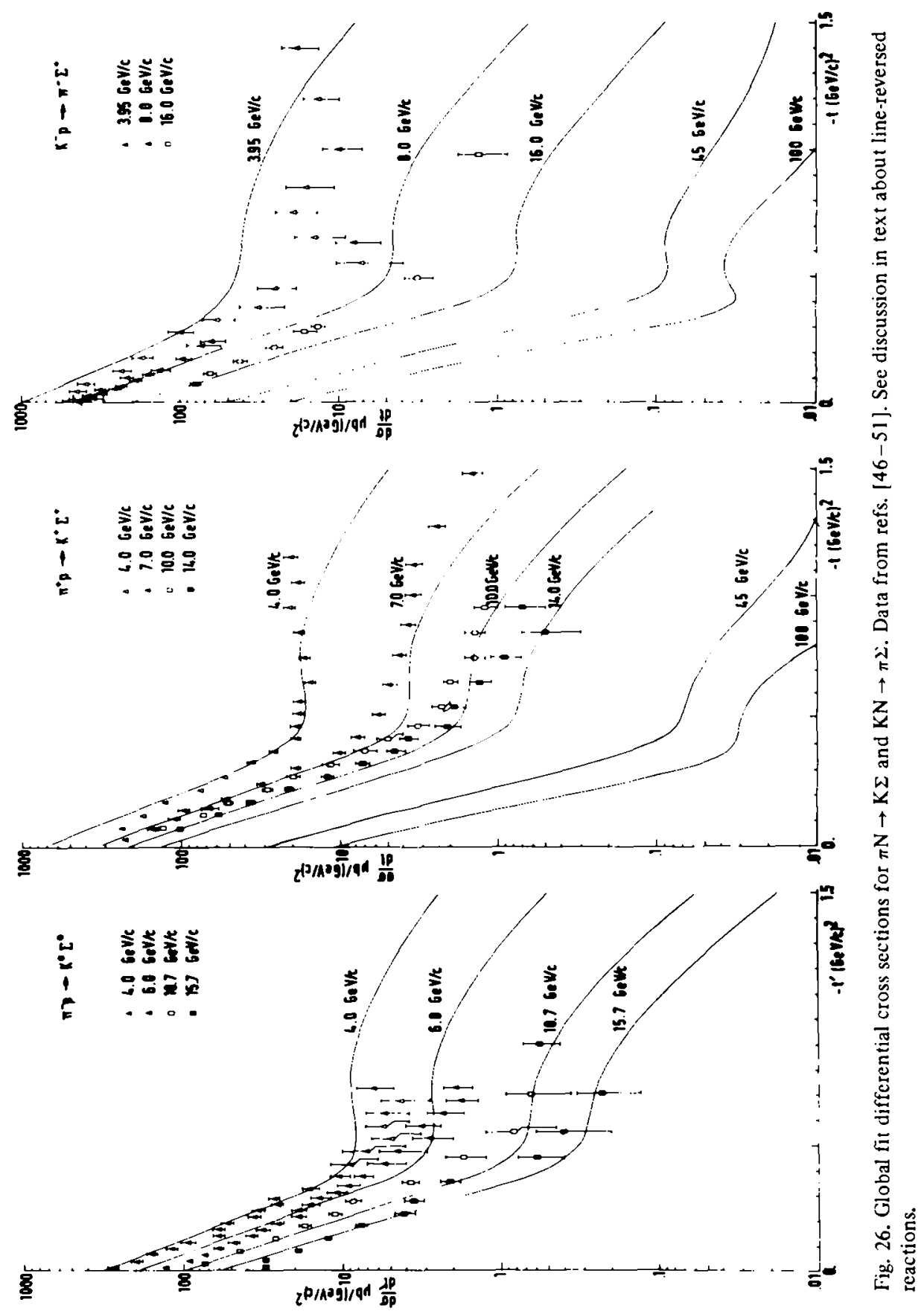




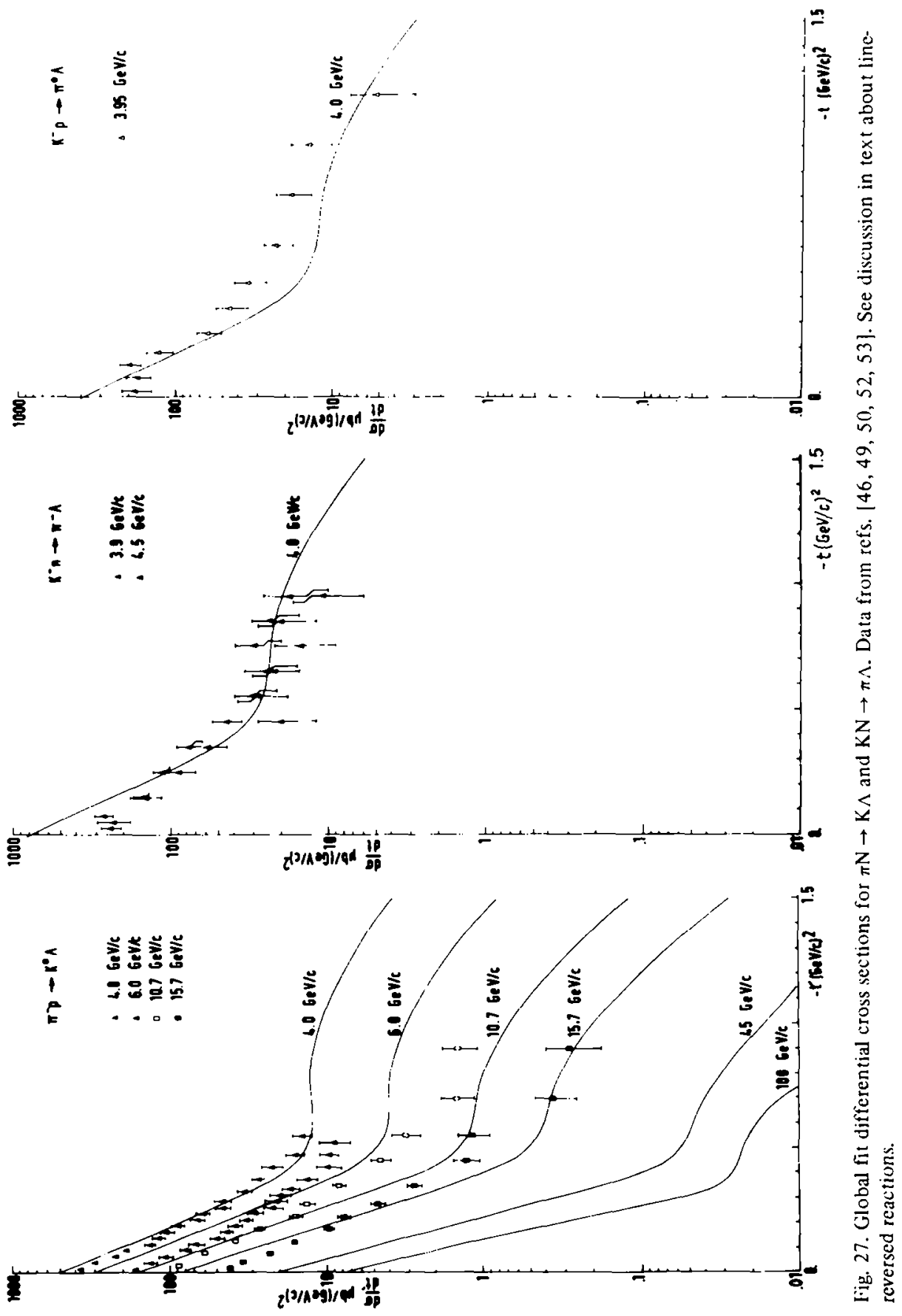


and vary $\alpha_{0}$ for $K^{*}$ and $K^{* *}$ and the $d / f$ ratio. The $d / f$ ratios are essentially as given by Michael [2], but varied slightly since our assumptions are slightly different from theirs.

The results are shown in figs. 5-27. A good deal of discussion is given in the figure captions. The basic effects one needs to understand the results are listed below. Some are general properties of any absorption model and others are only a consequence of our model - we will try to distinguish.

(i) At finite energies and small $t$ an absorbed amplitude has a higher $\alpha^{\mathrm{eff}}(t)$ than the equivalent input $\alpha(t)$. To see this, represent the amplitude as

$$
\begin{aligned}
M & \sim \text { Polc }- \text { Abs } \\
& \sim s^{\alpha}[1 \cdot c / \ln s],
\end{aligned}
$$

where at small $t$ the absorption correction falls with energy faster than the pole and we have typified that extra fall-off by const/ln s. (This form is only really correct for non-flip amplitudes at $t=0$, but it is not misleading at small $t$ for all amplitudes for purposes of our argument.) Then as $s$ increases the factor in brackets increases, so very crudely

$$
[1-c / \ln s] \sim s^{\epsilon},
$$

where $\epsilon$, while depending slowly on energy, is positive (and almost constant over a few $\mathrm{GeV} / \mathrm{c}$ ). Thus

$$
\alpha^{\mathrm{eff}} \equiv \alpha+\epsilon>\alpha
$$

Further, since the absorption is stronger for non-flip than flip amplitudes, one has $\alpha_{++}^{\text {eff }}>\alpha_{+-}^{\text {eff }}>\alpha$. This effect accounts for slower fall-off of $\Delta \sigma_{\mathrm{T}}(\pi \mathrm{N})$ than $\mathrm{d} \sigma / \mathrm{d} t\left(\pi^{-} \mathrm{p} \rightarrow \pi^{\mathrm{O}} \mathrm{n}\right)$ since $\Delta \sigma_{\mathrm{T}}$ only samples $\alpha_{\rho}^{\text {eff }}(t=0)$ in the non-flip amplitude while the $\pi^{--} \mathrm{p} \rightarrow \pi^{\mathrm{o}} \mathrm{n}$ cross section al ways samples away from $t=0$ and the flip amplitude. Our global fit (fig. 21) has $\alpha_{\rho}^{\text {eff }}(t=0)-\alpha_{\rho}(t=0)=0.12$, i.c., $\alpha_{\rho}^{\text {eff }}(0)=0.57$, and by paying attention to $\Delta \sigma_{\mathrm{T}}$ in the fitting one can casily get $\alpha_{\rho}^{\text {eff }}(0)=0.63$ (the Serpukhov result is $0.67 \pm 0.05$ ).

(ii) At the same time, however, since $\sigma_{T}(K N)<\sigma_{\Gamma}(\pi N)$ the absorption in KN reactions is simply less than in $\pi \mathrm{N}$ (fig. 7), so $\alpha_{\omega}^{\text {cff }}(0)$ is not so high and $\Delta \sigma_{\mathrm{T}}(\mathrm{KN})$ must fall faster than $\Delta \sigma_{\mathrm{T}}(\pi \mathrm{N})$ in the absorption model.

(The only objection a reader could raise here is why $\Delta \sigma_{\mathrm{T}}\left(\mathrm{p}^{+} \mathrm{p}\right)$ does not fall still slower since $\sigma_{T}(N N)>\sigma_{T}(\pi N)$. Since we have not yet had time to do a full simultaneous treatment of all NN reactions and all meson-baryon ones we are not absolutely sure that this is not an objection, but we think it is not because the main effect of increasing $\sigma_{\mathrm{T}}$ as one gets nearer to the unitarity limit is to fill out the disc rather than blacken it more; this makes the real part even more important in the absorption, and brings both $\Delta \sigma_{\mathrm{T}}$ and the crossover zero closer to $\mathrm{KN}$ than to $\pi \mathrm{N}$. We will explain this in more detail below and in ref. [18].) 
This result is also connected to the crossover zero, which is mainly due to a zero in $\operatorname{Im} \rho$ or $\operatorname{Im} \omega$ near $-t=0.2 \mathrm{GeV}^{2}$. This zero arises from absorption, and since $\sigma_{\mathrm{T}}(\pi \mathrm{N})>\sigma_{\mathrm{T}}(\mathrm{KN})$ the absorption is stronger in $\pi \mathrm{N}$ and the crossover zero is expected to be closer to $t=0$ in $\pi \mathrm{N}$ than it is in $\mathrm{KN}$ (for NN sec remark just above and discussion below). Since the strength of the absorption decreases with energy (see discussion under gencral structure) the crossover zero will move out in $t$ with increasing energy up to some energy (at least to $p_{\mathrm{L}} \sim 30 \mathrm{GeV} / c$ ) and then the Regge shrinkage will take over and the zero will slowly move back in. How far it moves is model dependent; we would expect a shift outward of about $\Delta t=0.1 \mathrm{GeV}^{2}$ from $6 \mathrm{GeV}_{i} c$ to $20 \mathrm{GeV} / c$.

(iii) By pursuing these same arguments one can see that in our model the rise $\sigma_{\mathrm{T}}\left(\mathrm{K}^{+} \mathrm{p}\right)$ occurs naturally and is due solely to reggeons. At the same time one can sec the origin of the forward real parts of the clastic amplitudes.

Fig. 9a shows the contribution of the f-exchange in $\mathrm{K}^{+} \mathrm{p}$ elastic scattering at $t=0$ in an Argand plot (all the diagrams in fig. 9 are meant to illustrate qualitative effects and may not represent precise numerical values). Now, around $10 \mathrm{GeV} / c$ (say) we know that $\sigma_{\mathrm{T}}\left(\mathrm{K}^{+} \mathrm{p}\right)$ is constant in energy, so there it must happen that $\operatorname{Im}(\omega)+$ $\operatorname{Im}(f)=0$. The input even-signature $f$-pole with $\alpha(0) \sim \frac{1}{3}$ has a phase like $-e^{-\cdots \frac{1}{4} i \pi}=$ $\mathrm{e}_{6}^{\frac{5}{6} i \pi}$ and our absorption rotates it away from the real axis, giving $|\operatorname{Re}(\mathrm{f})|<|\operatorname{Im}(\mathrm{f})|$. Thus we can draw fig. $9 \mathrm{~b}$ showing $f$ - and $\omega$-contributions, with $\operatorname{Im}(\omega)+\operatorname{Im}(f)=0$.

Then we will get $\operatorname{Re} M\left(\mathrm{~K}^{+} \mathrm{p}\right)$ large and negative since $\operatorname{Re}(\mathrm{f})$ and $\operatorname{Re}(\omega)$ add. This is in agreement with experimental data. This shows that we chose the correct sign for the $\mathrm{f}$ - and $\omega$-contributions; otherwise they would give a positive $\operatorname{Re} M\left(\mathrm{~K}^{+} \mathrm{p}\right)$.

Now, however, as the energy increases the f-contribution will go away faster than the $\omega$-contribution because it is a general property of our model that $\alpha_{\mathrm{T}}^{\text {eff }}<\alpha_{\mathrm{V}}^{\mathrm{eff}}$. Thus at some energy, higher than the energy where $\sigma_{\mathrm{T}}\left(\mathrm{K}^{+} \mathrm{p}\right)$ behaves as a constant, $\sigma_{\mathrm{T}}\left(\mathrm{K}^{+} \mathrm{p}\right)$ must rise to a higher constant asymptotic value, just from the way the reggeons go away with energy ( $\mathrm{Im}$ reggeons/Im pomeron $<0$ ). Without any effort one obtains rises of $\frac{1}{4} \mathrm{mb}$, and the Serpukhov data [20] can be fit (a rise of $\frac{1}{2} \mathrm{mb}$ up to $50 \mathrm{GeV} / c$ ) if one desires.

Exactly the same argument and statement apply in pp scattering. There, however, for reasons connected with coupling strengths and having no simple connection to $s$ - and $t$-dependence or absorption models, at energies in the $10-20 \mathrm{GeV} / \mathrm{c}$ range one still has $\sigma_{\mathrm{T}}$ falling with $s$. The f-contribution, while falling faster, is still larger than $\omega$. Thus the rise to a constant limit is displaced to higher energies for pp, and will be correspondingly small because the size of the reggeons is smaller at higher $s$. Its precise size will be estimated in the NN analysis. (In this analysis we have assumed that the pomeron contributes a constant $\sigma_{\mathrm{T}}$. If that should turn out to rise with energy, its rise will be in addition to that of the reggeons.)

The situation for the other elastic reactions can be seen from fig. 9 . For $K^{\prime} p \rightarrow K^{-} p$ the sign of the $\omega$-cxchange reverses since it is an odd-charge conjugation exchange.

Thus necessarily we have $\operatorname{Re}\left(\mathrm{K}^{--} \mathrm{p}\right) / \operatorname{Im}(\mathrm{K} p)>0$ and a falling $\sigma_{\mathrm{T}}$. For $\pi^{+} \mathrm{p}$ it is as in fig. 9. For $\pi^{-} \mathrm{p} \sigma_{\mathrm{T}}$ can only fall, while for $\pi^{+} \mathrm{p}$ a small rise could eventually oc- 
cur; it is a detail of the numbers whether one can see the reggeons at the energy where $\operatorname{Im}(\rho)>\operatorname{Im}(\mathrm{f})$. For the baryons $\bar{p} p$ will always fall, while pp could rise somewhat.

(iv) Returning to the argument of (i) just above, we emphasize that amplitudes with different net helicity flip $n$ will fecl different strength of absorption in any absorption model, and thus different helicity amplitudes will have different $\alpha$ eff. In particular, for $\pi \mathrm{p} \rightarrow \pi^{\mathrm{l}} \mathrm{n}, \alpha_{++}^{\mathrm{eff}}(t)$ will be considerably above $\alpha_{\rho}(t)$, while $\alpha_{+-}^{\text {eff }}(t)$ will only be a little above $\alpha_{\rho}(t)$ since the flip amplitude feels less absorption. Consequently, $\mathrm{d} \sigma\left(\pi^{-} \mathrm{p} \rightarrow \pi^{\mathrm{o}} \mathrm{n}\right) / \mathrm{d} t$ or $\sigma\left(\pi^{-} \mathrm{p} \rightarrow \pi^{\mathrm{o}} \mathrm{n}\right)$ will fall faster with energy than $\Delta \sigma_{\mathrm{T}}\left(\pi^{ \pm} \mathrm{p}\right)$ since the latter only sees $\alpha_{++}^{\text {cff }}(0)$, the maximum value. This is observed at Serpukhov [21]. As $s$ increases $\alpha^{\text {eff }} \rightarrow \alpha$ and the two will come together, $\Delta \sigma_{\mathrm{T}}$ falling faster as $\alpha_{++}^{\mathrm{eff}}(0)$ decreases, and $o\left(\pi^{\cdots} \mathrm{p} \rightarrow \pi^{\mathrm{O}} \mathrm{n}\right)$ falling more slowly as the non-flip amplitude becomes more important relative to flip. For a similar reason the forward turnover in $\mathrm{d} \sigma\left(\pi^{-} \mathrm{p} \rightarrow \pi^{\circ} \mathrm{n}\right) / \mathrm{d} t$ will slowly turn into a peak, with zero slope at $t=0$ at some energy above $45 \mathrm{GeV} / c$ (fig. 18). (Note that we have assumed a ratio of flip to non-flip $\rho$-coupling such that the poles alone give a peak since the absorption always reduces the non-flip pole more than the flip; this is the ratio we need to describe the $\pi \mathrm{N}$ data.)

In particular, as shown in fig. 11 , we find $\Delta \sigma_{T}(\pi \mathrm{N}) \sim s^{-0.40}, \sigma\left(\pi \mathrm{p} \rightarrow \pi^{\mathrm{O}} \mathrm{n}\right) \sim s^{-1.20}$, $\mathrm{d} \sigma\left(\pi^{-} \mathrm{p} \rightarrow \pi^{\circ} \mathrm{n}\right) / \mathrm{d} t \sim s^{-0.96}$ at $t=0$ (the difference between $\mathrm{d} \sigma / \mathrm{d} t$ at $t=0$ and $\Delta \sigma_{\mathrm{T}}$ is due to the faster fall-off of the real part of the $\rho$-amplitude since the real part is absorbed less than the imaginary part). The Serpukhov values [20] are $s \quad 0.35, s-1.09$ and $s^{-0.84}$ respectively, with $1015 \%$ crrors on the exponents.

In addition, as mentioned above, we necessarily find that $\Delta \sigma_{\mathrm{T}}\left(\mathrm{K}^{ \pm} \mathrm{p}\right)$ falls faster with energy than $\Delta \sigma_{\mathrm{I}}\left(\pi^{ \pm} \mathrm{p}\right)$, since the amount of absorption is less for $\mathrm{K} . \mathrm{Y}$ than for $\pi \mathrm{N}$ and thus $\alpha_{\rho, \pi \mathrm{N}}^{\mathrm{eff}}>\alpha_{\omega, \mathrm{KN}}^{\mathrm{eff}}$ even though $\alpha_{\rho}(t)=\alpha_{\omega}(t)$. Numerically we get $\Delta \sigma_{\mathrm{T}}\left(\mathrm{K}^{ \pm} \mathrm{p}\right) \sim s \quad 0.48$ while $\Delta \sigma_{\mathrm{T}} \sim s^{-0.40}$. While these numbers are not as different as the Serpukhov data [20] suggest, $\left(s^{-0.56}\right.$ and $\left.s \cdot 0.33\right)$, they are significantly different in the correct direction and are actually not inconsistent with the experimental data, as can be seen in fig. 10. By fitting we could increase these differences from 0.08 to perhaps 0.14 , but a larger difference would probably require $\alpha_{\omega}(0)<\alpha_{\rho}(0)$. (Since $m_{\omega}^{2}-m_{\rho}^{2} \approx 0.03$ one could perhaps reasonably expect to lower the $\omega$ by 0.03 more.)

(v) Now we consider the structure of polarizations. We can correlate the structure in $\pi^{\cdots} \mathrm{p} \rightarrow \pi^{\mathrm{O}} \mathrm{n}, \pi^{ \pm} \mathrm{p} \rightarrow \pi^{ \pm} \mathrm{p}, \mathrm{K}^{ \pm} \mathrm{p} \rightarrow \mathrm{K}^{ \pm} \mathrm{p}, \mathrm{pp} \rightarrow \mathrm{pp}, \mathrm{np} \rightarrow \mathrm{pn}$ and $\overline{\mathrm{p}} \mathrm{p} \rightarrow \overline{\mathrm{n}} \mathrm{n}$.

In fig. 20 we show $P\left(\pi^{-} p \rightarrow \pi^{\circ} n\right)$. It has been widely pointed out that the predictions of older absorption models were qualitatively wrong here, giving a large negative polarization for $-t \lesssim 0.5 \mathrm{GeV}^{2}$. The rotation of the absorption by Re pom removes that problem here as we have discussed before - essentially the effect is to have $\operatorname{Im} \rho=0$ before $\operatorname{Re} \rho=0$.

Polarizations are very sensitive things in models. For example, the widely different polarizations in fig. 21 come from a set of almost equal amplitudes whose real parts are shown there. The CERN measurements [23] that showed no deep dip in 
$P\left(\pi^{-} \mathrm{p} \rightarrow \pi^{\circ} \mathrm{n}\right)$ were important and taught us a lot, but the current disagreement between the CERN [23] and Argonne [24] measurements is not important. Rather, the Argonne experiment confirms that the polarization is not large and negative before the dip in $\mathrm{d} o / \mathrm{d} t$. The difference between the two experiments can be interpreted as being whether $\operatorname{Re} M_{++}^{1}$ actually has a zero near $-t \sim 0.35$ and stays slightly negative (the Argonne case) or almost vanishes but then stays slightly positive (the CERN case). The basic physics is insensitive to this distinction, so it should not be a matter of great concern.

Obtaining the double zero in the elastic $\pi^{ \pm} \mathrm{p}$ polarizations [25] has long been con. sidered a great problem for models without signature zeros in the Regge pole. The solution, which we have previously discussed (ref. [12]), is that both contributions $\operatorname{Im} P \operatorname{Re} \rho_{+}$, and $\operatorname{Re} P \operatorname{Im} \rho_{+_{-}}$are important, with both containing a double-zerolike structure. For the former it arises because $\operatorname{Im} P$ is monotonic while $\operatorname{Re} \rho_{+_{-}}$has a double zero because of the rotated absorption; for the latter the double zero is due to a single zero in each factor at nearby $t$-values. In both cases the double zero arises from the effects of $\operatorname{Re} P$, once from its affect on the absorption and once explicitly due to its zero.

For $K^{ \pm} \mathrm{p}$ polarizations [25] we automatically get the nice result which has been considered a success of dual models, that $P\left(\mathrm{~K}^{+} \mathrm{p}\right)$ rises rapidly away from $t=0$, while $P\left(\mathrm{~K}^{-} \mathrm{p}\right)$ has an extra power of $t$ and rises slowly. It is clear from the amplitudes in fig. 5 why this occurs our reggeon contribution to $K^{+} p$ is indeed mainly real and our reggeon contribution to $\mathrm{K}^{-} \mathrm{p}$ is indeed oscillating and mainly imaginary at small $t$, while the polarization is due to the interference of these with the mainly imaginary pomeron.

Another interesting point concerns the polarizations [25] in the exotic channels $\mathrm{K}^{+} \mathrm{p} \rightarrow \mathrm{K}^{+} \mathrm{p}$ and $\mathrm{pp} \rightarrow \mathrm{pp}$ near $-t=1 ; \mathrm{K}^{+} \mathrm{p}$ appears to have a single zero there and $\mathrm{pp}$ has a double zero. In the conventional exchange degenerate models with an imaginary pomeron and real reggeon contribution the simple approaches have no zeros in either the pomeron or the reggeon and no zero near $-t=1$ in the polarization; more complicated ones will put a zero structure in the (real) reggeon contribution, which will then produce the same result in both $\mathrm{K}^{+} \mathrm{p}$ and $\mathrm{pp}$. In our analysis for $\mathrm{K}^{+} \mathrm{p}$ one has $\operatorname{Im}$ (reggeons) $\approx 0$ since $\sigma_{\mathrm{T}}$ is flat, so the polarization is indeed the product of $\operatorname{Im} P$ with $\operatorname{Re} M_{+}^{1}$, and as shown in fig. $5, \operatorname{Re} M_{+}^{1}$ indeed has a normal absorptive zero at $-t \approx 1$ (farther out than for the $\rho$ alone because of the shorter-range tensor mesons). Thus $P\left(\mathrm{~K}^{+} \mathrm{p}\right)$ is expected to have a single zero near $-t=1$. For $\mathrm{pp}$ on the other hand, $\sigma_{\mathrm{T}}$ is decreasing so at $t=0 \mathrm{Im}$ (reggeons) is positive relative to $\mathrm{Im}$ (pomeron) and significant. As $-t$ increases, Im (reggeons) has a zero, and one can work out that for $-t \sim 0.8$ one has a cancellation between $\operatorname{Im}$ (reggeons) and $\operatorname{Im} P$ giving a zero in $\operatorname{Im} M_{++}^{0}$. The zero in $\operatorname{Re} M_{+-}^{1}$ is still there, and so $P(\mathrm{pp})$ is expected to show an approximate double zero.

Our model also allows us to understand for the first time the polarization [26] in $\mathrm{np} \rightarrow \mathrm{pn}$ and predict reasonably well the polarization in $\overline{\mathrm{p}} \mathrm{p} \rightarrow \overline{\mathrm{n}} \mathrm{n}$ as shown in fig. 30 . Again here we are quoting preliminary results from Vaughn's NN calculations; the complete details will soon be published. 

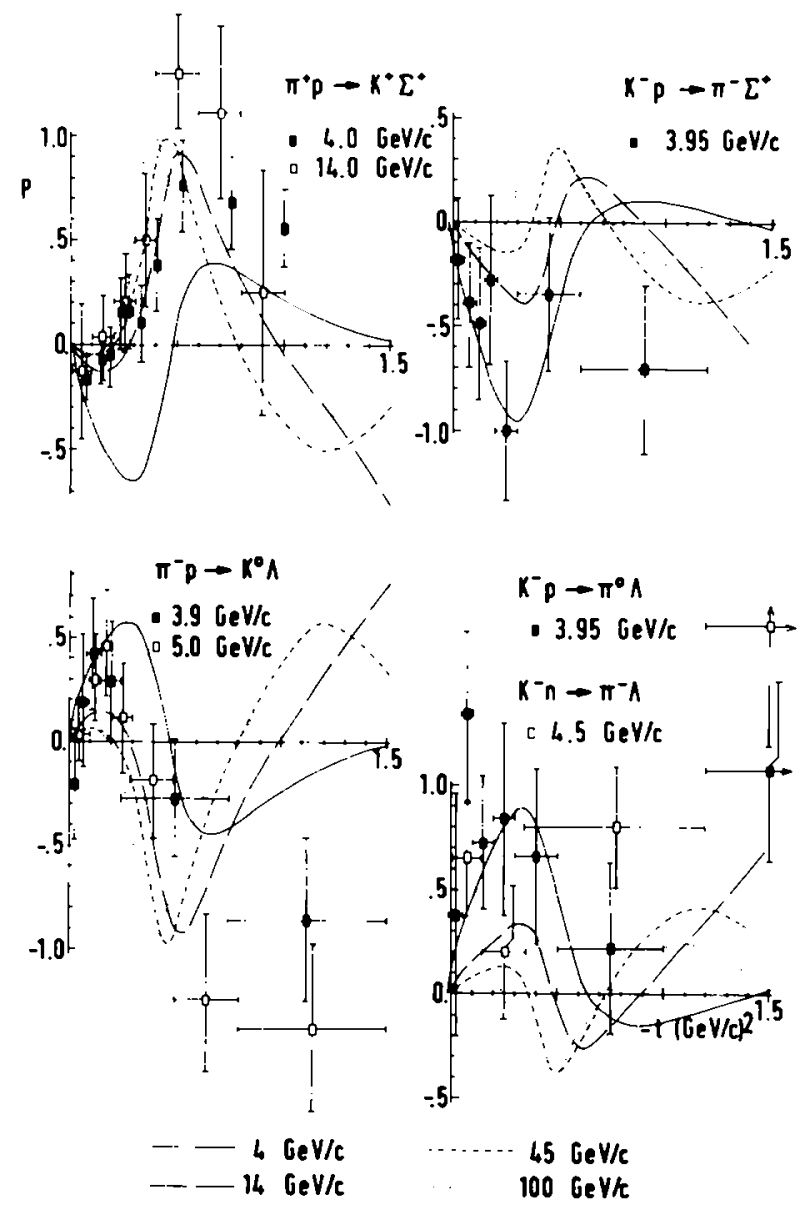

Fig. 28. Global fit polarizations for the hypercharge-exchange reactions. Data from refs. $[47,49$, $50,52,54]$.

Altogether, the phases of our amplitudes seem able to describe polarization effects rather well.

The $\mathrm{KN}, \overline{\mathrm{K}} \mathrm{N}$ and line-reversed reactions. In this section we discuss our view of line reversed reactions. Our approach and our results are different from the usual ones. We will use as examples and emphasize the pair $K^{-} p \rightarrow \bar{K}^{\circ} n$ and $K^{+} n \rightarrow K^{\circ} p$, but the behavior in general and the results also apply to the line-reversed pairs of hyperchargeexchange reactions such as $\pi^{+} p \rightarrow K^{+} \Sigma^{+}$and $K^{--} p \rightarrow \pi^{-} \Sigma^{+}, \pi^{-} p \rightarrow K^{\circ} \Lambda$ and $\mathrm{K}^{-} \mathrm{p} \rightarrow \pi^{\circ} \Lambda$, etc.

Consider the $\rho$ and $A_{2}$ poles. Since $\alpha_{\rho}(0) \approx 0.45$ while $\alpha_{A_{2}}(0) \approx 0.3$ (the exact numbers do not matter as long as $\left.\alpha_{\mathrm{T}}(0)<\alpha_{\mathrm{V}}(0)\right)$ the $\rho\left(\sim i \mathrm{e}^{-\frac{1}{2} i \pi \alpha}\right)$ lies at about $45^{\circ}$ 

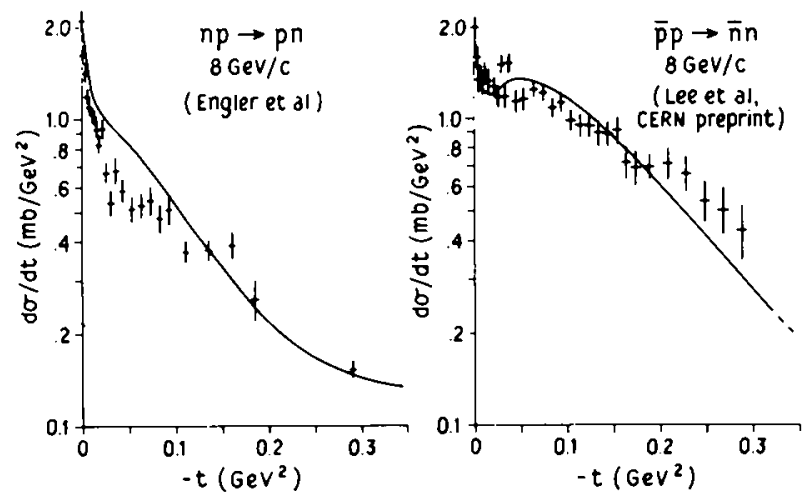

Fig. 29. Preliminary results from ref. $|18|$ for the NN charge-exchange reactions, to illustrate the general applicability of the model to cross sections, polarizations, and amplitudes besides the $0^{-\frac{1}{2}}{ }^{+}$ones. See discussion in text. The $\pi$ is a standard Regge pole as described in the text with $\alpha^{\prime}=1 \mathrm{GeV}^{2}$ and $s_{0}=\frac{1}{2} \mathrm{GeV}^{2}$ and the $\rho$ and $A_{2}$ are the same as in the $0^{-} \frac{1}{2}^{+}$analysis.

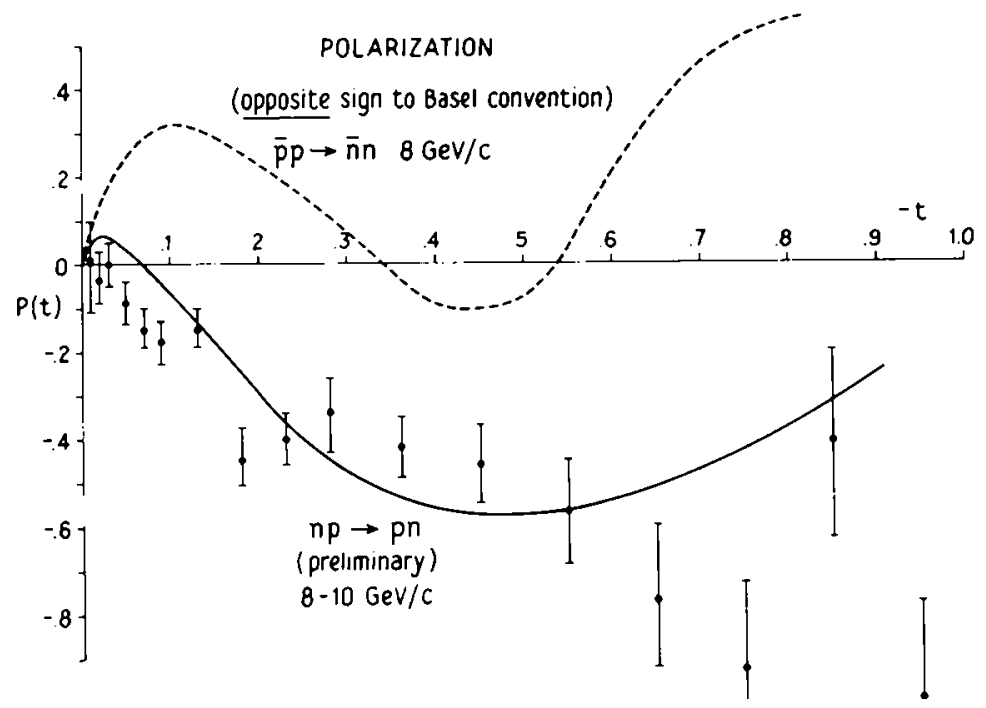

Fig. 30. Preliminary results from ref. $[18]$ for the NN charge-exchange reactions, to illustrate the general applicability of the model to cross sections, polarizations, and amplitudes besides the $0^{-1^{+}}$ones. Sec discussion in text. The $\pi$ is a standard Regge pole as described in the text with $\alpha^{\prime}=1 \mathrm{GeV}^{-2}$ and $s_{0}=\frac{1}{2} \mathrm{GeV}^{2}$ and the $\rho$ and $A_{2}$ are the same as in the $0^{-\frac{1}{2}}$ analysis.

in an Argand plot (say for typical $t$-values in the range $0.05 \lesssim_{-t} \lesssim_{0.35}$ ) while the $\mathrm{A}_{2}\left(\sim \mathrm{e}^{-\frac{1}{2} i \pi \alpha}\right)$ lies at about $150^{\circ}$, more real than imaginary.

For $K^{+} n \rightarrow K^{\circ} p$ or $K^{-} p \rightarrow \bar{K}^{\circ} n$ one has respectively $\rho-A_{2}$ and $\rho+A_{2}$. Since the 


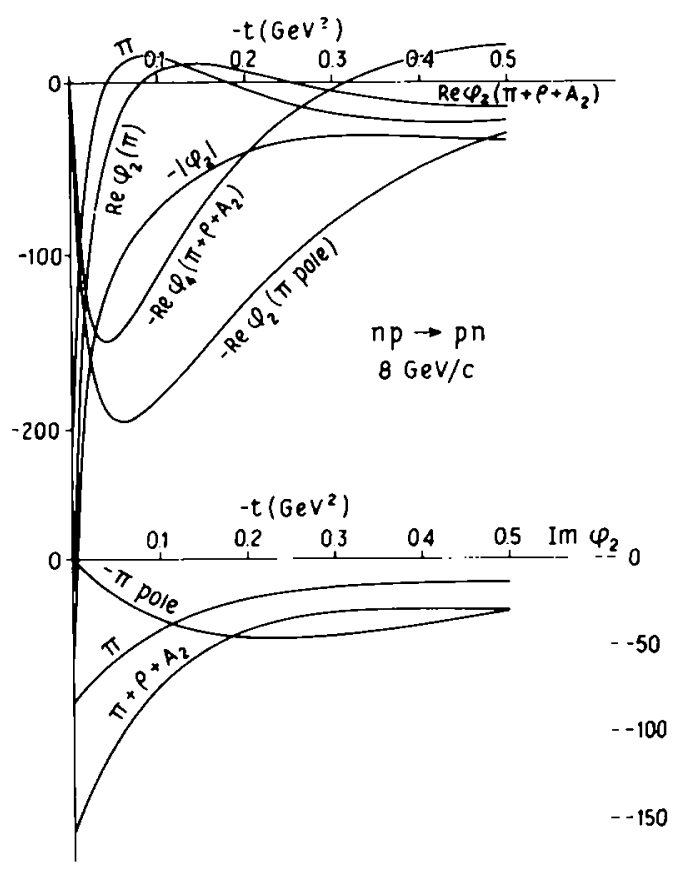

Fig. 31. Preliminary results from ref. [18] for the NN charge-exchange reactions, to illustrate the general applicability of the model to cross sections, polarizations, and amplitudes besides the $0^{-1+}$ ones. See discussion in text. The $\pi$ is a standard Regge pole as described in the text with $\alpha^{\prime}=1 \mathrm{GeV}^{-2}$ and $s_{0}=\frac{1}{2} \mathrm{GeV}^{2}$ and the $\rho$ and $\mathrm{A}_{2}$ are the same as in the $0^{-1^{+}}$analysis. This illustrates for the main new amplitude, the $n=0, x=2$ one (evasive), how the effects arise in detail. Part (a) shows the (dominant) real part of $\varphi_{2}$ for the $\pi$-pole $\left(\sim t /\left(t-m_{\pi}^{2}\right)\right)$, the full $\pi$-amplitude after absorption, the full amplitude including $\rho$ and $A_{2}$. It also includes the magnitude of $\varphi_{2}$, and $\operatorname{Re} \varphi_{4}$. The latter illustrates how $\varphi_{4}$ is suppressed relative to $\varphi_{2}$ because the $\pi$ and $A_{2}$ interfere destructively in $\varphi_{4}$ and constructively in $\varphi_{2}$; this leads to no secondary maximum near $-t \sim 0.05 \mathrm{GeV}^{2}$ in $\mathrm{np} \rightarrow \mathrm{pn}$. Part (b) shows the $\operatorname{lm} \varphi_{2}$, which illustrates the effect of the phase of the pomeron on absorption.

real parts add for $\mathrm{K}^{+}$and cancel for $\mathrm{K}^{-}$we find in general that the magnitude of the pole term is larger for the real process than for the rotating one. This is a qualitative feature of our pole terms and is not sensitive to parameter values.

Next consider how they are absorbed. As is familiar, because $\mathrm{K}^{+}$is more real while $\mathrm{K}^{-}$is more rotating (due here to $\mathrm{A}_{2}$ exchange being less peripheral than $\rho$ ) the former is more strongly absorbed. Thus the $\mathrm{K}^{+}$is absorbed in size down toward $\mathrm{K}^{-}$. In our results the two become approximately equal in the final amplitudes (after absorption) at $P_{\mathrm{L}} \sim 5 \mathrm{GeV} / \mathrm{c}$. As we have scen above, the effective strength of the absorption decreases (due to the shrinkage of the edge contribution to the pomeron) as the energy increases. Consequently, beyond $6-8 \mathrm{GeV} / c$ the $\mathrm{K}^{+}$reaction is no longer absorbed down to the $\mathrm{K}$ reaction and we predict that 


$$
\sigma\left(K^{+} n \rightarrow K^{o} p\right) / \sigma\left(K^{\cdots} p \rightarrow K^{o} n\right)>1,
$$

and increasingly so as $P_{\mathrm{L}}$ increases (until of course at very high energies the $\rho$ begins to dominate both and the ratio goes back to 1). The size of the effect is somewhat model dependent but it is not small - one result is shown in fig. 23 , giving a ratio of about 1.5 at around $30 \mathrm{GeV} / c$. The result holds equally well for the integrated cross section or for $\mathrm{d} \sigma / \mathrm{d} t$ over a range of small- $t$ values (say, $-t \leqslant 0.4$ ). A similar analysis holds for hypercharge-exchange reactions, with similar predictions. For the hypercharge case additional uncertainties such as the relative strengths of absorption and $\mathrm{R}^{*} \mathrm{R}$ cuts make the detailed results even more model dependent, but the basic structure is the same - in all cases the real process will have a bigger cross section than the rotating one even at higher energies. In addition, for all line reversed pairs, the real process is absorbed more so it will have a steeper slope (measured, say, from $0.15 \leqslant \ldots t \leqslant 0.4$ ) than the rotating one.

It is instructive to note what happens as $P_{\mathrm{L}}$ decreases below $5 \mathrm{GeV} / \mathrm{c}$. In our model the absorption strength continues to increase with decreasing $s$, so $\mathrm{K}^{+}$is absorbed more and $\sigma\left(\mathrm{K}^{+}\right)$falls below $\sigma\left(\mathrm{K}^{*}\right)$, contrary to the lower-energy data. Such low-energy disagreement will of ten happen in our model, which is a high-energy one not meant to apply at lower $s$, because as the energy decreases the slope of the Regge pole $\left(\alpha^{\prime} \ln s\right)$ decreases so the pole does not fall off in a reasonable way at larger angles; then the cut formed by integration over the pole gets too big. It is clear in general that a real theory will produce a cut which decreases in size again as one goes toward threshold. Thus in such a theory one will get a result below about $5 \mathrm{GeV} / c$ perhaps like that shown by the dashed line of fig. 23 , and one will predict $\sigma\left(\mathrm{K}^{+} \mathrm{n} \rightarrow \mathrm{K}^{\mathrm{o}} \mathrm{p}\right) / \sigma\left(\mathrm{K}^{\mathrm{p}} \mathrm{p} \rightarrow \mathrm{K}^{\mathrm{o}} \mathrm{n}\right) \geqslant 1$ for all energies.

For the kaon reaction it is currently thought that the cross section ratio is about 1 at both 5 and $12 \mathrm{GeV} / c$. If that persists as experimental normalizations change, our curve (if basically correct) is rising too rapidly at sr.all $s$ because of the low-energy behavior of the model. In any case, we predict $\sigma\left(K^{+} n \rightarrow K^{o} p\right) / \sigma\left(K^{-} p \rightarrow \bar{K}^{o} n\right)>1$ above $12 \mathrm{GeV} / c$, al though we could overestimate the size of the effect, which is very sensitive to details of the interference. A reasonable conjecture for a realistic result is shown by the dashed line in fig. 23 .

Hypercharge-exchange reactions. Considerable work has been devoted to studying hypercharge-exchange processes (HCEX), particularly line-reversed pairs. Their interest is enhanced by the disagreement of simple exchange degeneracy predictions with the data. Since we do not have exchange degenerate poles we view these processes as any other ones. As shown in figures and discussed in the previous section, we do find the correct systematics for cross sections and polarizations, and the $\mathrm{K}^{*}, \mathrm{~K}^{* *}$ amplitudes (for comparison see ref. [33]) are shown in fig. 25.

It appears to us that there is much disagreement about normalizations among the data, and possibly systematic errors in the data. The results are very sensitive to SU(3) quantities and SU(3) breaking, both of which are physically separate from the structure of our model which deals with $s$ - and $t$-dependence of amplitudes. 
Thus it seems to us that an unprejudiced detailed comparison of experiment and theory is not currently possible. Consequently, we simply calculated for the global fit the behavior we expected using the non-HCEX reactions and SU(3) and lowering the $\mathrm{K}^{*}, \mathrm{~K}^{* *}$ intercepts by a (fitted) amount 0.08 from the $\rho, \mathrm{A}_{2}$ intercepts. We found results consistent with the data (for at least one set of reported normalizations). The interested reader can begin with our amplitudes and quickly get results which conform to his prejudices with small changes in the quantities we have fixed or fitted, all given in table 2 .

We obtain results consistent with the data for two main reasons. First, the trajectories are split $\left(\alpha_{\mathrm{A}_{2}}(0)<\alpha_{\rho}(0)\right)$ by an amount $\Delta \alpha \sim 0.1-0.2$, and the $\mathrm{A}_{2}$ is shorter range, which implies the real pole is larger than the rotating one. Second, the phase is rotated by the absorption; the result is sensitive to $\operatorname{Re} M_{\mathrm{cff}}$. Since the absorption strength decreases with energy we expect the real cross section to be larger than the rotating one at almost all energies. See the discussion in the previous section and fig. 24. The polarization systematics can be most easily understood from the amplitudes in fig. 25; the tensor amplitudes are like the vector ones with real and imaginary parts interchanged and with zeros moved out in $t$ because of their shorter range.

If the real member of a pair of line-reversed reactions has a cross section considerably larger $(\sim 30 \%$ ) than the rotating one at $20-40 \mathrm{GeV} / \mathrm{c}$ energies it should be very effective in judging the validity of current ideas. Models with exchange degenerate poles will not easily produce such behavior.

The np and $\overline{\mathrm{p}} \mathrm{p}$ charge exchange. As we remarked above, for completeness we include here a brief analysis of these reactions. The results here are largely due to Vaughn and will be published [18] in more detail as part of a complete study of $\mathrm{NN}$ reactions.

There are several reasons to include these reactions:

(a) They have traditionally been the most difficult processes for high-energy models to treat. One reason for this has been the $\pi$-exchange, which has been a failure for all models except strong absorption ones, and even there it has been a problem at the detailed quantitative level. A second reason is the measured polarization in $n p \rightarrow p n$ which has previously not been understandable in any model. Since we claim a generally applicable model it is important for us to show that we have solved these problems.

(b) Most two-body reactions can be described in terms of four different $s$-channel helicity amplitudes. Two of these, with net helicity flip $n=0$ and $n=1$, occur for the $0^{-} \frac{1}{2}^{+}$reactions. The other two, one with $n=2, x=0$ and the other with $n=0$, $x=2$ (the evasive $n=0$ amplitude) occur in $\mathrm{np} \rightarrow \mathrm{pn}$ and $\mathrm{pp} \rightarrow \mathrm{n} n$. With these amplitudes understood we can go on to describe most other processes without needing new results. In particular, the evasive $n=0$ amplitude is the one responsible for the famous sharp peak of width $m_{\pi}^{2}$ in $n p \rightarrow p n, \gamma p \rightarrow n \pi^{+}, \pi N \rightarrow \rho N$.

(c) An important aspect of our model, which is well illustrated here, is that in a given process all exchanges are absorbed alike: the properties of the absorption are 
assumed to depend only on the external particles and not on the exchange. Older absorption model approaches simply enhanced the absorption strength by a multiplicative factor, $\lambda$, different for each exchange and usually very large for the $\pi$. We do not do that, nor do we need to; the $S_{\text {eff }}$ describing the absorption is the same one for all reggeons $\left(\pi, \rho, \omega, \mathbf{A}_{2}, f\right)$ exchanged in any nucleon-nucleon process, whether elastic or charge exchange.

Complete results on all NN reactions will be published in a paper in preparation [18]. Here we show only the $8 \mathrm{GeV} / c$ charge exchange processes, for the above reasons.

The only subtlety is the $\pi-A_{2}$ interference, which is crucial for quantitative agreement. In the real parts of $\varphi_{2}(n=0, x=2)$ and $\varphi_{4}(n=2, x=0)$ the $\pi$ and $A_{2}$ interfere in opposite ways because the $\pi$ has unnatural parity and the $A_{2}$ natural parity, and $\varphi_{2}$ and $\varphi_{4}$ differ only by putting helicities into minus themselves at one vertex which gives $\varphi_{2}(\pi)=\varphi_{4}(\pi), \varphi_{2}\left(A_{2}\right)=-\varphi_{4}\left(A_{2}\right)$ for the definite parity poles. The interference is constructive in $\varphi_{2}$, destructive in $\varphi_{4}$, so the forward narrow peak is enhanced while the maximum in $\varphi_{4}$ is decreased. The phases arising from absorption with our rotated pomeron again enhance the effect in the direction needed to get quantitative agrecment with data.

Fig. 31 shows the most interesting amplitude, $\varphi_{2}(n=0, x=2)$. The pion pole vanishes at $t=0$ like $t /\left(m_{\pi}^{2}-t\right)$, while the cut does not have to vanish since $n=0$, and varies very slowly with $t$ at small $t$. The $A_{2}$ pole also vanishes at $t=0$ since $x=2$, but without the nearby pole of the $\pi$-case its maximum is further out in $\cdot t$. The $A_{2}$ cut varies slowly in $t$. Because of the different behavior of $\pi$ and $A_{2}$ poles the $\pi+A_{2}$ poic term has a broad maximum, while the $\pi+A_{2}$ cut is still very slowly varying. Thus $\operatorname{Re} \varphi_{2}$ (the main contribution since the $\pi$-pole is mainly real) has a large forward peak and then a broad minimum, independent of the numerical details of the parameters. Since the $\pi$ and $\mathrm{A}_{2}$ occur together in just the same way in $\gamma p \rightarrow \pi^{+} n, \pi N \rightarrow \rho N, K N \rightarrow K^{*} N$, etc., one will find the same effect in all these reactions to the extent the ratio of $\pi$ to $\mathrm{A}_{2}$ couplings is approximately constant for these reactions and for $n p \rightarrow p n$. A similar analysis has already been carried out by Worden [27] for photoproduction reactions, in which he uses the same absorption for all exchanges and verifies that the relative $\pi-\mathrm{A}_{2}$ sign needed can be obtained from symmetry arguments.

Comments. Several points should be emphasized or remarked on.

(i) To avoid any confusion we repeat that the old approximation of the strong absorption model where $M_{\text {eff }}=\lambda M^{\mathrm{el}}$ is not used here. We $d$ o include a contribution from non-elastic intermediate states for the same reasons [4] as previously. In a sense it can be considered as enhancing the edge in impact parameter rather than the whole hadron, with $M_{\text {eff }}=\mathrm{P}+\mathrm{D}$. The pomeron (P) has a central and an edge part while the diffractive inelastic part (D), which is assumed to approximate the contribution of non-clastic intermediate states, arises wholly from the edge and enhances the edge contribution of the pomeron. Thus the one parameter measuring the strength of $\mathrm{D}$ replaces the many $\lambda$ 's of past work; there is a large reduction in the freedom due to parameters and the physical interpretation is improved. 
This distinction is particularly important in $\mathrm{np} \rightarrow \mathrm{pn}$ where it is a qualitative departure from previous work. Now all exchanges including the $\pi$ are absorbed with exactly the same $M_{\text {eff }}$ and the full $\pi$-exchange peak is produced without any extra absorption.

(ii) Our input Regge poles factorize although our full absorbed amplitudes do not. The pole factorization allows us to define coupling constants which can be used wherever an exchange appears. Although we have not had time to check all processes, it appears that our work and previous absorption models suggest that most or perhaps all high-lying exchanges $\left(\rho, \mathrm{A}_{2}, \mathrm{f}, \omega, \pi, \mathrm{N}, \Delta\right)$ can be described in terms of couplings which conform to our prejudices on the values they should have at the particle poles.

(iii) One of the most important virtues of our approach is that it is of general applicability. All other models we are aware of are only formulated for some limited set of reactions or exchanges or amplitudes.

(iv) When making predictions or fits with our model one should proceed as follows. For exchanges used in some previous analysis with our model use trajectories and scalc factors as previously determined. Use an $S_{\text {eff }}$ consistent with a previous one for $\mathrm{KN}, \pi \mathrm{N}, \mathrm{NN}$ reactions, etc. In the pomeron the parameters must have values not very different from those we have specified. With these constraints, calculate what is expected for the reaction in question. By making small changes in various quantitics (c.g., $\alpha_{0}, R_{\mathrm{o}}, \ldots$ ) - perhaps up to $10 \%$ - try to fit existing data for the reaction in question, or related data. Then make a prediction. Our results depend in an essential way on the form of the Regge pole, the pomeron structure, the general structure of $S_{\mathrm{eff}}$, and the split of vector and tensor trajectories and amplitudes.

(v) Note that the absorption correction will always be destructively interfering with the reggeon at small $t$, as it must if it is in fact an absorption correction. When looked at in the complex angular momentum plane, the absorption correction behaves like a Regge cut, usually referred to as Regge-pomeron cut. Since there has been some recent confusion about this, we emphasize here that the sign of this cut is negative with respect to the pole, if the cut arises from absorption. If the amplitude had cuts with positive signs relative to the pole they would not have any thing to do with absorption corrections. Although this contribution contains a cut, we will continue to refer to it as an absorption correction to avoid any confusion of this sort in this paper where we deal mainly with phenomenological applications. The $J$-plane structure of this model will be considered in a different context.

(vi) It may be useful to emphasize the differences between our results and conventional EXD pole models. We have (a) no overall signature zeros in our poles, (b) poles which remember the range of the force by avoiding unphysical states and having $J \sim m^{2}$, (c) splitting of the V-and T-trajectories, and (d) rotated absorption. All of these are essential to obtain our results, which appear to look so like the exchange degenerate pole results and in addition are consistent with experimental data.

A related point is that we are predicting that a given kind of amplitude ( $n, x$ fixed) will behave similarly in all reactions. That will not in general happen in EXD pole models. 
(vii) Because the $A_{2}$ exchange is rather short range the $\pi^{-} p \rightarrow \eta n$ analysis is very sensitive to details of the absorption of the low partial waves and to changes in the low partial waves of the pole. As shown in figs. 18 and 19 the basic behavior we obtain are about right, with a dip at $-t \geq 1 \mathrm{GeV}^{2}$ even though we have no input signature zeros, and a phase behavior suggestive of exchange-degenerate amplitudes. With small changes the interested reader can get a good description of the data.

(viii) Because of the finite nature of computing facilities and our strength we did not ever include the isoscalar-exchange helicity-flip amplitudes. There is no gross behavior of the data which requires them, but they do effect small improvements in the data fit when included. They are essentially uncoupled from the rest of the analysis, only entering into final comparison with a few experimental quantities. By not including them we do not wish to imply that they are not present (for $\omega$ or for even pomeron) but only that their effect is currently only observable at a very detailed, quantitative level.

(ix) We have not considered larger-t behavior. After this work was largely finished, and stimulated by the recent ISR data of Rubbia et al. showing a dip at $-t \sim 1.4 \mathrm{GeV}^{2}$ a better form for the pomeron amplitude has suggested itself. Above and in ref. [6] we assumed that the central part had a Gaussian fall-off in impact parameter. For very strongly interacting hadrons perhaps a better assumption is a sharper fall-off, more like a central black disc in impact parameter. Thus the first term in eq. (3) should be $-i s A \mathrm{e}^{\beta t} J_{1}\left(R_{\mathrm{c}} \sqrt{-t}\right) / R_{\mathrm{c}} \sqrt{t}$.

Here, $R_{\mathrm{c}}$ is the central radius, perhaps of order $\frac{2}{3}-\frac{3}{4} \mathrm{fm}$. Whether $R_{\mathrm{c}}$ should grow with energy as $\sqrt{\ln s}$ or be fixed is not clear; it depends on arguments about the energy dependence of the part of the multiplicity arising from central collisions and on $s$-channel unitarity questions. In either case one gets a good description of the ISR data $0 \leqslant-t \sim 3 \mathrm{GeV}^{2}$; the first zero of $J_{1}$ is at $-t \sim 1.3 \mathrm{GeV}^{2}$ and so is the second zero of $J_{0}$, producing a dip there. The role of the pomeron in absorption is not affected much since that is mainly determined by its small $t$-properties, which are not changed much. The improved form is being fully studied in the nucleon-nucleon analysis of ref. [18], both in its effect on absorption and for elastic scattering where considerable large angle data are available over a range of energies. The systematics as one goes from reaction to reaction are very interesting and will be considered elsewhere.

Arbitrary two-body reactions and exchanges. If it should be true that a given kind of s-channel helicity amplitude for a given exchange behaves the same way in whatever reaction it appears, then it is rather likely that our model will be widely applicable. For data for -- $t \lesssim 1 \mathrm{GeV}^{2}$ many two-body reactions can be described essentially completely in terms of amplitudes we have discussed here (the main exceptions are $n=3$ amplitudes which have a factor $-t^{\frac{3}{2}}$ and are usually small effects for the forward peak). These include vector and tensor exchange for $n=0, x=0 ; n=0, x=2$; $n=1, x=0 ; n=2, x=0$; and $\pi$-exchange for $n=0, x=2$ and $n=2, x=0$. For example, in backward $\pi^{--} \mathrm{p} \rightarrow \mathrm{p} \pi^{-}$the $\Delta$-exchange will behave as forward $\mathrm{A}_{2}$ exchange in the two amplitudes $n=0,1$ and $x=0$, apart from lowering the intercept a little 
Table 1

SU (3) couplings

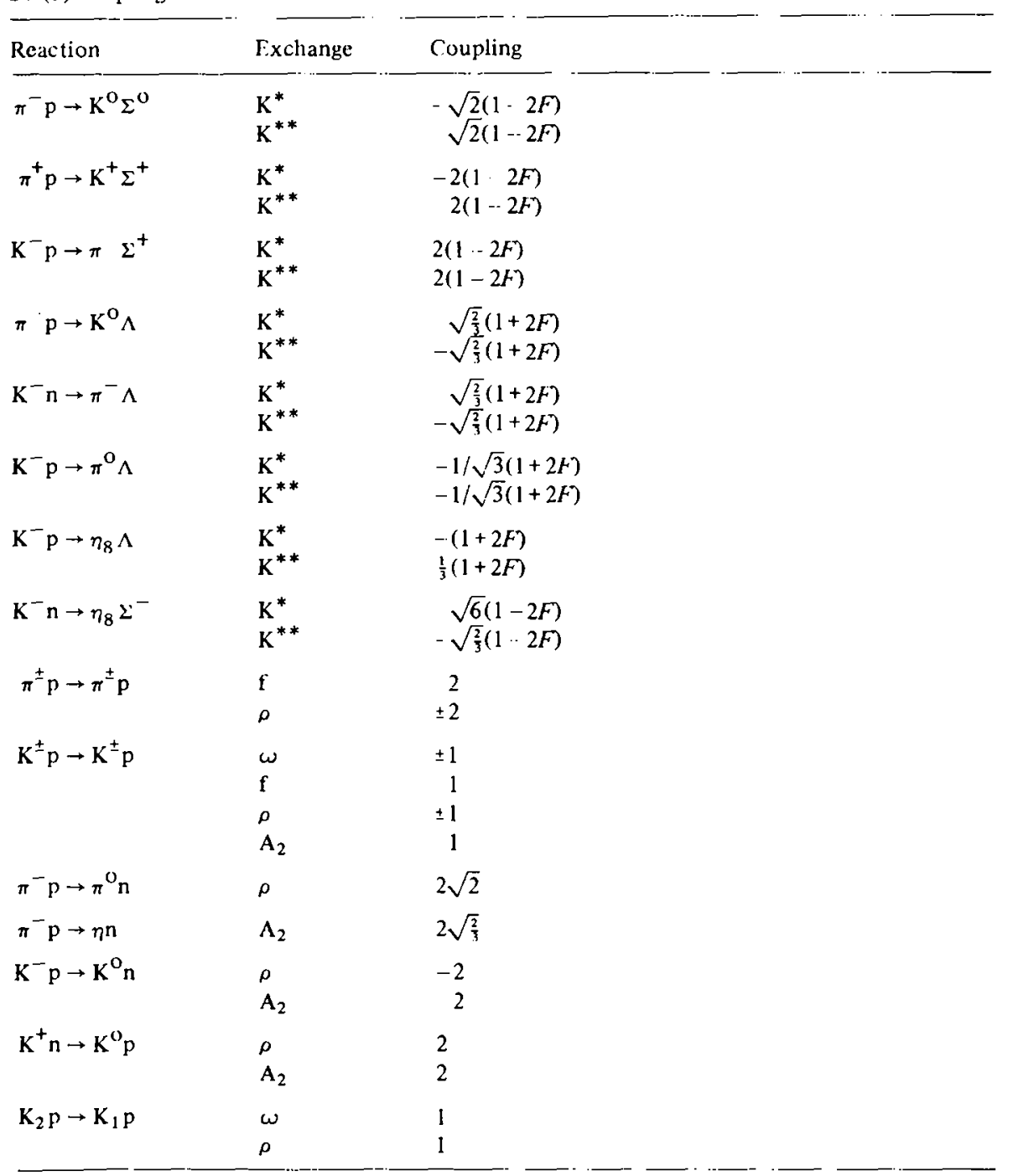

The residues $\gamma_{\mathrm{ca}} \gamma_{\mathrm{db}}$ in the reggeon are calculated by multiplying the SU(3) numbers of table 1 by the $\beta$ 's of table 2 .

and from effects due to McDowell symmetry questions; unfortunately, the latter effects can play an important role in modifying the $t$-dependence of the amplitudes so that calculations will still be necessary.

Thus for his own reactions the reader can make predictions by listing the inde- 


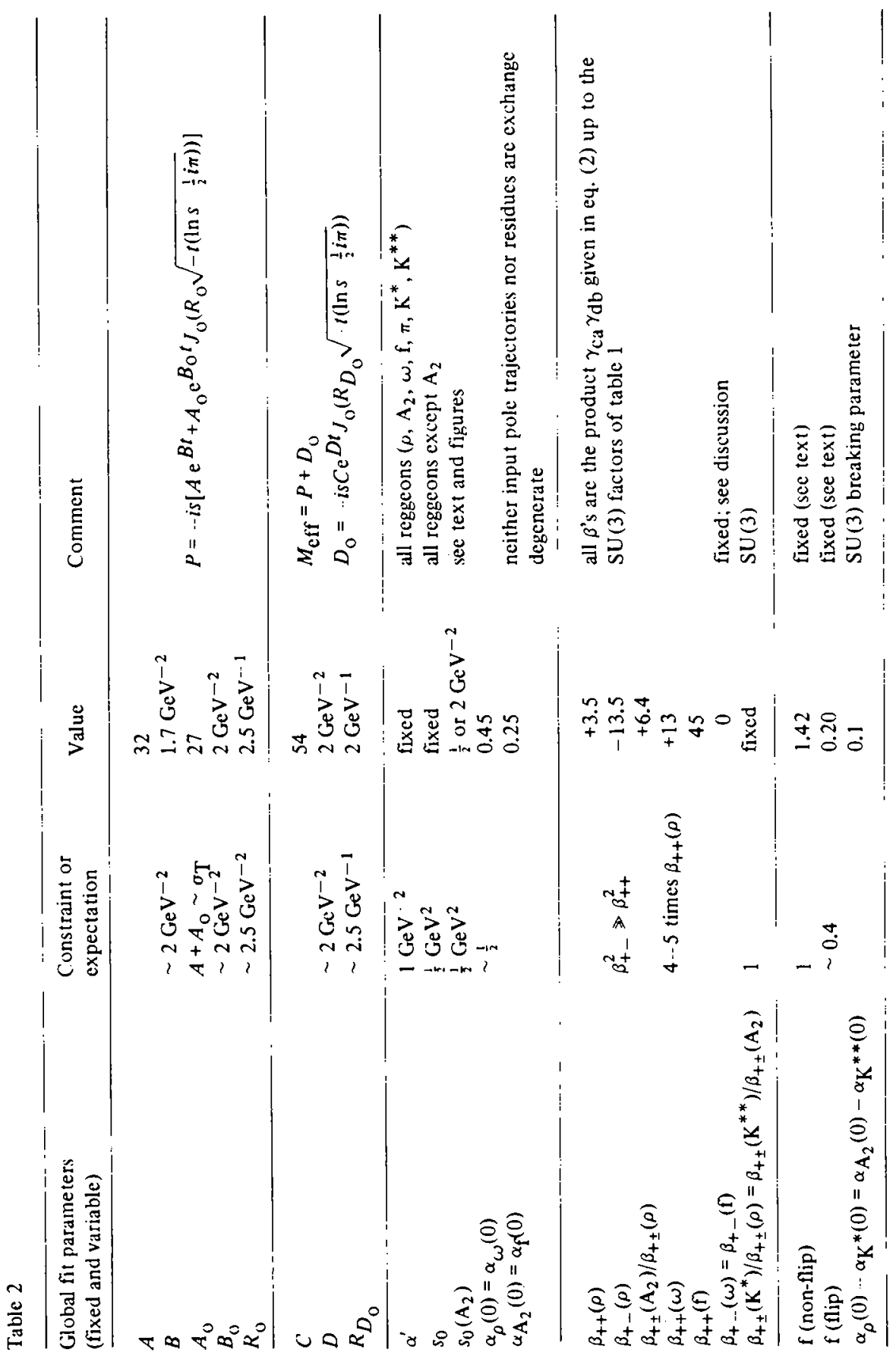


pendent helicity amplitudes involved, writing down the pole term(s) for each amplitude by adding together a term of the form of eq. (2) for each exchange that contributes to that amplitude, taking $S_{\text {eff }}$ from eq. (9) and table 2, and absorbing according the eq. (5) to obtain the full amplitude. The pole residue $\gamma_{\mathrm{ca}} \gamma_{\mathrm{bd}}$ is obtained by multiplying an $S U(3)$ factor from table 1 (defined to be unity for $K N \rightarrow K N$ ) with a factor $\beta$ obtained from table 2 . In appendix 2 we summarize a few details concern. ing the absorption procedure for those who are interested in extensive calculations or minimization. The graphs of our various amplitudes can be used for debugging purposes.

Although the absorption procedure can be carried out analytically with our pomeron if the pole is given an exponential residue, as shown above, for numerical work it is generally just as easy to carry out the actual partial wave expansions or impact parameter transforms numerically. The integrands are never very complicated and no subtleties are encountered apart from computing time questions, discussed in appendix 2. For $\pi$-exchange some care is necessary because of the long tail in impact parameter.

Possible weaknesses. What failures might our model have? It must be tested against other reactions, particularly photoproduction and backward scattering. It is only a high-energy model, with no physics designed to make it applicable in the resonance region; the cuts get too large at very low energies.

At higher energies we are only aware of onc potential shortcoming - we may be absorbing the real parts of tensor-cxchange amplitudes too much. There are three possible clues to this. First, the global fit to $\pi^{-} p \rightarrow \eta n$ is not great, because the real part of the flip amplitude is absorbed a bit too much and has a zero near $-t=1.1$ instead of $-t=1.35$. Second the real part of the f-exchange is too small at $t=0$, giving real parts for $\pi^{ \pm} p$ which are smaller than the data. Third, the difference between line-reversed hypercharge-exchange reactions would be described better if the real part of the $\mathrm{K}^{* *}$ non-flip were absorbed a little less. A related point is that a recent study [29] of the $0^{-} \frac{1}{2}^{+}$reactions uses amplitudes similar to ours for the vector exchange but different ones for tensor exchange, with little absorption of tensor exchange, and gets a reasonable description of the data.

On the other hand, as noted in fig. 19, with fairly small changes we get a rather good description of $\pi^{-} p \rightarrow \eta n$. All of these effects are at a detailed level and we do not really think they indicate anything significantly wrong with the structure of the model, but they should be watched. Perhaps they are a clue to an improvement of $S_{\text {eff }}$.

Our judgement is that we are simply seeing the effects of the short range nature of the tensor exchange, which makes it very sensitive to the details of the absorption of the low partial waves. We suspect that a slightly better understanding of $S_{\mathrm{eff}}$ at small impact parameter would eliminate this possible difficulty. The low-energy behavior is the main improvement of interest phenomenologically.

Physics not in the model. As we have said above, we have tried to construct a very simple model from the point of view of the input physics. That way we can see 
the origins of all of its successes and shortcomings. As we have enthusiastically remarked, the oversimplified model works well. Nevertheless, (a) it does not work perfectly, and (b) certain effects must clearly be part of the physics of any complete model - if they are not important we will have to learn why. In this section we discuss those effects which we are aware of and speculate on where they will show up.

(i) The most obvious omission is lower-lying contributions. It would seem fairly remarkable if only the vector and tensor mesons and the $\pi$ could largely account for the forward meson-exchange data, but it seems (from our results) to be reasonable to claim that. Improved data and more detailed phenomenology will presumably show some places where lower-lying mesons contribute, particularly in vector-meson production [27].

In addition, Regge-Regge cuts are probably important in some reactions. Worden's result [12] argues that they will be small in odd signature reactions, but they could be much larger in even signature ones. As we discussed briefly above, it is possible that we are effectively including some lower-lying contributions in our choice of $S_{\mathrm{eff}}$ (and therefore some effective $\mathrm{R}^{*} \mathrm{R}$ cuts). When data are available over a large enough energy range it should be possible to decide phenomenologically whether $M_{\text {eff }^{--}} P$ falls like a power [28] of $s$.

Although it is very nice to describe data only in terms of high-lying, well-known exchanges, it will ultimately be necessary to account for the absence of lower-lying contributions in the data.

(ii) Another obvious effect which must be understood is the $t$-dependence of Regge residues. Even the small-t analysis could be affected by these because of the integration over $t$ to construct the absorption correction; in different words, if reggeons fell off less rapidly in $t$ than we have assumed. say, they would have larger low partial waves and be more affected by absorption. Given the reasonable quality of our description of the data there does not seem to be much point in trying new forms for the residues until one has a good theoretical reason.

(iii) The most interesting question is the effect of $t$-channel unitarity $[15,30]$ on the absorbed amplitude. This may require the discontinuity of the $J$-plane cut to vanish at the $J$-plane branch point and currently for our non-flip amplitudes it does not. Whether the modifications which are needed will affect the value of the cut at finite energies is an open question. Current work [30] suggests the cut term might gain a factor $\sim 1 /(a+b \ln \ln s)^{m}, m \sim 2$, which would provide additional shrinkage and generally be useful.

For the pomeron itself, $t$-channel unitarity could have a large effect also. It is not yet clear how to modify the current form of the pomeron to insure that it does not violate $t$-channel unitarity. The pomeron structure is complicated enough to give rise to some interesting subtleties. For example, the pomeron form we are using has an essential singularity at $J=1$ but the absorbed amplitude at $t=0$ has no $J$-plane essential singularity. It is clear that there is considerable room here for further understanding.

(iv) Several other more detailed sorts of effects should be considered. For exam- 
ple, if the pomeron is not an SU(3) singlet it will lead to different absorption in different hypercharge-exchange reactions. Similarly, it is possible that the absorption could be weaker in $\pi^{-} \mathrm{p} \rightarrow \eta n$ than in $\pi^{--} p \rightarrow \pi^{\circ} \mathrm{n}\left(\sigma_{\mathrm{T}}(\eta n)<\sigma_{\mathrm{T}}(\pi \mathrm{N})\right)$. The effect of this is illustrated in fig. 19. In general, initial and final state absorption can be different. A full understanding and derivation of $S_{\mathrm{eff}}$ will be required to understand these questions.

In another direction, it may be that $P$ has small helicity-flip contributions, and it is very likely that the diffractive contribution to $S_{\text {eff }}$ has significant helicity-flip contributions. The effect of a small piece in $S_{\text {eff }}$ which flips helicities should be studied. Indeed, a virtue of our approach is that one can systematically improve the understanding and structure of $S_{\mathrm{eff}}$ as better theories or data become available.

Other approaches. By studying our amplitudes we can see in some cases why earlier models have succeeded or failed. We use $V(T)$ for vector- (tensor-) meson exchange.

In the strong absorption model [4] (SAM) with a purely imaginary pomeron there were several effects. In that model one simply enhanced the strength of the clastic absorption by a factor $\lambda$. Then one could get good results for $\operatorname{Im}(V)$, which is indeed strongly absorbed, leading to the Bessel-function systematics [4] $M_{n} \sim$ $J_{n}(R \sqrt{-t})$. In the present paper, on the other hand, the extra absorption of the imaginary part largely arises from the presence of $\operatorname{Re} P$, as discussed above. But for $\operatorname{Re} V$ the effect of multiplying by $\lambda$ or the effect of $\operatorname{Re} P$ were opposite at larger $-t$, so the SAM failed to get good results for $\operatorname{Re} V$ (as indicated by $P\left(\pi^{\circ} p \rightarrow \pi^{\circ} n\right)$ ). For $T$ exchange a similar effect occurs with $\operatorname{Re}(\mathrm{T}) \leftrightarrow \operatorname{Im}(\mathrm{T})$; in addition the reduction in peripherality of the $\mathrm{T}$-poles was not present.

The dual absorption model (DAM) of Harari [32] preserved the most successful part of the SAM, the Bessel-function systematics of Im (V). It also retained the SAM Bessel-function systematics for $\operatorname{Im}(\mathrm{T})$; however, although the matter is far from settled conclusively, most workers fecl that $\operatorname{Im}(\mathrm{T})$ does not behave as $J_{\mathrm{o}}(R \sqrt{-t})$ for $n=0$ amplitudes. In any case the present model shows us why $\operatorname{Re}(\mathrm{V}),(\mathrm{T})$ do not behave as in SAM; if the present model turns out to be correct in its predictions for $\operatorname{Im}(\mathrm{T})$ it will have accounted for why only $\operatorname{Im}(\mathrm{V})$ (but not $\operatorname{Re}(\mathrm{V}), \operatorname{Re}(\mathrm{T}), \operatorname{Im}(\mathrm{T})$ ) behaves as in the SAM or the DAM.

Another standard observation for which we can account is that flip amplitudes behave like Regge poles with signature zeros. We start with an input $\rho$-reggeons with $\operatorname{Rc}(\rho) \sim \sin \frac{1}{2} \pi \alpha_{\rho}$. This has a strong linear zero where $\alpha_{\rho}=0$. But the presence of $\operatorname{Re}(P)$ in the absorption, as we showed above, just rotates the $\operatorname{Re}(\rho)$ until it has a double zero (see fig. 5) as in the case of the pole with signature zero (where $(\rho) \sim$ $1 \ldots \cos \pi \alpha)$. The ways in which our full $\operatorname{Re}(\rho)$ amplitude differs significantly from the pole with signature zero are only quantitative details; ours is larger relative to $\operatorname{Im}(\rho)$ at smaller $t$ and ours does not have as large a secondary maximum at $-t \sim 1$.

Indeed, one of our more surprising and remarkable results is the extent to which all of our V, T amplitudes (sce fig. 5) resemble in their phases those expected from an exchange degenerate pole model. We assume neither exchange degenerate trajec- 
tories for $\rho, \mathrm{A}_{2}$ nor exchange degenerate forms for the poles. The $\pi^{-} \mathrm{p} \rightarrow \pi^{\mathrm{o}} \mathrm{n}$, $\pi \mathrm{p} \rightarrow \eta n$ amplitudes show the differences. But the real and rotating amplitudes, $\mathrm{K}^{+} \mathrm{n} \rightarrow \mathrm{K}^{\mathrm{O}} \mathrm{p}$ and $\mathrm{K}^{-} \mathrm{p} \rightarrow \mathrm{K}^{\mathrm{O}} \mathrm{n}$ respectively, show precisely the real and rotating behavior, modified only by the absorptive zeros. $\mathrm{K}^{+} \mathrm{n}$ is essentially real except where the real part has its zeros; these zeros show up in polarizations (in $\mathrm{K}^{+} \mathrm{p}, \mathrm{pp}$ ), so they can be verified; $\mathrm{K}^{-} \mathrm{p} \rightarrow \mathrm{K}^{\circ} \mathrm{n}$ is really rotating. We have $\alpha_{\mathrm{T}}<\alpha_{\mathrm{V}}$ but $\mathrm{T}$-exchange is shorter range so it is absorbed more and $\alpha_{T}^{\text {eff }}$ is closer to $\alpha_{V}^{\text {eff }}$ than $\alpha_{T}$ is to $\alpha_{V}$. At higher energies the exchange degeneracy gets worse as the $\mathrm{T}$-exchange goes away faster. This prediction will be easily tested at NAL and Serpukhov; some cross sections are shown for $\mathrm{KN}$ in fig. 22 and for $\pi^{+} \mathrm{p} \rightarrow \mathrm{K}^{+} \Sigma^{+}$versus $\mathrm{K}^{--} \mathrm{p} \rightarrow \pi \Sigma^{+}$in fig. 25.

It should perhaps be emphasized that we have found results such as $\operatorname{lm} \rho_{++} \sim J_{\mathrm{O}}$ while $\operatorname{Re} \rho_{+ \pm}$has a double zero in an analytic way, without any special treatment or assumptions concerning amplitudes of any net helicity flip or their real or imaginary parts.

\section{Conclusions}

(a) We have a good chance to describe all data on two-body reactions, in the forward peak, at energies above the resonance region, with a simple model rooted in sound physics.

(b) If our model and interpretations are correct much of the behavior of hadron interactions as functions of $s$ and $t$ and spin is dominated by unitarity effects (well approximated at high energies by absorption) and it will be rather hard to untangle underlying exchange structure. For example, we find very nice exchange-degeneracy properties in some situations but our input is not exchange degenerate and our amplitudes are not dual in any conventional sense. If unitarity will be as crucial a part of any hadron theory as we claim, it does not make one optimistic about arriving at such a theory soon. On the other hand, to make up for this price, perhaps our results are good enough so that we can assume that all the fundamental concepts needed to construct a theory of hadron interactions are already available.

One of us (B.J.H.) would like to thank the Royal Society for the award of a European Fellowship and to acknowledge that this work was initiated while he was a Research Assistant at Imperial College, London. We are indebted to Dr. R.J.N. Phillips for the hospitality extended to us by the Rutherford Laboratory where much of this work was carried out. We have profited considerably from conversations with M. Vaughn, F. Henyey, R. Worden and M. Ross. 


\section{Appendix 1}

\section{Conventions}

We carry out our discussion entircly in terms of $s$-channel helicity amplitudes.

Our normalization is such that (for $0^{-\frac{1}{2}^{+}} \rightarrow 0^{-\frac{1}{2}^{+}}$)

$$
\frac{\mathrm{d} \sigma}{\mathrm{d} t}\left[\mathrm{mb} /(\mathrm{GcV} / \mathrm{c})^{2}\right]=\frac{0.389}{64 \pi k^{2} s}\left(\left|M_{++}\right|^{2}+\left|M_{+--}\right|^{2}\right),
$$

where $M_{++}$and $M_{+. .}$are the non-flip and flip amplitudes, respectively, and

$$
\sigma_{\text {total }}[\mathrm{mb}]=\frac{0.389}{2 k \sqrt{s}} M_{\text {elastic }++}(\theta=0)
$$

where $k$ is the magnitude of the initial state c.m. three momentum and $\theta$ is the c.m. scattering angle.

With our conventions the polarization and spin-rotation parameters are

$$
\begin{aligned}
& P=\frac{-2 \operatorname{Im}\left(M_{++} M_{+. .}{ }^{*}\right)}{\left|M_{++}\right|^{2}+\left|M_{+-\cdot}\right|^{2}}, \\
& R=\frac{-\left(\left|M_{++}\right|^{2}-\left|M_{+\cdot}\right|^{2}\right) \cos \theta_{\mathrm{R}}-2 \operatorname{Re}\left(M_{++} M_{+--}^{*}\right) \sin \theta_{\mathrm{R}}}{\left|M_{++}\right|^{2}+\left|M_{+-}\right|^{2}}, \\
& A=\frac{\left(\left|M_{++}\right|^{2}-\left|M_{+-}\right|^{2}\right) \sin \theta_{\mathrm{R}} 2 \operatorname{Re}\left(M_{++} M_{+--}^{*}\right) \cos \theta_{\mathrm{R}}}{\left|M_{++}\right|^{2}+\left|M_{+-}\right|^{2}},
\end{aligned}
$$

where $\theta_{\mathbf{R}}$ is the nuclear recoil angle in the lab frame, with

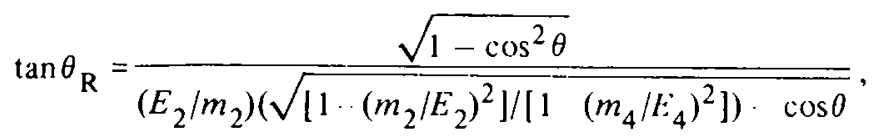

where $m_{2}$ and $E_{2}$ are the mass and c.m. energy, respectively, of the target nucleon, and $m_{4}$ and $E_{4}$ are the mass and c.m. energy of the recoil nucleon, respectively.

\section{Appendix 2}

\section{Numerical procedure}

This section gives a brief outline of our particular method of calculation to help the reader generate his own results without too much difficulty when computing speed is required. 
We used partial wave expansions rather than the impact parameter representation, essentially because we already had an absorption-model program which worked in conjunction with the CERN minimizer MINUIT.

The Regge amplitudes were first expanded in partial wave series

$$
R_{\lambda_{\mathrm{c}} \lambda_{\mathrm{d}} ; \lambda_{\mathrm{a}} \lambda_{\mathrm{b}}}(s, t)=\sum_{j}(2 j+1) R_{\lambda_{\mathrm{c}} \lambda_{\mathrm{d}} ; \lambda_{\mathrm{a}} \lambda_{\mathrm{b}}}^{j}(s) d_{\lambda_{\mathrm{a}}-\lambda_{\mathrm{b}}, \lambda_{\mathrm{c}}-\lambda_{\mathrm{d}}}(\cos \theta)
$$

The partial wave amplitudes, given by

$$
R_{\lambda_{\mathrm{c}} \lambda_{\mathrm{d}} ; \lambda_{\mathrm{a}} \lambda_{\mathrm{b}}}^{j}(s)=\int_{-1}^{+1} \mathrm{~d}(\cos \theta) R_{\lambda_{\mathrm{c}} \lambda_{\mathrm{d}} ; \lambda_{\mathrm{a}} \lambda_{\mathrm{b}}}(s, t) d_{\lambda_{\mathrm{a}}-\lambda_{\mathrm{b}}, \lambda_{\mathrm{c}}-\lambda_{\mathrm{d}}}^{j}(\cos \theta),
$$

were calculated from $j_{\min }=\max \left(\left|\lambda_{\mathrm{a}}-\lambda_{\mathrm{b}}\right|,\left|\lambda_{\mathrm{c}}-\lambda_{\mathrm{d}}\right|\right)$ up to a cut-off $j_{\max }$ (discussed below).

The integrations were performed using an $M$-point gaussian quadrature, according to which

$$
\int_{-1}^{+1} \mathrm{~d} x f(x)=\sum_{m=1}^{M} w_{m} f\left(x_{m}\right)
$$

where $W_{m}$ is the quadrature weight associated with the point $x_{m}$. We define new weights

$$
W_{m}^{\prime}\left(j ; \lambda_{\mathrm{a}} \cdots \lambda_{\mathrm{b}}, \lambda_{\mathrm{c}}, \lambda_{\mathrm{d}}\right)=\frac{1}{2} d_{\lambda_{\mathrm{a}}}^{j} \cdot \lambda_{\mathrm{b}}, \lambda_{\mathrm{c}} \cdots \lambda_{\mathrm{d}}\left(\cos \theta_{m}\right) W_{m}
$$

which were calculated and stored at the beginning of each computer run, thus greatly reduced the running time of the program. This was important since we were minimiz-ng ing, al though it meant storing large arrays. The symmetry propertics

$$
d_{\lambda \mu}^{j}(x)=(-1)^{\lambda-\mu} d_{\mu \lambda}^{j}(x)=d_{-\mu,-\lambda}^{j}(x),
$$

reduced the number of different sets of new weights needed. We then calculated the partial wave amplitudes using the formula

$$
R_{\lambda_{c}, \lambda_{d} ; \lambda_{\mathrm{a}}, \lambda_{\mathrm{b}}}^{i}(s)=\sum_{m=1}^{M} R_{\lambda_{\mathrm{c}} \lambda_{\mathrm{d}} ; \lambda_{\mathrm{a}} \lambda_{\mathrm{b}}}\left(s, \cos \theta_{m}\right) w_{m}^{\prime}\left(j ; \lambda_{\mathrm{a}}-\lambda_{\mathrm{b}}, \lambda_{\mathrm{c}}-\lambda_{\mathrm{d}}\right) .
$$

The absorbed partial wave amplitudes were obtained from the standard absorptionmodel prescription

$$
M_{\lambda_{c}, \lambda_{\mathrm{d}} ; \lambda_{\mathrm{a}}, \lambda_{\mathrm{b}}}^{j}(s)=S_{\mathrm{eff}}^{j} R_{\lambda_{\mathrm{c}}, \lambda_{\mathrm{d}} ; \lambda_{\mathrm{a}}, \lambda_{\mathrm{b}}}^{j}(s)
$$

with $R$ and $S_{\text {eff }}$ as discussed in this paper. 
The absorbed amplitude is given by

$$
M_{\lambda_{c}, \lambda_{d} ; \lambda_{a}, \lambda_{b}}(s, t)=\sum_{j=j_{\min }}^{\infty}(2 j+1) M_{\lambda_{c}, \lambda_{d} ; \lambda_{a}, \lambda_{b}}^{j}(s) d \lambda_{\lambda_{c}-\lambda_{d}, \lambda_{a}-\lambda_{b}}(\cos \theta)
$$

We chose $i_{\max }$ to be at least large enough to give $S_{\text {eff }} j_{\max }=1$, since then partial wave amplitudes with $j>i_{\max }$ are unaltered by the absorption. Hence

$$
\begin{aligned}
& M_{\lambda_{\mathrm{c}}, \lambda_{\mathrm{d}} ; \lambda_{\mathrm{a}}, \lambda_{\mathrm{b}}}(s, t) \\
& =\sum_{j=j_{\min }}^{j_{\max }}(2 j+1)\left[M_{\lambda_{\mathrm{c}}, \lambda_{\mathrm{d}} ; \lambda_{\mathrm{a}}, \lambda_{\mathrm{b}}}^{j}(s)-R_{\lambda_{\mathrm{c}}, \lambda_{\mathrm{d}} ; \lambda_{\mathrm{a}}, \lambda_{\mathrm{b}}}^{j}(s)\right] \\
& \quad \times d_{\lambda_{\mathrm{a}}-\lambda_{\mathrm{b}}, \lambda_{\mathrm{c}}-\lambda_{\mathrm{d}}}^{j}(\cos \theta)+R(s, t) .
\end{aligned}
$$

It was therefore not necessary to continue the partial wave expansion to sufficiently large $j$ for the contribution of the higher partial wave amplitudes to be negligible. This is a particularly important detail for $\pi$-exchange.

The value of $j_{\max }$ increases with increasing energy, and hance the higher the order of Gaussian quadrature required to give the high partial wave amplitudes accurately. Experience showed $j_{\max }=50$ and $M=48$ to be large enough, al though smaller values were sufficient at lower energies.

\section{References}

[1] R.J.N. Phillips, Rapporteur talk at the Amsterdam Int. Conf. on elementary particles, 1971.

[2] C. Michael, Rapporteur talk at the Oxford Int. Conf. on high-energy collisions, April, 1972.

[3] G. Cohen-Tannoudji, A. Morel and Ph. Salin, Nuovo Cimento 55A (1968) 412.

[4] M. Ross, F.S. Henyey and G.L. Kane, Nucl. Phys. B23 (1969) 269.

[5] R. Kelly, C.L. Kane and F.S. Henyey, Phys. Rev. Letters 24 (1970) 1511.

[6] G. L. Kane, Phys. Letters 40B (1972) 363.

[7] L. Stodolsky, SLAB preprint, unpublished.

[8] V. Barger and R.J.N. Phillips, Phys. Rev. 187 (1969) 2210.

[9] R.L. Kelly, Phys. Letters B39 (1972) 635.

[10] G. Höhler, in High-energy phenomenology, Proc. of the Six th Rencontre de Moriond, ed. J. Tran Van.

[11] M.H. Ross, Phys. Letters 38 B (1972) 321.

[12] B.J. Hartley and G.L. Kane, Phys. Letters 39B (1972) 531.

[13] O. Overseth, private communication.

[14] G.L. Kane, Phenomenology of diffractive reactions, Lectures at the XII Crakow School of Theoretical Physics, Zakopane, Poland, June, 1972; Rutherford Lab preprint RRP/T/20.

[15] V.N. Gribov, I.Ya. Pomeranchuk and K.A. Ter Martirosyan, Phys. Rev. 139 (1965) B184.

[16] R. Worden, Phys. Letters 40B (1972) 260. 
[17] G. Fox, Proc. of the Third Int. Conf. on high-energy collisions, Stony Brook, New York, 1969.

[18] M. Vaughn and C.I. Kane, in preparation.

[19] F. Halzen and C. Michael, Phys. Letters 36B (1971) 367.

[20] S.P. Denisov et al., Phys. Letters 36B (1971) 415.

(21) V.N. Bolotovet al., Phys. Letters 38B (1972) 120.

[22] G. Barbeillini et al., Phys. Letters 39B (1972) 663.

[23] P. Bonamy et al., preprint (1972).

[24] D. Hill et al., Paper No. 212 , XVI Int. Conf. on high-energy physics, Chicago, September, 1972.

[25] (IERN-Orsay-Pisa Collaboration, presented by L. Dick at the Second Int. Conf. on polarized targets, Berkeley, 1971.

[26] np $\rightarrow$ pn: Argonne, Ohio State, Michigan State collaboration, submitted to the Oxford Int. Conf. on high-energy collisions, and to be published;

P.R. Robrish et al., Phys. Letters 31 B (1970) 617; $\bar{p} p \rightarrow \bar{n} n$ : CERN, F.TH Zurich, IC London, CIN Saclay Collaboration, submitted to the XVI Int. Conf. on high-energy physics, September, 1972, and to be published.

[27] R. Worden, Nucl. Phys. B37 (1971) 253.

[28] A. Martin and P. Stevens, Phys. Rev. D5 (1972) 147.

[29] G.A. Ringland, R.G. Roberts, D.P. Roy and J. Tran Thanh Van, Nucl. Phys. B44 (1972) 395.

[30] F. Guerin, Nice preprint;

I. Henyey, D. Richards and W. Zakrzewski, to be published.

[31] J. Bronzan and C.E. Jones, Phys. Rev. 160 (1967) 1494.

[32] H. Harari, Phys. Rev. Letters 26 (1971) 1400.

[33] V. Barger and A.D. Martin, Phys. Letters 39B (1972) 379;

J.S. Lous and J.A.J. Matthews, SLAC preprint.

[34] V. Barger, K. Geer and R.J.N. Phillips, Nucl. Phys. B36 (1972).

[35] G.A. Ringland and D.P. Roy, Phys. Letters 39B (1972) 531.

[36] W. Galbraith el al., Phys. Rev. 138 (1965) B913;

W.F. Baker et al., Phys. Rev. 129 (1967) 2285;

K.J. livley et al., Phys. Rev. Letters 19 (1967) 330;

S.P. Denisov et al., Phys. Letters 36B (1971) 415; Phys. Letters 36B (1971) 528.

[37] S. Brandt ct al., Phys. Rev. Letters 10 (1963) 413;

K.J. Foley et al., Phys. Rev. Letters 11 (1963) 425;

C.T. Coffin et al., Phys. Rev. 159 (1967) 1169;

D. R. Rust et al., Phys. Rev. Letters 24 (1970) 1361.

[38] K.J. Foley et al., Phys. Rev. Letters 11 (1963) 503;

W. De Bacre et al., Nuovo Cimento 45A (1966) 885;

J. Mott et al., Phys. Letters 23 (1966) 171;

M. Aderholz et al., Phys. Letters 24 (1967) 434;

R.J. Miller et al., Phys. Letters 34 B (1971) 230.

[39] K.J. Foley et al., Phys. Rev. 181 (1969) 1775.

[40] M. Borghini et al., Phys. Letters 31 B (1970) 405; CFRN preprint (1972).

[41] A. De Lesquen et al., Phys. Letters 40B (1972) 278.

[42] A.V. Stirling et al., Phys. Rev. Letters 14 (1965) 763;

P. Sonderegger et al., Phys. Letters 20 (1966) 75.

[43] O. Guisan et al., Phys. Letters 18 (1965) 200;

K. Ruddick et al., private communication.

[44] D.D. Drobnis et al., Phys. Rev. Letters 20 (1968) 274;

P. Bonamy et al., Nucl. Phys. B16 (1970) 335; ref. [23]. 
[45] P. Astbury et al., Phys. Letters 23 (1966) 396;

D. Cline et al., University of Wisconsin report (1969); Phys. Rev. Letters 23 (1969) 1318;

A. Firestone et al., Phys. Rev. Letters 25 (1970) 958;

M. Haguenauer et al., Phys. Letters 37B (1971) 538.

[46] E.H. Willen et al., paper contributed to Oxford Conf., 1972.

[47] S.M. Pruss et al., Phys. Rev. Letters 23 (1969) 189;

A. Bashian et al., Phys. Rev. D4 (1971) 2667.

[48] P. Kalbaci et al., Phys. Rev. Letters 27 (1971) 74.

[49] C.E.W. Ward et al., Argonne National Laboratory preprint (1971).

[50] L. Moscoso et al., Nucl. Phys. 36B (1972) 332.

[51] D. Birnbaum et al., Phys. Letters 31 B (1970) 484.

[52] W.L. Yen et al., Phys. Rev. 188 (1969) 2011.

[53] D.J. Crennell et al., Phys. Rev. Letters 23 (1969) 1347.

[54] M. Abramovich et al., Nucl. Phys. B27 (1971) 477. 